\title{
Wave propagation modeling in periodic elasto-thermo-diffusive materials via multifield asymptotic homogenization
}

\author{
Francesca Fantoni ${ }^{1, *}$, Andrea Bacigalupo ${ }^{2, *}$ \\ ${ }^{1}$ DICATAM, Università degli Studi di Brescia, via Branze 43, 25123, Brescia, Italy \\ 2 DICCA, Università degli Studi di Genova,via Montallegro 1, 16145 Genova, Italy
}

February 27, 2020

\begin{abstract}
A multifield asymptotic homogenization technique for periodic thermo-diffusive elastic materials is provided in the present study. Field equations for the first-order equivalent medium are derived and overall constitutive tensors are obtained in closed form. These lasts depend upon the micro constitutive properties of the different phases composing the composite material and upon periodic perturbation functions, which allow taking into account the effects of microstructural heterogeneities. Perturbation functions are determined as solutions of recursive non homogeneous cell problems emanated from the substitution of asymptotic expansions of the micro fields in powers of the microstructural characteristic size into local balance equations. Average field equations of infinite order are also provided, whose formal solution can be obtained through asymptotic expansions of the macrofields. With the aim of investigating dispersion properties of waves propagating inside the medium, proper integral transforms are applied to governing field equations of the homogenized medium. A quadratic generalized eigenvalue problem is thus obtained, whose solution characterizes the complex valued frequency band structure of the first-order equivalent material. The validity of the proposed technique has been confirmed by the very good matching obtained between dispersion curves of the homogenized medium and the lowest frequency ones relative to the heterogeneous material. These lasts are computed from the resolution of a quadratic generalized eigenvalue problem over the periodic cell subjected to Floquet-Bloch boundary conditions. An illustrative benchmark is conducted referring to a Solid Oxide Fuel Cell (SOFC)-like material, whose microstructure can be modeled through the spatial tessellation of the domain with a periodic cell subjected to thermo-diffusive phenomena.
\end{abstract}

\section{Introduction}

The increasing need of energy diversification and employment of alternative and renewable energy sources motivates the growth in the use of fuel cells as power generating systems. Substitution of conventional fuel combustion with an electrochemical reaction in order to generate electricity make fuel cells clean and sustainable energy devices, nowadays exploited for a wide range of applications, from powering satellites to generating power for vehicles and buildings. Fuel cells consist of two porous heat resistant electrodes, the negative one (anode) and the positive one (cathode), undergoing electrochemical reaction in order to produce an electric current. They are sandwiched around a porous electrolyte, which is the ion conductor. Fuel cells differ according to the electrolyte employed, which influences the type of occurring electrochemical reaction, of the catalyst, and of the fuel, thus achieving distinct levels of efficiency (Brandon and Brett, 2006). In this context, Solid Oxide Fuel Cells (SOFCs) are characterized by having a doped, solid, ceramic material to form the electrolyte and they excel for their high electrical efficiency and low operating costs (Zhu and Deevi, 2003, Bove and Ubertini, 2008). The cathode of SOFCs is supplied both with oxygen, acting as the oxidant, and electrons coming from the external electrical circuit. Oxygen ions intercalate into the electrolyte as a consequence of the reduction process taking place at the cathode side. Through the solid electrolyte, negative oxygen ions are therefore conducted from the cathode to the anode, where they combine with the

* Corresponding authors: Tel:+39 0303711330,

E-mail addresses: francesca.fantoni@unibs.it; andrea.bacigalupo@unige.it 
hydrogen fuel, thus generating both water and electrons as products of the oxidation reaction. Electrical current is hence generated by electrons travelling along the external circuit and then reentering into the cathode material. In addition, every single cell is characterized by flow channels for air and fuel and by a metallic or ceramic interconnect separator, which allows connecting cells in series with the aim to produce sufficient voltage for the practical use.

Macroscopic engineering response of such multiphase materials is strongly influenced by the mechanics and physics occurring at the microscale, whose characteristic size is very small compared to the structural one. For this reason a numerical analysis of microstructured devices like a SOFCs stack could reveal extremely challenging in terms of computational and temporal resources (Hajimolana et al., 2011, Dev et al. 2014). When scales separation holds, homogenization techniques result to be remarkably useful in order to provide an accurate and concise description of the medium which properly take into account the behavior and the mechanical response of the microstructure. The application of homogenization methods and multiscale modelings allows avoiding the demanding numerical computation of the whole heterogeneous medium leading to the identification of effective macroscopic properties for the equivalent continuum. In order to study the overall properties of composite materials, numerous homogenization approaches have been provided over the last decades, which can be divided in asymptotic techniques (Sanchez-Palencia, 1974, Bensoussan et al., 1978; Bakhvalov and Panasenko, 1984: Gambin and Kröner, 1989; Allaire, 1992; Bacigalupo, 2014, Fantoni et al. 2017, 2018), variational-asymptotic techniques (Smyshlyaev and Cherednichenko, 2000. Peerlings and Fleck, 2004, Bacigalupo and Gambarotta, 2014), and numerous identification approaches including the analytical (Bigoni and Drugan, 2007, Milton and Willis, 2007, Bacca et al., 2013a b c, Nassar et al., 2015: Bacigalupo et al., 2018) and computational methods (Forest and Sab. 1998; Ostoja-Starzewski et al. 1999, Feyel and Chaboche, 2000; Kouznetsova et al., 2002; Forest, 2002, Feyel, 2003; Kouznetsova et al. 2004; Lew et al., 2004; Kaczmarczyk et al., 2008; Yuan et al., 2008; Scarpa et al., 2009; Bacigalupo and Gambarotta, 2010; Forest and Trinh, 2011, De Bellis and Addessi, 2011, Addessi et al., 2013, Zäh and Miehe, 2013 Salvadori et al. , 2014: Trovalusci et al., 2015). The present study is devoted to provide a multifield asymptotic homogenization technique for periodic thermo-diffusive materials considering as periodic cell the typical SOFC building block. An accurate prediction of the overall response of SOFCs is of crucial importance in order to guarantee the satisfaction of design requirements and the reliability of the entire system. Battery devices like SOFCs, in fact, are subjected to severe stresses due to high operating temperatures $\left(600^{\circ}-1000^{\circ}\right)$ (Pitakthapanaphong and Busso, 2005) and intense particle diffusion, which could compromise their efficiency in terms of power generation and energy conversion, ultimately impacting on their failure behavior (Atkinson and Sun, 2007; Kuebler et al., 2010, Delette et al., 2013). Previous numerical models of SOFCs focused on electrochemical aspects can be found in (Kakac et al., 2007, Colpan et al. 2008), while mechanical properties of each phase forming the composite battery device are presented in (Hasanov et al. 2011). Latterly, different multiscale modeling of SOFCs have been provided focusing on computational homogenization (Kim et al., 2009: Muramatsu et al., 2015, Molla et al., 2016), asymptotic first-order homogenization of thermo-mechanical properties (Bacigalupo et al., 2016), and asymptotic non local homogenization of elastic properties (Bacigalupo et al., 2014) where the influence of temperature upon local and non local overall constitutive tensors has been studied. Furthermore, an investigation of the complex frequency band structure of periodic SOFCs based on a micromechanical perspective has been recently presented by one of the author in (Bacigalupo et al. 2019). Nevertheless, to the best of authors' knowledge, a rigorous quantitative multiscale description of mechanical, thermal, and diffusive properties of SOFC-like material and their coupling is still missing. In the followings, down-scaling relations are provided. They relate the microfields, specifically the displacement, the relative temperature and the chemical potential to the macroscopic fields and their gradients by means of perturbation functions. These lasts are regular, periodic functions derived through the resolution of recursive, non homogeneous differential problems, known as cell problems, obtained inserting an asymptotic expansion of the microfields in powers of the microstructural length scale into the local balance equations and reordering at the different orders of the micro characteristic size. Following the rigorous approach described in (Smyshlyaev and Cherednichenko, 2000, Bacigalupo, 2014), average field equations of infinite order are obtained from the substitution of down-scaling relations into micro governing field equations. A formal solution of the average field equations of infinite order can be attained by performing an asymptotic expansion of the macrofields in powers of the micro length scale, and truncation of resulting equations to the zeroth order allows characterizing field equations of the first-order equivalent medium for the class of periodic thermo-diffusive materials considered. Coefficients of obtained field equations are related to the overall constitutive tensors, whose expression is provided in closed form in 
terms of perturbation functions and microscopic constitutive properties.

With the aim of investigating the dispersive free waves propagation within the periodic microstructured material, bilateral Laplace transform in time and Fourier transform in space are applied to field equations of the homogenized medium, thus obtaining a quadratic generalized eigenvalue problem, whose solution characterizes the complex frequency band structure of the first-order equivalent medium. The validity of the proposed approach is assessed by comparing the obtained complex frequency spectra with the ones relative to the heterogeneous thermo-diffusive material. In this case, a generalization of the Floquet-Bloch theory is employed, which allows determining dispersion properties of the heterogeneous material by solving a generalized quadratic eigenvalue problem over the periodic cell endowed with Floquet-Bloch boundary conditions. Finally, an asymptotic approximation of the complex spectrum for the first-order equivalent medium is performed via perturbative technique. This allows achieving a parametric approximation of the complex frequency in powers of the wave vector in terms of the overall constitutive parameters and obtained explicit dispersion curves demonstrate to match very well with the ones relative to the homogenized medium. The work is organized as follows: Section 2 describes the governing microscopic field equations and recursive differential problems obtained through asymptotic expansion of the microfields in powers of the microstructural length scale. Cell problems and relative perturbation functions at the different orders of the micro characteristic size are detailed in Section 3 . Section 4 is devoted to the determination of downscaling and up-scaling relations, while in Section 5 field equations of the first-order equivalent continuum are presented and overall constitutive tensors are provided in closed form. The determination of complex frequency band structure for the first-order homogenized medium is described in Section 6, together with its asymptotic approximation via perturbative method in Section 6.1. In order to evaluate the capabilities of the proposed method a representative example is performed in Section 7, where the complex frequency band structure and its asymptotic approximation are provided for the equivalent continuum in relation to a typical SOFC and obtained results are compared with the ones of the relative heterogeneous periodic cell. Final remarks are then proposed in Section 8

\section{Periodic heterogeneous thermo-diffusive material: field equa- tions and multi-scale description}

Under the assumption of small strains, the heterogeneous microstructured composite material depicted in figure 1 is described as a linear thermo-diffusive Cauchy medium (Nowacki, 1974a b c). In a two-dimensional perspective, as represented in figure 1, vector $\mathbf{x}=x_{1} \mathbf{e}_{1}+x_{2} \mathbf{e}_{2}$ defines the position of each material point in the orthogonal reference system $\left\{O, \mathbf{e}_{1}, \mathbf{e}_{2}\right\}$. Micro fields characterizing the first-order continuum are the displacement field $\mathbf{u}(\mathbf{x}, t)=u_{i}(\mathbf{x}, t) \mathbf{e}_{i}$, relative temperature field $\theta(\mathbf{x}, t)=T(\mathbf{x}, t)-T_{0}$ with $T(\mathbf{x}, t)$ the absolute temperature and $T_{0}$ a reference stress free temperature, and chemical potential field $\eta(\mathbf{x}, t)$. Being $\varepsilon$ the characteristic size of the microstructure, two periodicity vectors $\mathbf{v}_{1}=d_{1} \mathbf{e}_{1}=\varepsilon \mathbf{e}_{1}$ and $\mathbf{v}_{2}=$ $d_{2} \mathbf{e}_{2}=\delta \varepsilon \mathbf{e}_{2}$ identify the periodic cell $\mathcal{A}=[0, \varepsilon] \times[0, \delta \varepsilon]$ (figure 1 1 (b)). Rescaling cell $\mathcal{A}$ by the length $\varepsilon$, the periodic microstructure is obtained by spanning the nondimensional unit cell $\mathcal{Q}=[0,1] \times[0, \delta]$, as depicted in figure 1 1 (c). The separation between the macro and the micro scales is mathematically described by two distinct variables, namely the macroscopic (or slow) one $\mathbf{x} \in \mathcal{A}$ and the microscopic (or fast) one $\boldsymbol{\xi}=\mathbf{x} / \varepsilon \in \mathcal{Q}$ (Bakhvalov and Panasenko, 1984, Smyshlyaev and Cherednichenko, 2000, Peerlings and Fleck, 2004, Bacigalupo, 2014). Micro-stress tensor $\boldsymbol{\sigma}(\mathbf{x}, t)=\sigma_{i j}(\mathbf{x}, t) \mathbf{e}_{i} \otimes \mathbf{e}_{j}$, micro heat flux vector $\mathbf{q}(\mathbf{x}, t)=q_{i}(\mathbf{x}, t) \mathbf{e}_{i}$, and mass flux vector $\mathbf{j}(\mathbf{x}, t)=j_{i}(\mathbf{x}, t) \mathbf{e}_{i}$ are determined by the coupled constitutive relations (Nowacki, 1974a|b c)

$$
\begin{aligned}
\boldsymbol{\sigma}(\mathbf{x}, t) & =\mathfrak{C}^{(m, \varepsilon)} \boldsymbol{\varepsilon}(\mathbf{x}, t)-\boldsymbol{\alpha}^{(m, \varepsilon)} \theta(\mathbf{x}, t)-\boldsymbol{\beta}^{(m, \varepsilon)} \eta(\mathbf{x}, t), \\
\mathbf{q}(\mathbf{x}, t) & =-\boldsymbol{K}^{(m, \varepsilon)} \nabla \theta(\mathbf{x}, t), \\
\mathbf{j}(\mathbf{x}, t) & =-\boldsymbol{D}^{(m, \varepsilon)} \nabla \eta(\mathbf{x}, t),
\end{aligned}
$$

where symbol $\varepsilon(\mathbf{x}, t)=\operatorname{sym} \nabla \mathbf{u}(\mathbf{x}, t)$ is the micro small strains tensor and superscript $m$ refers to the microscale. In equations $1 \mathrm{a})-1 \mathrm{c}) \mathfrak{C}^{(m, \varepsilon)}=C_{i j k l}^{m}\left(\boldsymbol{\xi}=\frac{\mathbf{x}}{\varepsilon}\right) \mathbf{e}_{i} \otimes \mathbf{e}_{j} \otimes \mathbf{e}_{k} \otimes \mathbf{e}_{l}$ is the fourth order micro elasticity tensor having major and minor symmetries, $\boldsymbol{\alpha}^{(m, \varepsilon)}=\alpha_{i j}^{m}\left(\boldsymbol{\xi}=\frac{\mathbf{x}}{\varepsilon}\right) \mathbf{e}_{i} \otimes \mathbf{e}_{j}$ is the symmetric second order micro thermal dilatation tensor, $\boldsymbol{\beta}^{(m, \varepsilon)}=\beta_{i j}^{m}\left(\boldsymbol{\xi}=\frac{\mathbf{x}}{\varepsilon}\right) \mathbf{e}_{i} \otimes \mathbf{e}_{j}$ is the symmetric second order micro diffusive expansion tensor, $\boldsymbol{K}^{(m, \varepsilon)}=K_{i j}^{m}\left(\boldsymbol{\xi}=\frac{\mathbf{x}}{\varepsilon}\right) \mathbf{e}_{i} \otimes \mathbf{e}_{j}$ is the symmetric second order micro heat conduction tensor, and 


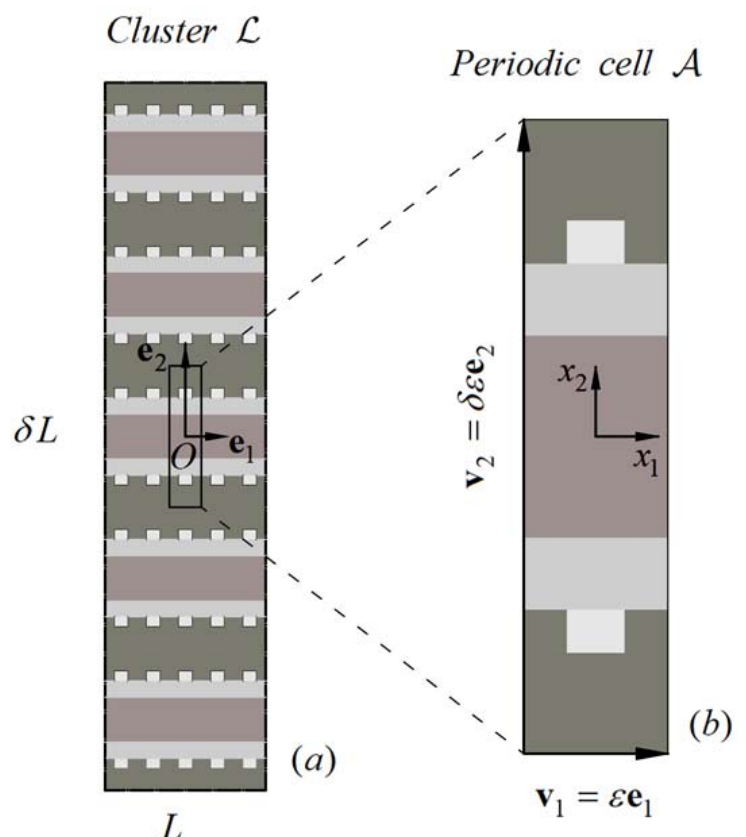

Unit cell $\mathcal{Q}$

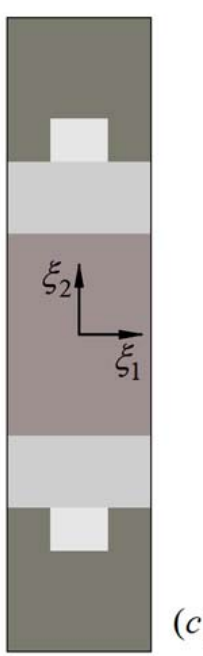

Figure 1: (a) Cluster $\mathcal{L}$ of heterogeneous $S O F C$ having structural characteristic size L; (b) periodic cell $\mathcal{A}$ with microstructural characteristic size $\varepsilon$ and periodicity vectors $\mathbf{v}_{1}$ and $\mathbf{v}_{2} ;$; (c) unit cell $\mathcal{Q}$.

$\boldsymbol{D}^{(m, \varepsilon)}=D_{i j}^{m}\left(\boldsymbol{\xi}=\frac{\mathbf{x}}{\varepsilon}\right) \mathbf{e}_{i} \otimes \mathbf{e}_{j}$ is the symmetric second order micro mass diffusion tensor. Micro constitutive tensors are all $\mathcal{Q}$-periodic and dependent upon the fast variable $\boldsymbol{\xi}$. Local balance equations hold

$$
\begin{aligned}
& \nabla \cdot \boldsymbol{\sigma}(\mathbf{x}, t)+\mathbf{b}(\mathbf{x}, t)=\rho^{m} \ddot{\mathbf{u}}(\mathbf{x}, t), \\
& \nabla \cdot \boldsymbol{q}(\mathbf{x}, t)-r(x)=-\boldsymbol{\alpha}^{m} \dot{\boldsymbol{\varepsilon}}(\mathbf{x}, t)-\psi^{m} \dot{\eta}(\mathbf{x}, t)-p^{m} \dot{\theta}(\mathbf{x}, t), \\
& \nabla \cdot \mathbf{j}(\mathbf{x}, t)-s(\mathbf{x}, t)=-\boldsymbol{\beta}^{m} \dot{\boldsymbol{\varepsilon}}(\mathbf{x}, t)-\psi^{m} \dot{\theta}(\mathbf{x}, t)-q^{m} \dot{\eta}(\mathbf{x}, t),
\end{aligned}
$$

where source terms depend exclusively upon the slow variable and time and are represented by body forces $\mathbf{b}(\mathbf{x}, t)$, heat sources $r(\mathbf{x}, t)$, and mass sources $s(\mathbf{x}, t)$. Source terms are here assumed to be $\mathcal{L}$-periodic and to have vanishing mean values on $\mathcal{L}$, where, indicating with $L$ the structural characteristic size, the portion $\mathcal{L}=[0, L] \times[0, \delta L]$ can be considered as truly representative of the whole medium. In this regard, size $L$ has to be much greater than the microstructural one $(L \gg \varepsilon)$ so that the scales separation condition is met. In equations 2 2a)- $2 \mathrm{c})$ inertial terms are $\mathcal{Q}$-periodic and represented by the mass density $\rho^{(m, \varepsilon)}=$ $\rho^{m}\left(\boldsymbol{\xi}=\frac{\mathbf{x}}{\varepsilon}\right)$, material constant $p^{(m, \varepsilon)}=p^{m}\left(\boldsymbol{\xi}=\frac{\mathbf{x}}{\varepsilon}\right)$ related to the specific heat at constant strain and to thermo-diffusive effects, and material constant $q^{(m, \varepsilon)}=q^{m}\left(\boldsymbol{\xi}=\frac{\mathbf{x}}{\varepsilon}\right)$ related to diffusive effect. Finally, term $\psi^{(m, \varepsilon)}=\psi^{m}\left(\boldsymbol{\xi}=\frac{\mathbf{x}}{\varepsilon}\right)$ is a $\mathcal{Q}$-periodic coupling constant measuring the thermo-diffusive effect. Substitution of constitutive equations (1a)- $1 \mathrm{c}$ ) into local balance relations $(2 \mathrm{a})-(2 \mathrm{c})$ leads to

$$
\begin{aligned}
& \nabla \cdot\left(\mathfrak{C}^{m}\left(\frac{\mathbf{x}}{\varepsilon}\right) \nabla \mathbf{u}(\mathbf{x}, t)\right)-\nabla \cdot\left(\boldsymbol{\alpha}^{m}\left(\frac{\mathbf{x}}{\varepsilon}\right) \theta(\mathbf{x}, t)\right)-\nabla \cdot\left(\boldsymbol{\beta}^{m}\left(\frac{\mathbf{x}}{\varepsilon}\right) \eta(\mathbf{x}, t)\right)+\mathbf{b}(\mathbf{x}, t)=\rho^{m}\left(\frac{\mathbf{x}}{\varepsilon}\right) \ddot{\mathbf{u}}(\mathbf{x}, t), \\
& \nabla \cdot\left(\boldsymbol{K}^{m}\left(\frac{\mathbf{x}}{\varepsilon}\right) \nabla \theta(\mathbf{x}, t)\right)-\boldsymbol{\alpha}^{m}\left(\frac{\mathbf{x}}{\varepsilon}\right) \nabla \dot{\boldsymbol{u}}(\mathbf{x}, t)-\psi^{m}\left(\frac{\mathbf{x}}{\varepsilon}\right) \dot{\eta}(\mathbf{x}, t)+r(\mathbf{x}, t)=p^{m}\left(\frac{\mathbf{x}}{\varepsilon}\right) \dot{\theta}(\mathbf{x}, t), \\
& \nabla \cdot\left(\mathbf{D}^{m}\left(\frac{\mathbf{x}}{\varepsilon}\right) \nabla \eta(\mathbf{x}, t)\right)-\boldsymbol{\beta}^{m}\left(\frac{\mathbf{x}}{\varepsilon}\right) \nabla \dot{\boldsymbol{u}}(\mathbf{x}, t)-\psi^{m}\left(\frac{\mathbf{x}}{\varepsilon}\right) \dot{\theta}(\mathbf{x}, t)+s(\mathbf{x}, t)=q^{m}\left(\frac{\mathbf{x}}{\varepsilon}\right) \dot{\eta}(\mathbf{x}, t) .
\end{aligned}
$$

For an ideally bonded interface $\Sigma$, the following continuity conditions hold

$$
\begin{array}{ll}
{\left.\left[\left[u_{i}\right]\right]\right|_{\mathbf{x} \in \Sigma}=0,} & {\left.\left[\left[\left(C_{i j k l}^{m} \frac{\partial u_{k}}{\partial x_{l}}-\alpha_{i j}^{m} \theta-\beta_{i j}^{m} \eta\right) n_{j}\right]\right]\right|_{\mathbf{x} \in \Sigma}=0,} \\
{\left.[[\theta]]\right|_{\mathbf{x} \in \Sigma}=0,} & {\left.\left[\left[K_{i j}^{m} \frac{\partial \theta}{\partial x_{j}} n_{i}\right]\right]\right|_{\mathbf{x} \in \Sigma}=0}
\end{array}
$$




$$
\left.[[\eta]]\right|_{\mathbf{x} \in \Sigma}=0,\left.\quad\left[\left[D_{i j}^{m} \frac{\partial \eta}{\partial x_{j}} n_{i}\right]\right]\right|_{\mathbf{x} \in \Sigma}=0
$$

where $[[f]]=f^{i}(\Sigma)-f^{j}(\Sigma)$ denotes the discontinuity of the values of a function $f$ at the interface $\Sigma$ between two different phases $i$ and $j$ of periodic cell $\mathcal{A}$ and $\mathbf{n}=n_{j} \mathbf{e}_{j}$ represents the outward normal to the interface $\Sigma$.

Taking into account the $\mathcal{Q}$-periodicity of micro constitutive tensors and inertial terms, interface conditions (4a)-(4c), and the $\mathcal{L}$-periodicity of source terms, it results that the microscopic fields spatially depend on both the slow and the fast variables $\mathbf{x}$ and $\boldsymbol{\xi}$ and are expressed as

$$
\mathbf{u}=\mathbf{u}\left(\mathbf{x}, \boldsymbol{\xi}=\frac{\mathbf{x}}{\varepsilon}, t\right), \quad \theta=\theta\left(\mathbf{x}, \boldsymbol{\xi}=\frac{\mathbf{x}}{\varepsilon}, t\right), \quad \eta=\eta\left(\mathbf{x}, \boldsymbol{\xi}=\frac{\mathbf{x}}{\varepsilon}, t\right) .
$$

Rapidly oscillating $\mathcal{Q}$-periodic coefficients of PDEs (3a)-33c make their analytical and/or numerical resolution particularly labor intensive. In this sense, homogenization techniques can reveal very useful in replacing the microstructured continuum with an equivalent homogeneous one. In what follows, field equations of a first-order thermo-diffusive equivalent continuum will be characterized and the closed form of overall constitutive tensors will be obtained. By means of a dynamic multi-field asymptotic homogenization technique, the global behavior of the composite material will be concisely and accurately described, thus overcoming the computational burden of resolution of equations $(3 \mathrm{a})-(3 \mathrm{c})$ and facilitating their analytical resolution on simple domains. Macroscopic fields of the equivalent homogenized medium, are denoted as $\mathbf{U}(\mathbf{x}, t)=U_{i}(\mathbf{x}, t) \mathbf{e}_{i}$ for the displacement, $\Theta(\mathbf{x}, t)$ for the relative temperature and $\Upsilon(\mathbf{x}, t)$ for chemical potential. They only depend in space upon the macroscopic slow variable $\mathbf{x}$ and they result to be $\mathcal{L}$-periodic if source terms are $\mathcal{L}$-periodic.

\subsection{Asymptotic expansion of field equations at the microscale for the thermo- diffusive medium}

In accordance with the procedure described in (Bensoussan et al., 1978, Bakhvalov and Panasenko, 1984), an asymptotic expansion of the microfields $\mathbf{u}(\mathbf{x}, \mathbf{x} / \varepsilon, t), \theta(\mathbf{x}, \mathbf{x} / \varepsilon, t)$ and $\eta(\mathbf{x}, \mathbf{x} / \varepsilon, t)$ is performed in powers of the micro structural size $\varepsilon$

$$
\begin{aligned}
& u_{h}\left(\mathbf{x}, \frac{\mathbf{x}}{\varepsilon}, t\right)=\sum_{l=0}^{+\infty} \varepsilon^{l} u_{h}^{(l)}\left(\mathbf{x}, \frac{\mathbf{x}}{\varepsilon}, t\right)=u_{h}^{(0)}\left(\mathbf{x}, \frac{\mathbf{x}}{\varepsilon}, t\right)+\varepsilon u_{h}^{(1)}\left(\mathbf{x}, \frac{\mathbf{x}}{\varepsilon}, t\right)+\varepsilon^{2} u_{h}^{(2)}\left(\mathbf{x}, \frac{\mathbf{x}}{\varepsilon}, t\right)+O\left(\varepsilon^{3}\right), \\
& \theta\left(\mathbf{x}, \frac{\mathbf{x}}{\varepsilon}, t\right)=\sum_{l=0}^{+\infty} \varepsilon^{l} \theta^{(l)}\left(\mathbf{x}, \frac{\mathbf{x}}{\varepsilon}, t\right)=\theta^{(0)}\left(\mathbf{x}, \frac{\mathbf{x}}{\varepsilon}, t\right)+\varepsilon \theta^{(1)}\left(\mathbf{x}, \frac{\mathbf{x}}{\varepsilon}, t\right)+\varepsilon^{2} \theta^{(2)}\left(\mathbf{x}, \frac{\mathbf{x}}{\varepsilon}, t\right)+O\left(\varepsilon^{3}\right), \\
& \eta\left(\mathbf{x}, \frac{\mathbf{x}}{\varepsilon}, t\right)=\sum_{l=0}^{+\infty} \varepsilon^{l} \eta^{(l)}\left(\mathbf{x}, \frac{\mathbf{x}}{\varepsilon}, t\right)=\eta^{(0)}\left(\mathbf{x}, \frac{\mathbf{x}}{\varepsilon}, t\right)+\varepsilon \eta^{(1)}\left(\mathbf{x}, \frac{\mathbf{x}}{\varepsilon}, t\right)+\varepsilon^{2} \eta^{(2)}\left(\mathbf{x}, \frac{\mathbf{x}}{\varepsilon}, t\right)+O\left(\varepsilon^{3}\right) .
\end{aligned}
$$

Taking into account the property

$$
\frac{D}{D x_{j}} f\left(\mathbf{x}, \boldsymbol{\xi}=\frac{\mathbf{x}}{\varepsilon}, t\right)=\left.\left(\frac{\partial f}{\partial x_{j}}+\frac{1}{\varepsilon} \frac{\partial f}{\partial \xi_{j}}\right)\right|_{\boldsymbol{\xi}=\frac{\mathbf{x}}{\varepsilon}}=\left.\left(\frac{\partial f}{\partial x_{j}}+\frac{1}{\varepsilon} f_{, j}\right)\right|_{\boldsymbol{\xi}=\frac{\mathbf{x}}{\varepsilon}},
$$

asymptotic expansions (6a)- $6 \mathrm{c}$ ) are substituted into the local field equations (3a)-3c). From equation 3a one has

$$
\begin{aligned}
& \left\{\varepsilon^{-2}\left(C_{i j k l}^{m} u_{k, l}^{(0)}\right)_{, j}+\right. \\
& +\varepsilon^{-1}\left\{\left[C_{i j k l}^{m}\left(\frac{\partial u_{k}^{(0)}}{\partial x_{l}}+u_{k, l}^{(1)}\right)\right]_{, j}+\frac{\partial}{\partial x_{j}}\left(C_{i j k l}^{m} u_{k, l}^{(0)}\right)-\left(\alpha_{i j}^{m} \theta^{(0)}\right)_{, j}-\left(\beta_{i j}^{m} \eta^{(0)}\right)_{, j}\right\} \\
& +\left\{\left[C_{i j k l}^{m}\left(\frac{\partial u_{k}^{(1)}}{\partial x_{l}}+u_{k, l}^{(2)}\right)\right]_{, j}+\frac{\partial}{\partial x_{j}}\left[C_{i j k l}^{m}\left(\frac{\partial u_{k}^{(0)}}{\partial x_{l}}+u_{k, l}^{(1)}\right)\right]-\left(\alpha_{i j}^{m} \theta^{(1)}\right)_{, j}-\frac{\partial}{\partial x_{j}}\left(\alpha_{i j}^{m} \theta^{(0)}\right)+\right.
\end{aligned}
$$




$$
\begin{aligned}
& \left.-\left(\beta_{i j}^{m} \eta^{(1)}\right)_{, j}-\frac{\partial}{\partial x_{j}}\left(\beta_{i j}^{m} \eta^{(0)}\right)\right\}+ \\
& +\varepsilon\left\{\left[C_{i j k l}^{m}\left(\frac{\partial u_{k}^{(2)}}{\partial x_{l}}+u_{k, l}^{(3)}\right)\right]_{, j}+\frac{\partial}{\partial x_{j}}\left[C_{i j k l}^{m}\left(\frac{\partial u_{k}^{(1)}}{\partial x_{l}}+u_{k, l}^{(2)}\right)\right]-\left(\alpha_{i j}^{m} \theta^{(2)}\right)_{, j}-\frac{\partial}{\partial x_{j}}\left(\alpha_{i j}^{m} \theta^{(1)}\right)+\right. \\
& \left.-\left(\beta_{i j}^{m}, \eta^{(2)}\right)_{, j}-\frac{\partial}{\partial x_{j}}\left(\beta_{i j}^{m} \eta^{(1)}\right)-\rho^{m} \frac{\partial^{2} u_{i}^{(0)}}{\partial t^{2}}-\varepsilon \rho^{m} \frac{\partial^{2} u_{i}^{(1)}}{\partial t^{2}}+O\left(\varepsilon^{2}\right)\right\}\left.\right|_{\xi=\frac{\mathbf{x}}{\varepsilon}}+b_{i}(\mathbf{x}, t)=0 .
\end{aligned}
$$

Analogously, field equation (3b) leads to

$$
\begin{aligned}
& \left\{\varepsilon^{-2}\left(K_{i j}^{m} \theta_{, j}^{(0)}\right)_{, i}+\varepsilon^{-1}\left\{\left[K_{i j}^{m}\left(\frac{\partial \theta^{(0)}}{\partial x_{j}}+\theta_{, j}^{(1)}\right)\right]_{, i}+\frac{\partial}{\partial x_{i}}\left(K_{i j}^{m} \theta_{, j}^{(0)}\right)-\alpha_{i j}^{m} \frac{\partial u_{i, j}^{(0)}}{\partial t}\right\}+\right. \\
& +\left[K_{i j}^{m}\left(\frac{\partial \theta^{(1)}}{\partial x_{j}}+\theta_{, j}^{(2)}\right)\right]_{, i}+\frac{\partial}{\partial x_{i}}\left[K_{i j}^{m}\left(\frac{\partial \theta^{(0)}}{\partial x_{j}}+\theta_{, j}^{(1)}\right)\right]-\alpha_{i j}^{m}\left[\frac{\partial^{2} u_{i}^{(0)}}{\partial x_{j} \partial t}+\frac{\partial u_{i, j}^{(1)}}{\partial t}\right]-\psi^{m} \frac{\partial \eta^{(0)}}{\partial t}+ \\
& +\varepsilon\left\{\left[K_{i j}^{m}\left(\frac{\partial \theta^{(2)}}{\partial x_{j}}+\theta_{, j}^{(3)}\right)\right]_{, i}+\frac{\partial}{\partial x_{i}}\left[K_{i j}^{m}\left(\frac{\partial \theta^{(1)}}{\partial x_{j}}+\theta_{, j}^{(2)}\right)\right]-\alpha_{i j}^{m}\left[\frac{\partial^{2} u_{i}^{(1)}}{\partial x_{j} \partial t}+\frac{\partial u_{i, j}^{(2)}}{\partial t}\right]-\psi^{m} \frac{\partial \eta^{(1)}}{\partial t}+\right. \\
& \left.-p^{m} \frac{\partial \theta^{(0)}}{\partial t}-\varepsilon p^{m} \frac{\partial \theta^{(1)}}{\partial t} O\left(\varepsilon^{2}\right)\right\}\left.\right|_{\boldsymbol{\xi}=\frac{\mathbf{x}}{\varepsilon}}+r(\mathbf{x}, t)=0,
\end{aligned}
$$

and equation (3c) results

$$
\begin{aligned}
& \left\{\varepsilon^{-2}\left(D_{i j}^{m} \eta_{, j}^{(0)}\right)_{, i}+\varepsilon^{-1}\left\{\left[D_{i j}^{m}\left(\frac{\partial \eta^{(0)}}{\partial x_{j}}+\eta_{, j}^{(1)}\right)\right]_{, i}+\frac{\partial}{\partial x_{i}}\left(D_{i j}^{m} \eta_{, j}^{(0)}\right)-\beta_{i j}^{m} \frac{\partial u_{i, j}^{(0)}}{\partial t}\right\}+\right. \\
& +\left[D_{i j}^{m}\left(\frac{\partial \eta^{(1)}}{\partial x_{j}}+\eta_{, j}^{(2)}\right)\right]_{, i}+\frac{\partial}{\partial x_{i}}\left[D_{i j}^{m}\left(\frac{\partial \eta^{(0)}}{\partial x_{j}}+\eta_{, j}^{(1)}\right)\right]-\beta_{i j}^{m}\left[\frac{\partial^{2} u_{i}^{(0)}}{\partial x_{j} \partial t}+\frac{\partial u_{i, j}^{(1)}}{\partial t}\right]-\psi^{m} \frac{\partial \theta^{(0)}}{\partial t}+ \\
& +\varepsilon\left\{\left[D_{i j}^{m}\left(\frac{\partial \eta^{(2)}}{\partial x_{j}}+\eta_{, j}^{(3)}\right)\right]_{, i}+\frac{\partial}{\partial x_{i}}\left[D_{i j}^{m}\left(\frac{\partial \eta^{(1)}}{\partial x_{j}}+\eta_{, j}^{(2)}\right)\right]-\beta_{i j}^{m}\left[\frac{\partial^{2} u_{i}^{(1)}}{\partial x_{j} \partial t}+\frac{\partial u_{i, j}^{(2)}}{\partial t}\right]-\psi^{m} \frac{\partial \theta^{(1)}}{\partial t}+\right. \\
& \left.-q^{m} \frac{\partial \eta^{(0)}}{\partial t}-\varepsilon q^{m} \frac{\partial \eta^{(1)}}{\partial t}+O\left(\varepsilon^{2}\right)\right\}\left.\right|_{\boldsymbol{\xi}=\frac{\mathbf{x}}{\varepsilon}}+s(\mathbf{x}, t)=0 .
\end{aligned}
$$

Denoting with $\Sigma_{1}$ the interface between two distinct phases in the unit cell $\mathcal{Q}$, asymptotic expansions (6a)(6c) allow rephrasing interface conditions (4a)- 4 c) over the unit cell $\mathcal{Q}$ in terms of the fast variable $\boldsymbol{\xi}$ (Bakhvalov and Panasenko, 1984). In particular, equations 4a) become

$$
\begin{aligned}
& \left.\left.\left[\left[u_{h}^{(0)}\right]\right]\right|_{\boldsymbol{\xi} \in \Sigma_{1}}+\varepsilon\left[\left[u_{h}^{(1)}\right]\right]\right]\left.\right|_{\boldsymbol{\xi} \in \Sigma_{1}}+O\left(\varepsilon^{2}\right)=0 \\
& \left.\frac{1}{\varepsilon}\left[\left[C_{i j k l}^{m} u_{k, l}^{(0)} n_{j}\right]\right]\right|_{\boldsymbol{\xi} \in \Sigma_{1}}+\left.\left[\left[\left\{C_{i j k l}^{m}\left(\frac{\partial u_{k}^{(0)}}{\partial x_{l}}+u_{k, l}^{(1)}\right)-\alpha_{i j}^{m} \theta^{(0)}-\beta_{i j}^{m} \eta^{(0)}\right\} n_{j}\right]\right]\right|_{\boldsymbol{\xi} \in \Sigma_{1}}+ \\
& +\left.\varepsilon\left[\left[\left\{C_{i j k l}^{m}\left(\frac{\partial u_{k}^{(1)}}{\partial x_{l}}+u_{k, l}^{(2)}\right)-\alpha_{i j}^{m} \theta^{(1)}-\beta_{i j}^{m} \eta^{(1)}\right\} n_{j}\right]\right]\right|_{\boldsymbol{\xi} \in \Sigma_{1}}+O\left(\varepsilon^{2}\right)=0
\end{aligned}
$$

equations $4 \mathrm{~b}$ are written as

$$
\begin{aligned}
& {\left.\left[\left[\theta^{(0)}\right]\right]\right|_{\boldsymbol{\xi} \in \Sigma_{1}}+\left.\varepsilon\left[\left[\theta^{(1)}\right]\right]\right|_{\boldsymbol{\xi} \in \Sigma_{1}}+O\left(\varepsilon^{2}\right)=0,} \\
& \left.\frac{1}{\varepsilon}\left[\left[K_{i j}^{m} \theta_{, j}^{(0)} n_{i}\right]\right]\right|_{\boldsymbol{\xi} \in \Sigma_{1}}+\left.\left[\left[K_{i j}^{m}\left(\frac{\partial \theta^{(0)}}{\partial x_{j}}+\theta_{, j}^{(1)}\right) n_{i}\right]\right]\right|_{\boldsymbol{\xi} \in \Sigma_{1}}+
\end{aligned}
$$




$$
+\left.\varepsilon\left[\left[K_{i j}^{m}\left(\frac{\partial \theta^{(1)}}{\partial x_{j}}+\theta_{, j}^{(2)}\right) n_{i}\right]\right]\right|_{\boldsymbol{\xi} \in \Sigma_{1}}+O\left(\varepsilon^{2}\right)=0,
$$

and interface conditions $4 \mathrm{c}$ involving chemical potential turn into

$$
\begin{aligned}
& {\left.\left[\left[\eta^{(0)}\right]\right]\right|_{\boldsymbol{\xi} \in \Sigma_{1}}+\left.\varepsilon\left[\left[\eta^{(1)}\right]\right]\right|_{\boldsymbol{\xi} \in \Sigma_{1}}+O\left(\varepsilon^{2}\right)=0} \\
& \left.\frac{1}{\varepsilon}\left[\left[D_{i j}^{m} \eta_{, j}^{(0)} n_{i}\right]\right]\right|_{\boldsymbol{\xi} \in \Sigma_{1}}+\left.\left[\left[D_{i j}^{m}\left(\frac{\partial \eta^{(0)}}{\partial x_{j}}+\eta_{, j}^{(1)}\right) n_{i}\right]\right]\right|_{\boldsymbol{\xi} \in \Sigma_{1}}+ \\
& +\left.\varepsilon\left[\left[D_{i j}^{m}\left(\frac{\partial \eta^{(1)}}{\partial x_{j}}+\eta_{, j}^{(2)}\right) n_{i}\right]\right]\right|_{\boldsymbol{\xi} \in \Sigma_{1}}+O\left(\varepsilon^{2}\right)=0 .
\end{aligned}
$$

In the followings, recursive differential problems originating from equations (7)-(9) are written explicitly at the different orders of length $\varepsilon$ till the order $\varepsilon^{0}$, leading to the definition of cell problems in Section 3 .

Recursive differential problems at the order $\varepsilon^{-2}$

From equation (7), at the order $\varepsilon^{-2}$ one has the following differential problem

$$
\left(C_{i j k l}^{m} u_{k, l}^{(0)}\right)_{, j}=f_{i}^{(0)}(\mathbf{x}, t)
$$

with interface conditions

$$
\left.\left[\left[u_{k}^{(0)}\right]\right]\right|_{\boldsymbol{\xi} \in \Sigma_{1}}=0,\left.\quad\left[\left[C_{i j k l}^{m} u_{k, l}^{(0)} n_{j}\right]\right]\right|_{\boldsymbol{\xi} \in \Sigma_{1}}=0
$$

It results that $f_{i}^{(0)}=0$ in equation 13 because of solvability condition of problem 13 in the class of $\mathcal{Q}$-periodic functions and interface conditions 14, (Bakhvalov and Panasenko, 1984), and the solution $u_{k}^{(0)}$ spatially depends only upon the slow variable $\mathbf{x}$, being equal to the macroscopic field

$$
u_{k}^{(0)}(\mathbf{x}, \boldsymbol{\xi}, t)=U_{k}(\mathbf{x}, t)
$$

At the order $\varepsilon^{-2}$, from equation (8) one has

$$
\left(K_{i j}^{m} \theta_{, j}^{(0)}\right)_{, i}=g^{(0)}(\mathbf{x}, t)
$$

with relative interface conditions from (11) that hold

$$
\left.\left[\left[\theta^{(0)}\right]\right]\right|_{\boldsymbol{\xi} \in \Sigma_{1}}=0,\left.\quad\left[\left[K_{i j}^{m} \theta_{, j}^{(0)} n_{i}\right]\right]\right|_{\boldsymbol{\xi} \in \Sigma_{1}}=0
$$

For the same reasons explicited above, the solution $\theta^{(0)}$ is equal to the macroscopic temperature field, namely

$$
\theta^{(0)}(\mathbf{x}, \boldsymbol{\xi}, t)=\Theta(\mathbf{x}, t)
$$

Analogously, from equation 9 differential problem obtained at the order $\varepsilon^{-2}$ has the form

$$
\left(D_{i j}^{m} \eta_{, j}^{(0)}\right)_{, i}=h^{(0)}(\mathbf{x})
$$

with relative interface conditions from equation 12 that read

$$
\left.\left[\left[\eta^{(0)}\right]\right]\right|_{\boldsymbol{\xi} \in \Sigma_{1}}=0,\left.\quad\left[\left[D_{i j}^{m} \eta_{, j}^{(0)} n_{i}\right]\right]\right|_{\boldsymbol{\xi} \in \Sigma_{1}}=0
$$

Once again, solution of 19 corresponds to the macroscopic chemical potential and it is expressed as

$$
\eta^{(0)}(\mathbf{x}, \boldsymbol{\xi}, t)=\Upsilon(\mathbf{x}, t)
$$


Recursive differential problems at the order $\varepsilon^{-1}$

Taking into account solutions (15), 18), and 21) of problems at the order $\varepsilon^{-2}$, at the order $\varepsilon^{-1}$ from equation (7) one has the following differential problem

$$
\left(C_{i j k l}^{m} u_{k, l}^{(1)}\right)_{, j}+\left(C_{i j k l}^{m} \frac{\partial U_{k}}{\partial x_{l}}\right)_{, j}-\alpha_{i j, j}^{m} \Theta-\beta_{i j, j}^{m} \Upsilon=f_{i}^{(1)}(\mathbf{x}, t),
$$

with interface conditions expressed as

$$
\left.\left[\left[u_{h}^{(1)}\right]\right]\right|_{\boldsymbol{\xi} \in \Sigma_{1}}=0,\left.\quad\left[\left[\left\{C_{i j k l}^{m}\left(\frac{\partial U_{k}}{\partial x_{l}}+u_{k, l}^{(1)}\right)-\alpha_{i j}^{m} \Theta-\beta_{i j}^{m} \Upsilon\right\} n_{j}\right]\right]\right|_{\boldsymbol{\xi} \in \Sigma_{1}}=0 .
$$

Given the $Q$-periodicity of components $C_{i j k l}^{m}, \alpha_{i j}^{m}$, and $\beta_{i j}^{m}$, solvability condition of problem 22 imposes that

$$
f_{i}^{(1)}(\mathbf{x}, t)=\left\langle C_{i j k l, j}^{m}\right\rangle \frac{\partial U_{k}}{\partial x_{l}}-\left\langle\alpha_{i j, j}^{m}\right\rangle \Theta-\left\langle\beta_{i j, j}^{m}\right\rangle \Upsilon
$$

where $\langle(\cdot)\rangle=\frac{1}{\mid \mathcal{Q}} \mid \int_{\mathcal{Q}}(\cdot) d \boldsymbol{\xi}$ and $|\mathcal{Q}|=\delta$ denotes the area of the unit cell. Solutions $[15,,[18$, , and $[21)$, make the micro displacement solution at the order $\varepsilon^{-1}$ of the form

$$
u_{k}^{(1)}(\mathbf{x}, \boldsymbol{\xi}, t)=N_{k p q_{1}}^{(1)}(\boldsymbol{\xi}) \frac{\partial U_{p}(\mathbf{x}, t)}{\partial x_{q_{1}}}+\tilde{N}_{k}^{(1)}(\boldsymbol{\xi}) \Theta(\mathbf{x}, t)+\hat{N}_{k}^{(1)}(\boldsymbol{\xi}) \Upsilon(\mathbf{x}, t),
$$

where $N_{k p q_{1}}^{(1)}, \tilde{N}_{k}^{(1)}$, and $\hat{N}_{k}^{(1)}$ are the first-order perturbation functions for the mechanical problem. These are $\mathcal{Q}$-periodic functions and reflect the effects of the underlying microstructure being spatially dependent only upon $\boldsymbol{\xi}$. At the order $\varepsilon^{-1}$, from equation (8) one obtains

$$
\left(K_{i j}^{m} \theta_{, j}^{(1)}\right)_{, i}+\left(K_{i j}^{m} \frac{\partial \Theta}{\partial x_{j}}\right)_{, i}=g^{(1)}(\mathbf{x}, t)
$$

and relative interface conditions from (11) read

$$
\left.\left[\left[\theta^{(1)}\right]\right]\right|_{\boldsymbol{\xi} \in \Sigma_{1}}=0,\left.\quad\left[\left[K_{i j}^{m}\left(\frac{\partial \Theta}{\partial x_{j}}+\theta_{, j}^{(1)}\right) n_{i}\right]\right]\right|_{\boldsymbol{\xi} \in \Sigma_{1}}=0
$$

Solvability of differential problem $\left[26\right.$, taking into account the $\mathcal{Q}$-periodicity of components $K_{i j}^{m}$ leads to

$$
g^{(1)}(\mathbf{x}, t)=\left\langle K_{i j, j}^{m}\right\rangle=0 .
$$

Therefore, solution of 26 has the form

$$
\theta^{(1)}(\mathbf{x}, \boldsymbol{\xi}, t)=M_{q_{1}}^{(1)}(\boldsymbol{\xi}) \frac{\partial \Theta}{\partial x_{q_{1}}},
$$

with perturbation function $M_{q_{1}}^{(1)}$. Analogously to what done for thermal problem, from equation (9) diffusion problem at the order $\varepsilon^{-1}$ has the form

$$
\left(D_{i j}^{m} \eta_{, j}^{(1)}\right)_{, i}+\left(D_{i j}^{m} \frac{\partial \Upsilon}{\partial x_{j}}\right)_{, i}=h^{(1)}(\mathbf{x}, t)
$$

and its interface conditions read

$$
\left.\left[\left[\eta^{(1)}\right]\right]\right|_{\boldsymbol{\xi} \in \Sigma_{1}}=0,\left.\quad\left[\left[D_{i j}^{m}\left(\frac{\partial \Upsilon}{\partial x_{j}}+\eta_{, j}^{(1)}\right) n_{i}\right]\right]\right|_{\boldsymbol{\xi} \in \Sigma_{1}}=0 .
$$

Solvability condition for problem (30) imposes that

$$
h^{(1)}(\mathbf{x}, t)=\left\langle D_{i j, i}^{m}\right\rangle=0
$$


and the solution $h^{(1)}(\mathbf{x}, t)$ has the form

$$
\eta^{(1)}(\mathbf{x}, \boldsymbol{\xi}, t)=W_{q_{1}}^{(1)}(\boldsymbol{\xi}) \frac{\partial \Upsilon(\mathbf{x}, t)}{\partial x_{q_{1}}},
$$

with first-order perturbation function $W_{q_{1}}^{(1)}$.

Recursive differential problems at the order $\varepsilon^{0}$

Bearing in mind the two sets of solutions (15), (18), 21) and (25), 29, , (33) of differential problems at the order $\varepsilon^{-2}$ and $\varepsilon^{-1}$, respectively, equation (7) at the order $\varepsilon^{0}$ yields

$$
\begin{aligned}
& \left(C_{i j k l}^{m}+u_{k, l}^{(2)}\right)_{, j}+\left[\left(C_{i j k l}^{m} N_{k p q_{1}}^{(1)}\right)_{, j}+C_{i q_{1} p l}^{m}+C_{i l k j}^{m} N_{k p q_{1}, j}^{(1)}\right] \frac{\partial^{2} U_{p}}{\partial x_{q_{1}} \partial x_{l}}+ \\
& +\left[\left(C_{i j k l}^{m} \tilde{N}_{k}^{(1)}\right)_{, j}+C_{i l k j}^{m} \tilde{N}_{k, j}^{(1)}-\left(\alpha_{i j}^{m} M_{l}^{(1)}\right)_{, j}-\alpha_{i l}^{m}\right] \frac{\partial \Theta}{\partial x_{l}}+ \\
& +\left[\left(C_{i j k l}^{m} \hat{N}_{k}^{(1)}\right)_{, j}+C_{i l k j}^{m} \hat{N}_{k, j}^{(1)}-\left(\beta_{i j}^{m} W_{l}^{(1)}\right)_{, j}-\beta_{i l}^{m}\right] \frac{\partial \Upsilon}{\partial x_{l}}-\rho^{m} \frac{\partial^{2} U_{i}}{\partial t^{2}}=f_{i}^{(2)}(\mathbf{x}, t),
\end{aligned}
$$

with interface conditions

$$
\begin{aligned}
& {\left.\left[\left[u_{h}^{(2)}\right]\right]\right|_{\boldsymbol{\xi} \in \Sigma_{1}}=0,} \\
& {\left[\left[\left\{C_{i j k l}^{m}\left(u_{k, l}^{(2)}+N_{k p q_{1}}^{(1)} \frac{\partial^{2} U_{p}}{\partial x_{q_{1}} \partial x_{l}}+\tilde{N}_{k}^{(1)} \frac{\partial \Theta}{\partial x_{l}}+\hat{N}_{k}^{(1)} \frac{\partial \Upsilon}{\partial x_{l}}\right)+\right.\right.\right.} \\
& \left.\left.\left.-\alpha_{i j}^{m} \delta_{q_{1} l} M_{q_{1}}^{(1)} \frac{\partial \Theta}{\partial x_{l}}-\beta_{i j}^{m} \delta_{q_{1} l} W_{q_{1}}^{(1)} \frac{\partial \Upsilon}{\partial x_{l}}\right\} n_{j}\right]\right]\left.\right|_{\boldsymbol{\xi} \in \Sigma_{1}}=0 .
\end{aligned}
$$

Solvability condition for problem 34 leads to the following condition for $f_{i}^{(2)}$

$$
f_{i}^{(2)}(\mathbf{x}, t)=\left\langle C_{i q_{1} p l}^{m}+C_{i l k j}^{m} N_{k p q_{1}, j}^{(1)}\right\rangle \frac{\partial^{2} U_{p}}{\partial x_{q_{1}} \partial x_{l}}+\left\langle C_{i l k j}^{m} \tilde{N}_{k, j}^{(1)}-\alpha_{i l}^{m}\right\rangle \frac{\partial \Theta}{\partial x_{l}}+\left\langle C_{i l k j}^{m} \hat{N}_{k, j}^{(1)}-\beta_{i l}^{m}\right\rangle \frac{\partial \Upsilon}{\partial x_{l}}-\left\langle\rho^{m}\right\rangle \frac{\partial^{2} U_{i}}{\partial t^{2}},
$$

and the solution has the form

$$
u_{k}^{(2)}(\mathbf{x}, \boldsymbol{\xi}, t)=N_{k p q_{1} q_{2}}^{(2)}(\boldsymbol{\xi}) \frac{\partial^{2} U_{p}(\mathbf{x}, t)}{\partial x_{q_{1}} \partial x_{q_{2}}}+\tilde{N}_{k q_{1}}^{(2)}(\boldsymbol{\xi}) \frac{\partial \Theta(\mathbf{x}, t)}{\partial x_{q_{1}}}+\hat{N}_{k q_{1}}^{(2)}(\boldsymbol{\xi}) \frac{\partial \Upsilon(\mathbf{x}, t)}{\partial x_{q_{1}}}+N_{k p}^{(2,2)}(\boldsymbol{\xi}) \frac{\partial^{2} U_{p}(\mathbf{x}, t)}{\partial t^{2}},
$$

where $N_{k p q_{1} q_{2}}^{(2)}, \tilde{N}_{k q_{1}}^{(2)}, \hat{N}_{k q_{1}}^{(2)}$, and $N_{k p}^{(2,2)}$ are the second order perturbation functions relative to the mechanical problem. From equation (8), thermal problem at the order $\varepsilon^{0}$ reads

$$
\begin{aligned}
& \left(K_{i j}^{m} \theta_{, j}^{(2)}\right)_{, i}+\left[\left(K_{i j}^{m} M_{q_{1}}^{(1)}\right)_{, i}+K_{q_{1} j}^{m}+K_{j i}^{m} M_{q_{1}, i}^{(1)}\right] \frac{\partial^{2} \Theta}{\partial x_{q_{1}} \partial x_{j}}-\left(\alpha_{i j}^{m} N_{i p q_{1}, j}^{(1)}+\alpha_{p q_{1}}^{m}\right) \frac{\partial^{2} U_{p}}{\partial x_{q_{1}} \partial t}+ \\
& -\left(\alpha_{i j} \tilde{N}_{i, j}^{(1)}+p^{m}\right) \frac{\partial \Theta}{\partial t}-\left(\alpha_{i j}^{m} \hat{N}_{i, j}^{(1)}+\psi^{m}\right) \frac{\partial \Upsilon}{\partial t}=g^{(2)}(\mathbf{x}, t),
\end{aligned}
$$

and relative interface conditions have the following form

$$
\left.\left[\left[\theta^{(2)}\right]\right]\right|_{\xi \in \Sigma_{1}}=0,\left.\quad\left[\left[K_{i j}^{m}\left(\theta_{, j}^{(2)}+M_{q_{1}}^{(1)} \frac{\partial^{2} \Theta}{\partial x_{q_{1}} \partial x_{j}}\right) n_{i}\right]\right]\right|_{\xi \in \Sigma_{1}}=0
$$

Solvability condition for $(38)$ entails that

$$
\begin{aligned}
& g^{(2)}(\mathbf{x}, t)=\left\langle\left(K_{i j}^{m} M_{q_{1}}^{(1)}\right)_{, i}+K_{q_{1} j}^{m}+K_{j i}^{m} M_{q_{1}, i}^{(1)}\right\rangle \frac{\partial^{2} \Theta}{\partial x_{q_{1}} \partial x_{j}}-\left\langle\alpha_{i j}^{m} N_{i p q_{1}, j}^{(1)}+\alpha_{p q_{1}}^{m}\right\rangle \frac{\partial^{2} U_{p}}{\partial x_{q_{1}} \partial t}+ \\
& -\left\langle\alpha_{i j} \tilde{N}_{i, j}^{(1)}+p^{m}\right\rangle \frac{\partial \Theta}{\partial t}-\left\langle\alpha_{i j}^{m} \hat{N}_{i, j}^{(1)}+\psi^{m}\right\rangle \frac{\partial \Upsilon}{\partial t}
\end{aligned}
$$


and solution reads

$$
\theta^{(2)}(\mathbf{x}, \boldsymbol{\xi}, t)=M_{q_{1} q_{2}}^{(2)}(\boldsymbol{\xi}) \frac{\partial^{2} \Theta(\mathbf{x}, t)}{\partial x_{q_{1}} \partial x_{q_{2}}}+\tilde{M}_{p q_{1}}^{(2,1)}(\boldsymbol{\xi}) \frac{\partial^{2} U_{p}(\mathbf{x}, t)}{\partial x_{q_{1}} \partial t}+M^{(2,1)}(\boldsymbol{\xi}) \frac{\partial \Theta(\mathbf{x}, t)}{\partial t}+\hat{M}^{(2,1)}(\boldsymbol{\xi}) \frac{\partial \Upsilon(\mathbf{x}, t)}{\partial t},
$$

with second order perturbation functions $M_{q_{1} q_{2}}^{(2)}, \tilde{M}_{p q_{1}}^{(2,1)}, M^{(2,1)}$ and $\hat{M}^{(2,1)}$. Diffusion problem at the order $\varepsilon^{0}$ results from equation $(9)$ and reads

$$
\begin{aligned}
& \left(D_{i j}^{m} \eta_{, j}^{(2)}\right)_{, i}+\left[\left(D_{i j}^{m} W_{q_{1}}^{(1)}\right)_{, i}+D_{q_{1} j}^{m}+D_{j i}^{m} W_{q_{1}, i}^{(1)}\right] \frac{\partial^{2} \Upsilon}{\partial x_{q_{1}} \partial x_{j}}-\left(\beta_{i j}^{m} N_{i p q_{1}, j}^{(1)}+\beta_{p q_{1}}^{m}\right) \frac{\partial^{2} U_{p}}{\partial x_{q_{1}} \partial t}+ \\
& -\left(\beta_{i j} \hat{N}_{i, j}^{(1)}+q^{m}\right) \frac{\partial \Upsilon}{\partial t}-\left(\beta_{i j}^{m} \tilde{N}_{i, j}^{(1)}+\psi^{m}\right) \frac{\partial \Theta}{\partial t}=h^{(2)}(\mathbf{x}, t),
\end{aligned}
$$

with relative interface conditions from 12 in the form

$$
\left.\left[\left[\eta^{(2)}\right]\right]\right|_{\boldsymbol{\xi} \in \Sigma_{1}}=0,\left.\quad\left[\left[D_{i j}^{m}\left(\eta_{, j}^{(2)}+W_{q_{1}}^{(1)} \frac{\partial^{2} \Upsilon}{\partial x_{q_{1}} \partial x_{j}}\right) n_{i}\right]\right]\right|_{\boldsymbol{\xi} \in \Sigma_{1}}=0 .
$$

Solvability condition for differential problem 42 imposes

$$
\begin{aligned}
& h^{(2)}(\mathbf{x}, t)=\left\langle\left(D_{i j}^{m} W_{q_{1}}^{(1)}\right)_{, i}+D_{q_{1} j}^{m}+D_{j i}^{m} W_{q_{1}, i}^{(1)}\right\rangle \frac{\partial^{2} \Upsilon}{\partial x_{q_{1}} \partial x_{j}}-\left\langle\beta_{i j}^{m} N_{i p q_{1}, j}^{(1)}+\beta_{p q_{1}}^{m}\right\rangle \frac{\partial^{2} U_{p}}{\partial x_{q_{1}} \partial t}+ \\
& -\left\langle\beta_{i j} \hat{N}_{i, j}^{(1)}+q^{m}\right\rangle \frac{\partial \Upsilon}{\partial t}-\left\langle\beta_{i j}^{m} \tilde{N}_{i, j}^{(1)}+\psi^{m}\right\rangle \frac{\partial \Theta}{\partial t},
\end{aligned}
$$

with a solution of the form

$$
\eta^{(2)}(\mathbf{x}, \boldsymbol{\xi}, t)=W_{q_{1} q_{2}}^{(2)}(\boldsymbol{\xi}) \frac{\partial^{2} \Upsilon(\mathbf{x}, t)}{\partial x_{q_{1}} \partial x_{q_{2}}}+\tilde{W}_{p q_{1}}^{(2,1)}(\boldsymbol{\xi}) \frac{\partial^{2} U_{p}(\mathbf{x}, t)}{\partial x_{q_{1}} \partial t}+W^{(2,1)}(\boldsymbol{\xi}) \frac{\partial \Upsilon(\mathbf{x}, t)}{\partial t}+\hat{W}^{(2,1)}(\boldsymbol{\xi}) \frac{\partial \Theta(\mathbf{x}, t)}{\partial t},
$$

where $W_{q_{1} q_{2}}^{(2)}, \tilde{W}_{p q_{1}}^{(2,1)}, W^{(2,1)}$ and $\hat{W}^{(2,1)}$ are the relative second order perturbation functions.

\section{Cell problems and perturbation functions}

Cell problems are non homogeneous recursive differential problems obtained inserting into differential problems (7)-(9) the solutions obtained at the different orders of $\varepsilon$. Cell problems are therefore expressed in terms of perturbation functions which depend on geometrical and physico-mechanical features of the microstructure and reflect the effects of material dishomogeneities on microfields. Solutions of cell problems result to be regular, $\mathcal{Q}$-periodic functions because cell problems are elliptic differential problems in divergence form whose terms have vanishing mean values over $\mathcal{Q}$ (Bakhvalov and Panasenko, 1984). In order to guarantee the uniqueness of cell problems solution, the following normalization condition

$$
\langle(\cdot)\rangle=\frac{1}{|\mathcal{Q}|} \int_{\mathcal{Q}}(\cdot) d \boldsymbol{\xi}=0
$$

is required to be fulfilled by all perturbation functions. In what follows cell problems are described in detail for the mechanical, thermal and mass diffusion problems up to order $\varepsilon^{0}$. Higher order cell problems are obtained following the procedure described below, but their expression is not reported in the present note for brevity.

\section{Mechanical cell problems}

From equation (22), in view of the form of solution 25) one obtains the following three cell problems at the order $\varepsilon^{-1}$. The first one and its relative interface conditions are expressed in terms of perturbation function $N_{k p q_{1}}^{(1)}$ and read

$$
\left(C_{i j k l}^{m} N_{k p q_{1}, l}^{(1)}\right)_{, j}+C_{i j p q_{1}, j}^{m}=0,
$$




$$
\begin{aligned}
& {\left.\left[\left[N_{k p q_{1}}^{(1)}\right]\right]\right|_{\boldsymbol{\xi} \in \Sigma_{1}}=0} \\
& {\left.\left[\left[C_{i j k l}^{m}\left(N_{k p q_{1}, l}^{(1)}+\delta_{l q_{1}} \delta_{k p}\right) n_{j}\right]\right]\right|_{\boldsymbol{\xi} \in \Sigma_{1}},=0}
\end{aligned}
$$

where symbol $\delta_{l q_{1}}$ denotes the Kronecker delta function. The second cell problem and its interface conditions are expressed in terms of $\tilde{N}_{k}^{(1)}$ and have the form

$$
\begin{aligned}
& \left(C_{i j k l}^{m} \tilde{N}_{k, l}^{(1)}\right)_{, j}-\alpha_{i j, j}^{m}=0, \\
& {\left.\left[\left[\tilde{N}_{k}^{(1)}\right]\right]\right|_{\boldsymbol{\xi} \in \Sigma_{1}}=0,} \\
& {\left.\left[\left[\left(C_{i j k l}^{m} \tilde{N}_{k, l}^{(1)}-\alpha_{i j}^{m}\right) n_{j}\right]\right]\right|_{\boldsymbol{\xi} \in \Sigma_{1}}=0 .}
\end{aligned}
$$

Finally, the third cell problem is in terms of $\hat{N}_{k}^{(1)}$ and it is expressed in the following way, together with relative interface conditions

$$
\begin{aligned}
& \left(C_{i j k l}^{m} \hat{N}_{k, l}^{(1)}\right)_{, j}-\beta_{i j, j}^{m}=0, \\
& {\left.\left[\left[\hat{N}_{k}^{(1)}\right]\right]\right|_{\boldsymbol{\xi} \in \Sigma_{1}}=0,} \\
& {\left.\left[\left[\left(C_{i j k l}^{m} \hat{N}_{k, l}^{(1)}-\beta_{i j}^{m}\right) n_{j}\right]\right]\right|_{\boldsymbol{\xi} \in \Sigma_{1}}=0 .}
\end{aligned}
$$

When perturbation functions $N_{k p q_{1}}^{(1)}, \tilde{N}_{k}^{(1)}$, and $\hat{N}_{k}^{(1)}$ are determined as solutions of relative cell problems at the order $\varepsilon^{-1}$, from equation (34) and in consideration of the form of the solution (37) one obtains the following four cell problems at the order $\varepsilon^{0}$. The first one is written in a symmetrized from with respect to indices $q_{1}$ and $q_{2}$ and, together with its interface conditions, is here formulated in terms of second order perturbation function $N_{k p q_{1} q_{2}}^{(2)}$ and reads

$$
\begin{aligned}
& \left(C_{i j k l}^{m} N_{k p q_{1} q_{2}, l}^{(2)}\right)_{, j}+\frac{1}{2}\left[\left(C_{i j k q_{2}}^{m} N_{k p q_{1}}^{(1)}+C_{i j k q_{1}}^{m} N_{k p q_{2}}^{(1)}\right)_{, j}+C_{i q_{1} p q_{2}}^{m}+C_{i q_{2} p q_{1}}^{m}+\right. \\
& \left.+C_{i q_{2} k j}^{m} N_{k p q_{1}, j}^{(1)}+C_{i q_{1} k j}^{m} N_{k p q_{2}, j}^{(1)}\right]=\frac{1}{2}\left\langle C_{i q_{1} p q_{2}}^{m}+C_{i q_{2} k j}^{m} N_{k p q_{1}, j}^{(1)}+C_{i q_{2} p q_{1}}^{m}+C_{i q_{1} k j}^{m} N_{k p q_{2}, j}^{(1)}\right\rangle, \\
& {\left.\left[\left[N_{k p q_{1} q_{2}}^{(2)}\right]\right]\right|_{\boldsymbol{\xi} \in \Sigma_{1}}=0} \\
& {\left.\left[\left[C_{i j k l}^{m}\left\{N_{k p q_{1} q_{2}, l}^{(2)}+\frac{1}{2}\left(\delta_{q_{2} l} N_{k p q_{1}}^{(1)}+\delta_{q_{1} l} N_{k p q_{2}}^{(1)}\right)\right\} n_{j}\right]\right]\right|_{\boldsymbol{\xi} \in \Sigma_{1}}=0 .}
\end{aligned}
$$

The second cell problem deriving from 34 and its interface condition involve perturbation function $\tilde{N}_{k q_{1}}^{(2)}$ and are expressed as

$$
\begin{aligned}
& \left(C_{i j k l}^{m} \tilde{N}_{k q_{1}, l}^{(2)}\right)_{, j}+\left[\left(C_{i j k q_{1}}^{m} \tilde{N}_{k}^{(1)}\right)_{, j}+C_{i q_{1} k j}^{m} \tilde{N}_{k, j}^{(1)}-\left(\alpha_{i j}^{m} M_{q_{1}}^{(1)}\right)_{, j}-\alpha_{i q_{1}}^{m}\right]=\left\langle C_{i q_{1} k j}^{m} \tilde{N}_{k, j}^{(1)}-\alpha_{i q_{1}}^{m}\right\rangle, \\
& {\left.\left[\left[\tilde{N}_{k q_{1}}^{(2)}\right]\right]\right|_{\xi \in \Sigma_{1}}=0} \\
& {\left.\left[\left[\left\{C_{i j k l}^{m}\left(\tilde{N}_{k q_{1}}^{(2)}+\delta_{q_{1} l} \tilde{N}_{k}^{(1)}\right)-\alpha_{i j}^{m} M_{q_{1}}^{(1)}\right\} n_{j}\right]\right]\right|_{\boldsymbol{\xi} \in \Sigma_{1}}=0 .}
\end{aligned}
$$

The form of the third cell problem from 34 and its interface conditions in terms of $\hat{N}_{k q_{1}}^{(2)}$ is

$$
\begin{aligned}
& \left(C_{i j k l}^{m} \hat{N}_{k q_{1}, l}^{(2)}\right)_{, j}+\left[\left(C_{i j k q_{1}}^{m} \hat{N}_{k}^{(1)}\right)_{, j}+C_{i q_{1} k j}^{m} \hat{N}_{k, j}^{(1)}-\left(\beta_{i j}^{m} W_{q_{1}}^{(1)}\right)_{, j}-\beta_{i q_{1}}^{m}\right]=\left\langle C_{i q_{1} k j}^{m} \hat{N}_{k, j}^{(1)}-\beta_{i q_{1}}^{m}\right\rangle, \\
& {\left.\left[\left[\hat{N}_{k q_{1}}^{(2)}\right]\right]\right|_{\xi \in \Sigma_{1}}=0,} \\
& {\left.\left[\left[\left\{C_{i j k l}^{m}\left(\hat{N}_{k q_{1}}^{(2)}+\delta_{q_{1} l} \hat{N}_{k}^{(1)}\right)-\beta_{i j}^{m} W_{q_{1}}^{(1)}\right\} n_{j}\right]\right]\right|_{\xi \in \Sigma_{1}}=0 .}
\end{aligned}
$$


The last mechanical cell problem at the order $\varepsilon^{0}$ and its interface conditions have the following expression in terms of perturbation function $N_{k p}^{(2,2)}$

$$
\begin{aligned}
& \left(C_{i j k l}^{m} N_{k p, l}^{(2,2)}\right)_{, j}-\rho^{m} \delta_{i p}=-\left\langle\rho^{m}\right\rangle \delta_{i p}, \\
& {\left.\left[\left[N_{k p}^{(2,2)}\right]\right]\right|_{\boldsymbol{\xi} \in \Sigma_{1}}=0} \\
& {\left.\left[\left[C_{i j k l}^{m} \hat{N}_{k p}^{(2,2)} n_{j}\right]\right]\right|_{\boldsymbol{\xi} \in \Sigma_{1}}=0 .}
\end{aligned}
$$

\section{Thermal cell problems}

From equation (26) and taking into account solution (29), one derives the following cell problem at the order $\varepsilon^{-1}$ which, together with relative interface conditions, provides perturbation function $M_{q_{1}}^{(1)}$

$$
\begin{aligned}
& \left(K_{i j}^{m} M_{q_{1}, j}^{(1)}\right)_{, i}+K_{i q_{1}, i}^{m}=0, \\
& {\left.\left[\left[M_{q_{1}}^{(1)}\right]\right]\right|_{\boldsymbol{\xi} \in \Sigma_{1}}=0,} \\
& {\left.\left[\left[K_{i j}^{m}\left(M_{q_{1}, j}^{(1)}+\delta_{q_{1} j}\right) n_{i}\right]\right]\right|_{\boldsymbol{\xi} \in \Sigma_{1}}=0 .}
\end{aligned}
$$

Once first-order perturbation function $M_{q_{1}}^{(1)}$ is known, four cell problems are derived at the order $\varepsilon^{0}$ from equation (38), bearing in mind solution (41). The first one provides second order perturbation function $M_{q_{1} q_{2}}^{(2)}$ and it is here written in a symmetrized form with respect to indices $q_{1}$ and $q_{2}$, together with relative interface conditions

$$
\begin{aligned}
& \left(K_{i j}^{m} M_{q_{1} q_{2}, j}^{(2)}\right)_{, i}+\frac{1}{2}\left[\left(K_{i q_{2}}^{m} M_{q_{1}}^{(1)}\right)_{, i}+K_{q_{1} q_{2}}^{m}+K_{q_{2} i}^{m} M_{q_{1}, i}^{(1)}+\right. \\
& \left.\left(K_{i q_{1}}^{m} M_{q_{2}}^{(1)}\right)_{, i}+K_{q_{2} q_{1}}^{m}+K_{q_{1} i}^{m} M_{q_{2}, i}^{(1)}\right]=\frac{1}{2}\left\langle K_{q_{1} q_{2}}^{m}+K_{q_{2} i}^{m} M_{q_{1}, i}^{(1)}+K_{q_{2} q_{1}}^{m}+K_{q_{1} i}^{m} M_{q_{2}, i}^{(1)}\right\rangle \\
& {\left.\left[\left[M_{q_{1} q_{2}}^{(2)}\right]\right]\right|_{\xi \in \Sigma_{1}}=0} \\
& {\left.\left[\left[K_{i j}^{m}\left\{M_{q_{1} q_{2}, j}^{(2)}+\frac{1}{2}\left(\delta_{j q_{2}} M_{q_{1}}^{(1)}+\delta_{j q_{1}} M_{q_{2}}^{(1)}\right)\right\} n_{i}\right]\right]\right|_{\boldsymbol{\xi} \in \Sigma_{1}}=0 .}
\end{aligned}
$$

Perturbation function $\tilde{M}_{p q_{1}}^{(2,1)}$ is provided by the following cell problem and relative interface conditions

$$
\begin{aligned}
& \left(K_{i j}^{m} \tilde{M}_{p q_{1}, j}^{(2,1)}\right)_{, i}-\left(\alpha_{i j}^{m} N_{i p q_{1}, j}^{(1)}+\alpha_{p q_{1}}^{m}\right)=-\left\langle\alpha_{i j}^{m} N_{i p q_{1}, j}^{(1)}+\alpha_{p q_{1}}^{m}\right\rangle, \\
& {\left.\left[\left[\tilde{M}_{p q_{1}}^{(2,1)}\right]\right]\right|_{\boldsymbol{\xi} \in \Sigma_{1}}=0,} \\
& {\left.\left[\left[K_{i j}^{m} \tilde{M}_{p q_{1}, j}^{(2,1)} n_{i}\right]\right]\right|_{\boldsymbol{\xi} \in \Sigma_{1}}=0 .}
\end{aligned}
$$

The third cell problem and its interface conditions have the following expressions in terms of perturbation function $M^{(2,1)}$

$$
\begin{aligned}
& \left(K_{i j}^{m} M_{, j}^{(2,1)}\right)_{, i}-\left(\alpha_{i j}^{m} \tilde{N}_{i, j}^{(1)}+p^{m}\right)=-\left\langle\alpha_{i j}^{m} \tilde{N}_{i, j}^{(1)}+p^{m}\right\rangle, \\
& {\left.\left[\left[M^{(2,1)}\right]\right]\right|_{\boldsymbol{\xi} \in \Sigma_{1}},} \\
& {\left.\left[\left[K_{i j}^{m} M_{, j}^{2,1} n_{i}\right]\right]\right|_{\boldsymbol{\xi} \in \Sigma_{1}}=0 .}
\end{aligned}
$$

Finally, the fourth cell problem and its interface conditions at the order $\varepsilon^{0}$ read

$$
\left(K_{i j}^{m} \hat{M}_{, j}^{(2,1)}\right)_{, i}-\left(\alpha_{i j}^{m} \hat{N}_{i, j}^{(1)}+\psi^{m}\right)=-\left\langle\alpha_{i j}^{m} \hat{N}_{i, j}^{(1)}+\psi^{m}\right\rangle,
$$




$$
\begin{aligned}
& {\left.\left[\left[\hat{M}^{(2,1)}\right]\right]\right|_{\boldsymbol{\xi} \in \Sigma_{1}}=0,} \\
& {\left.\left[\left[K_{i j}^{m} \hat{M}_{, j}^{(2,1)} n_{i}\right]\right]\right|_{\boldsymbol{\xi} \in \Sigma_{1}}=0,}
\end{aligned}
$$

in terms of $\hat{M}^{(2,1)}$.

\section{Mass diffusion cell problems}

Analogously to what done for the thermal problem, at the order $\varepsilon^{-1}$, from equation $(30)$ and taking into account solution (33), one obtains the following cell problem and its interface conditions in terms of perturbation function $W_{q_{1}}^{(1)}$

$$
\begin{aligned}
& \left(D_{i j}^{m} W_{q_{1}, j}^{(1)}\right)_{, i}+D_{i q_{1}, i}^{m}=0, \\
& {\left.\left[\left[W_{q_{1}}^{(1)}\right]\right]\right|_{\boldsymbol{\xi} \in \Sigma_{1}}=0,} \\
& {\left.\left[\left[D_{i j}^{m}\left(W_{q_{1}, j}^{(1)}+\delta_{q_{1} j}\right) n_{i}\right]\right]\right|_{\boldsymbol{\xi} \in \Sigma_{1}}=0 .}
\end{aligned}
$$

At the order $\varepsilon^{0}$, the following four cell problems arise, once first-order perturbation function $W_{q_{1}}^{(1)}$ is computed as solutioon of 59. The first cell problem provides second order perturbation function $W_{q_{1} q_{2}}^{(2)}$ and it is expressed in the following way, symmetrized with respect to indices $q_{1}$ and $q_{2}$, together with its interface conditions

$$
\begin{aligned}
& \left(D_{i j}^{m} W_{q_{1} q_{2}, j}^{(2)}\right)_{, i}+\frac{1}{2}\left[\left(D_{i q_{2}}^{m} W_{q_{1}}^{(1)}\right)_{, i}+D_{q_{1} q_{2}}^{m}+D_{q_{2} i}^{m} W_{q_{1}, i}^{(1)}+\right. \\
& \left.\left(D_{i q_{1}}^{m} W_{q_{2}}^{(1)}\right)_{, i}+D_{q_{2} q_{1}}^{m}+D_{q_{1} i}^{m} W_{q_{2}, i}^{(1)}\right]=\frac{1}{2}\left\langle D_{q_{1} q_{2}}^{m}+D_{q_{2} i}^{m} W_{q_{1}, i}^{(1)}+D_{q_{2} q_{1}}^{m}+D_{q_{1} i}^{m} W_{q_{2}, i}^{(1)}\right\rangle, \\
& {\left.\left[\left[W_{q_{1} q_{2}}^{(2)}\right]\right]\right|_{\boldsymbol{\xi} \in \Sigma_{1}}=0} \\
& {\left.\left[\left[D_{i j}^{m}\left\{W_{q_{1} q_{2}, j}^{(2)}+\frac{1}{2}\left(\delta_{j q_{2}} W_{q_{1}}^{(1)}+\delta_{j q_{1}} W_{q_{2}}^{(1)}\right)\right\} n_{i}\right]\right]\right|_{\boldsymbol{\xi} \in \Sigma_{1}}=0 .}
\end{aligned}
$$

The second cell problem and its interface conditions have the following form in terms of $\tilde{W}_{p q_{1}}^{(2,1)}$

$$
\begin{aligned}
& \left(D_{i j}^{m} \tilde{W}_{p q_{1}, j}^{(2,1)}\right)_{, i}-\left(\beta_{i j}^{m} N_{i p q_{1}, j}^{(1)}+\beta_{p q_{1}}^{m}\right)=-\left\langle\beta_{i j}^{m} N_{i p q_{1}, j}^{(1)}+\beta_{p q_{1}}^{m}\right\rangle, \\
& {\left.\left[\left[\tilde{W}_{p q_{1}}^{(2,1)}\right]\right]\right|_{\boldsymbol{\xi} \in \Sigma_{1}}=0,} \\
& {\left.\left[\left[D_{i j}^{m} \tilde{W}_{p q_{1}, j}^{(2,1)} n_{i}\right]\right]\right|_{\boldsymbol{\xi} \in \Sigma_{1}}=0 .}
\end{aligned}
$$

Second order perturbation function $W^{(2,1)}$ is provided by the resolution of the following cell problem with relative interface conditions

$$
\begin{aligned}
& \left(D_{i j}^{m} W_{, j}^{(2,1)}\right)_{, i}-\left(\beta_{i j}^{m} \hat{N}_{i, j}^{(1)}+q^{m}\right)=-\left\langle\beta_{i j}^{m} \hat{N}_{i, j}^{(1)}+q^{m}\right\rangle, \\
& {\left.\left[\left[W^{(2,1)}\right]\right]\right|_{\boldsymbol{\xi} \in \Sigma_{1}}=0,} \\
& {\left.\left[\left[D_{i j}^{m} W_{, j}^{(2,1)} n_{i}\right]\right]\right|_{\boldsymbol{\xi} \in \Sigma_{1}}=0 .}
\end{aligned}
$$

Finally, the last cell problem at the order $\varepsilon^{0}$ is expressed in the following way, together with interface conditions, in terms of perturbation function $\hat{W}^{(2,1)}$

$$
\begin{aligned}
& \left(D_{i j}^{m} \hat{W}_{, j}^{(2,1)}\right)_{, i}-\left(\beta_{i j}^{m} \tilde{N}_{i, j}^{(1)}+\psi^{m}\right)=-\left\langle\beta_{i j}^{m} \tilde{N}_{i, j}^{(1)}+\psi^{m}\right\rangle, \\
& {\left.\left[\left[\hat{W}^{(2,1)}\right]\right]\right|_{\boldsymbol{\xi} \in \Sigma_{1}}=0,} \\
& {\left.\left[\left[D_{i j}^{m} \hat{W}_{, j}^{(2,1)} n_{i}\right]\right]\right|_{\boldsymbol{\xi} \in \Sigma_{1}}=0 .}
\end{aligned}
$$




\section{Down-scaling and up-scaling relations}

When perturbation functions are known from the resolution of relative cell problems at the different orders of $\varepsilon$ as detailed in Section 3 , from equations (6a)- $6 \mathrm{cc}$ microscopic fields $\mathbf{u}(\mathbf{x}, \mathbf{x} / \varepsilon, t), \theta(\mathbf{x}, \mathbf{x} / \varepsilon, t)$ and $\eta(\mathbf{x}, \mathbf{x} / \varepsilon, t)$ are expressed as asymptotic expansions in powers of micro characteristic size $\varepsilon$ in terms of such $\mathcal{Q}$-periodic perturbation functions and in terms of macrofields $\mathbf{U}(\mathbf{x}, t), \Theta(\mathbf{x}, t)$ and $\Upsilon(\mathbf{x}, t)$ and their gradients. Considering the form of solutions $(25),(29),(33)$ at the order $\varepsilon^{-1}$ and $(37),(41),(45)$ at the order $\varepsilon^{0}$, the following down-scaling relations are obtained for the three microfields

$$
\begin{aligned}
& u_{k}\left(\mathbf{x}, \frac{\mathbf{x}}{\varepsilon}, t\right)=\left[U_{k}(\mathbf{x}, t)+\varepsilon\left(N_{k p q_{1}}^{(1)}(\boldsymbol{\xi}) \frac{\partial U_{p}(\mathbf{x}, t)}{\partial x_{q_{1}}}+\tilde{N}_{k}^{(1)}(\boldsymbol{\xi}) \Theta(\mathbf{x}, t)+\hat{N}_{k}^{(1)}(\boldsymbol{\xi}) \Upsilon(\mathbf{x}, t)\right)+\right. \\
& \left.+\varepsilon^{2}\left(N_{k p q_{1} q_{2}}^{(2)}(\boldsymbol{\xi}) \frac{\partial^{2} U_{p}(\mathbf{x}, t)}{\partial x_{q_{1}} \partial x_{q_{2}}}+\tilde{N}_{k q_{1}}^{(2)}(\boldsymbol{\xi}) \frac{\partial \Theta(\mathbf{x}, t)}{\partial x_{q_{1}}}+\hat{N}_{k q_{1}}^{(2)}(\boldsymbol{\xi}) \frac{\partial \Upsilon(\mathbf{x}, t)}{\partial x_{q_{1}}}+N_{k p}^{(2,2)}(\boldsymbol{\xi}) \frac{\partial^{2} U_{p}(\mathbf{x}, t)}{\partial t^{2}}\right)+O\left(\varepsilon^{3}\right)\right]\left.\right|_{\boldsymbol{\xi}=\frac{\mathbf{x}}{\varepsilon}}, \\
& \theta(64 \mathrm{a}) \\
& \left.\left.+M^{(2,1)}(\boldsymbol{\mathbf { x }}) \frac{\partial \Theta(\mathbf{x}, t)}{\partial t}+\hat{M}^{(2,1)}(\boldsymbol{\xi}) \frac{\partial \Upsilon(\mathbf{x}, t)}{\partial t}\right)+O\left(\varepsilon^{3}\right)\right]\left.\right|_{\boldsymbol{\xi}=\frac{\mathbf{x}}{\varepsilon}}, \\
& \eta\left(\mathbf{x}, \frac{\mathbf{x}}{\varepsilon}, t\right)=\left[\Upsilon(\mathbf{x}, t)+\varepsilon M_{q_{1}}^{(1)}(\boldsymbol{\xi}) \frac{\partial \Theta(\mathbf{x}, t)}{\partial x_{q_{1}}}+\varepsilon^{2}\left(M_{q_{1} q_{2}}^{(2)}(\boldsymbol{\xi}) \frac{\partial^{2} \Theta(\mathbf{x}, t)}{\partial x_{q_{1}} \partial x_{q_{2}}}+\tilde{M}_{p q_{1}}^{(2,1)}(\boldsymbol{\xi}) \frac{\partial^{2} U_{p}(\mathbf{x}, t)}{\partial x_{q_{1}} \partial t}+\right.\right. \\
& \left.+W^{(2,1)}(\boldsymbol{\xi}) \frac{\partial \Upsilon(\mathbf{x}, t)}{\partial t}+\hat{W}_{q_{1}}^{(1)}(\boldsymbol{\xi}) \frac{\partial \Upsilon(\mathbf{x}, t)}{\partial x_{q_{1}}}+\varepsilon^{2}\left(W_{q_{1} q_{2}}^{(2)}(\boldsymbol{\xi}) \frac{\partial^{2} \Upsilon(\mathbf{x}, t)}{\partial x_{q_{1}} \partial x_{q_{2}}}+\tilde{W}_{p q_{1}}^{(2,1)}(\boldsymbol{\xi}) \frac{\partial \Theta(\mathbf{x}, t)}{\partial t}\right)+O\left(\varepsilon^{3}\right)\right]\left.\right|_{\boldsymbol{\xi}=\frac{\mathbf{x}}{\varepsilon}} \cdot
\end{aligned}
$$

In equations (64) microstructural heterogeneities are taken into account by the $\mathcal{Q}$-periodic perturbation functions, which depend exclusively upon the fast variable $\boldsymbol{\xi}$, while the $\mathcal{L}$-periodic macrofields depend solely upon the slow variable $\mathbf{x}$. Up-scaling relations are the ones that provide macroscopic fields $\mathbf{U}(\mathbf{x}, t), \Theta(\mathbf{x}, t)$ and $\Upsilon(\mathbf{x}, t)$ in terms of the corresponding microscopic quantities. In particular, macro fields are expressed as mean values of micro fields over the unit cell $\mathcal{Q}$

$$
\begin{aligned}
U_{k}(\mathbf{x}, t) & \doteq\left\langle u_{k}\left(\mathbf{x}, \frac{\mathbf{x}}{\varepsilon}+\boldsymbol{\zeta}, t\right)\right\rangle_{\boldsymbol{\zeta}}, \\
\Theta(\mathbf{x}, t) & \doteq\left\langle\theta\left(\mathbf{x}, \frac{\mathbf{x}}{\varepsilon}+\boldsymbol{\zeta}, t\right)\right\rangle_{\boldsymbol{\zeta}} \\
\Upsilon(\mathbf{x}, t) & \doteq\left\langle\eta\left(\mathbf{x}, \frac{\mathbf{x}}{\varepsilon}+\boldsymbol{\zeta}, t\right)\right\rangle_{\boldsymbol{\zeta}}
\end{aligned}
$$

where variable $\zeta \in \mathcal{Q}$ is a translation variable such that $\varepsilon \zeta \in \mathcal{A}$ describes the translation of the body with respect to $\mathcal{L}$-periodic source terms, thus removing rapid fluctuations of coefficients (Smyshlyaev and Cherednichenko, 2000, Bacigalupo, 2014). Invariance property

$$
\langle g(\boldsymbol{\xi}+\boldsymbol{\zeta})\rangle_{\boldsymbol{\zeta}}=\frac{1}{\delta} \int_{\mathcal{Q}} g(\boldsymbol{\xi}+\boldsymbol{\zeta}) d \boldsymbol{\zeta}=\frac{1}{\delta} \int_{\mathcal{Q}} g(\boldsymbol{\xi}+\boldsymbol{\zeta}) d \boldsymbol{\xi}
$$

is proved to hold for all functions with $\mathcal{Q}$-periodicity.

\section{Overall constitutive tensors and field equations of the first order homogenized thermo-diffusive medium}

Average field equations of infinite order are determined from the substitution of down-scaling relations (64) into local balance equations $(3 \mathrm{a})-(3 \mathrm{c})$ and ordering at the different orders of $\varepsilon$. They are expressed in the following form

$$
n_{i p q_{1} q_{2}}^{(2)} \frac{\partial^{2} U_{p}}{\partial x_{q_{1}} \partial x_{q_{2}}}-\tilde{n}_{i q_{1}}^{(2)} \frac{\partial \Theta}{\partial x_{q_{1}}}-\hat{n}_{i q_{1}}^{(2)} \frac{\partial \Upsilon}{\partial x_{q_{1}}}-n^{(2,2)} \frac{\partial^{2} U_{i}}{\partial t^{2}}+O(\varepsilon)+b_{i}(\mathbf{x}, t)=0,
$$




$$
\begin{aligned}
& m_{q_{1} q_{2}}^{(2)} \frac{\partial^{2} \Theta}{\partial x_{q_{1}} \partial x_{q_{2}}}-\tilde{m}_{p q_{1}}^{(2,1)} \frac{\partial^{2} U_{p}}{\partial x_{q_{1}} \partial t}-m^{(2,1)} \frac{\partial \Theta}{\partial t}-\hat{m}^{(2,1)} \frac{\partial \Upsilon}{\partial t}+O(\varepsilon)+r(\mathbf{x}, t)=0, \\
& w_{q_{1} q_{2}}^{(2)} \frac{\partial^{2} \Upsilon}{\partial x_{q_{1}} \partial x_{q_{2}}}-\tilde{w}_{p q_{1}}^{(2,1)} \frac{\partial^{2} U_{p}}{\partial x_{q_{1}} \partial t}-w^{(2,1)} \frac{\partial \Upsilon}{\partial t}-\hat{w}^{(2,1)} \frac{\partial \Theta}{\partial t}+O(\varepsilon)+s(\mathbf{x}, t)=0 .
\end{aligned}
$$

Coefficients of macro fields gradients in expressions (67) are defined as mean values over $\mathcal{Q}$ of linear combinations of perturbation functions and microscopic constitutive tensors components. They are the known terms of the corresponding cell problems and, at the order $\varepsilon^{0}$, they read

$$
\begin{aligned}
& n_{i p q_{1} q_{2}}^{(2)}=\frac{1}{2}\left\langle C_{i q_{2} p q_{1}}^{m}+C_{i q_{2} k l}^{m} N_{k p q_{1}, l}^{(1)}+C_{i q_{1} p q_{2}}^{m}+C_{i q_{1} k l}^{m} N_{k p q_{2}, l}^{(1)}\right\rangle, \\
& \tilde{n}_{i q_{1}}^{(2)}=\left\langle\alpha_{i q_{1}}^{m}-C_{i q_{1} k j}^{m} \tilde{N}_{k, j}^{(1)}\right\rangle, \\
& \hat{n}_{i q_{1}}^{(2)}=\left\langle\beta_{i q_{1}}^{m}-C_{i q_{1} k j}^{m} \hat{N}_{k, j}^{(1)}\right\rangle, \\
& n^{(2,2)}=\left\langle\rho^{m}\right\rangle, \\
& m_{q_{1} q_{2}}^{(2)}=\frac{1}{2}\left\langle K_{q_{1} q_{2}}^{m}+K_{q_{2} j} M_{q_{1}, j}^{(1)}+K_{q_{2} q_{1}}^{m}+K_{q_{1} j} M_{q_{2}, j}^{(1)}\right\rangle, \\
& \tilde{m}_{p q_{1}}^{(2,1)}=\left\langle\alpha_{p q_{1}}^{m}+\alpha_{i q_{2}}^{m} N_{i p q_{1}, q_{2}}^{(1)}\right\rangle, \\
& m^{(2,1)}=\left\langle p^{m}+\alpha_{q_{1} q_{2}}^{m} \tilde{N}_{q_{1}, q_{2}}^{(1)}\right\rangle, \\
& \hat{m}^{(2,1)}=\left\langle\psi^{m}+\alpha_{q_{1} q_{2}}^{m} \hat{N}_{q_{1}, q_{2}}^{(1)}\right\rangle, \\
& w_{q_{1} q_{2}}^{(2)}=\frac{1}{2}\left\langle D_{q_{1} q_{2}}^{m}+D_{q_{2} j} W_{q_{1}, j}^{(1)}+D_{q_{2} q_{1}}^{m}+D_{q_{1} j} W_{q_{2}, j}^{(1)}\right\rangle, \\
& \tilde{w}_{p q_{1}}^{(2,1)}=\left\langle\beta_{p q_{1}}^{m}+\beta_{i q_{2}}^{m} N_{i p q_{1}, q_{2}}^{(1)}\right\rangle, \\
& w^{(2,1)}=\left\langle q^{m}+\beta_{q_{1} q_{2}}^{m} \hat{N}_{q_{1}, q_{2}}^{(1)}\right\rangle, \\
& \hat{w}^{(2,1)}=\left\langle\psi^{m}+\beta_{q_{1} q_{2}}^{m} \tilde{N}_{q_{1}, q_{2}}^{(1)}\right\rangle .
\end{aligned}
$$

If one performs the following asymptotic expansions of the macro fields $\mathbf{U}(\mathbf{x}, t), \Theta(\mathbf{x}, t)$ and $\Upsilon(\mathbf{x}, t)$ in powers of characteristic length $\varepsilon$

$$
\begin{aligned}
U_{k}(\mathbf{x}, t) & =\sum_{j=0}^{+\infty} \varepsilon^{j} U_{k}^{(j)}(\mathbf{x}, t), \\
\Theta(\mathbf{x}, t) & =\sum_{j=0}^{+\infty} \varepsilon^{j} \Theta^{(j)}(\mathbf{x}, t), \\
\Upsilon(\mathbf{x}, t) & =\sum_{j=0}^{+\infty} \varepsilon^{j} \Upsilon^{(j)}(\mathbf{x}, t),
\end{aligned}
$$

a formal solution of the average field equations of infinite order (67) can be obtained. In particular, substituting expansions (69) into (67), and reordering at the different orders of $\varepsilon$, one obtains the following three sets of recursive differential problems in terms of the macroscopic fields. Equation 67a becomes

$$
\begin{aligned}
& n_{i p q_{1} q_{2}}^{(2)}\left(\frac{\partial^{2} U_{p}^{(0)}}{\partial x_{q_{1}} \partial x_{q_{2}}}+\varepsilon \frac{\partial^{2} U_{p}^{(1)}}{\partial x_{q_{1}} \partial x_{q_{2}}}+\varepsilon^{2} \frac{\partial^{2} U_{p}^{(2)}}{\partial x_{q_{1}} \partial x_{q_{2}}}+\ldots\right)+\varepsilon n_{i p q_{1} \ldots q_{3}}^{(3)}\left(\frac{\partial^{3} U_{p}^{(0)}}{\partial x_{q_{1}} \ldots \partial x_{q_{3}}}+\varepsilon \frac{\partial^{3} U_{p}^{(1)}}{\partial x_{q_{1}} \ldots \partial x_{q_{3}}}+\right. \\
& \left.+\varepsilon^{2} \frac{\partial^{3} U_{p}^{(2)}}{\partial x_{q_{1}} \ldots \partial x_{q_{3}}}+\ldots\right)+\varepsilon^{2} n_{i p q_{1} \ldots q_{4}}^{(4)}\left(\frac{\partial^{4} U_{p}^{(0)}}{\partial x_{q_{1}} \ldots \partial x_{q_{4}}}+\varepsilon \frac{\partial^{4} U_{p}^{(1)}}{\partial x_{q_{1}} \ldots \partial x_{q_{4}}}+\varepsilon^{2} \frac{\partial^{4} U_{p}^{(2)}}{\partial x_{q_{1}} \ldots \partial x_{q_{4}}}+\ldots\right)+ \\
& -\tilde{n}_{i q_{1}}^{(2)}\left(\frac{\partial \Theta^{(0)}}{\partial x_{q_{1}}}+\varepsilon \frac{\partial \Theta^{(1)}}{\partial x_{q_{1}}}+\varepsilon^{2} \frac{\partial \Theta^{(2)}}{\partial x_{q_{1}}}+\ldots\right)-\varepsilon \tilde{n}_{i q_{1} q_{2}}^{(3)}\left(\frac{\partial^{2} \Theta^{(0)}}{\partial x_{q_{1}} \partial x_{q_{2}}}+\varepsilon \frac{\partial^{2} \Theta^{(1)}}{\partial x_{q_{1}} \partial x_{q_{2}}}+\right.
\end{aligned}
$$




$$
\begin{aligned}
& \left.+\varepsilon^{2} \frac{\partial^{2} \Theta^{(2)}}{\partial x_{q_{1}} \partial x_{q_{2}}}+\ldots\right)-\varepsilon^{2} \tilde{n}_{i q_{1} \ldots q_{3}}^{(4)}\left(\frac{\partial^{3} \Theta^{(0)}}{\partial x_{q_{1}} \ldots \partial x_{q_{3}}}+\varepsilon \frac{\partial^{3} \Theta^{(1)}}{\partial x_{q_{1}} \ldots \partial x_{q_{3}}}+\varepsilon^{2} \frac{\partial^{3} \Theta^{(2)}}{\partial x_{q_{1}} \ldots \partial x_{q_{3}}}+\ldots\right)+ \\
& -\hat{n}_{i q_{1}}^{(2)}\left(\frac{\partial \Upsilon^{(0)}}{\partial x_{q_{1}}}+\varepsilon \frac{\partial \Upsilon^{(1)}}{\partial x_{q_{1}}}+\varepsilon^{2} \frac{\partial \Upsilon^{(2)}}{\partial x_{q_{1}}}+\ldots\right)-\varepsilon \hat{n}_{i q_{1} q_{2}}^{(3)}\left(\frac{\partial^{2} \Upsilon^{(0)}}{\partial x_{q_{1}} \partial x_{q_{2}}}+\varepsilon \frac{\partial^{2} \Upsilon^{(1)}}{\partial x_{q_{1}} \partial x_{q_{2}}}+\right. \\
& \left.+\varepsilon^{2} \frac{\partial^{2} \Upsilon^{(2)}}{\partial x_{q_{1}} \partial x_{q_{2}}}+\ldots\right)-\varepsilon^{2} \hat{n}_{i q_{1} \ldots q_{3}}^{(4)}\left(\frac{\partial^{3} \Upsilon^{(0)}}{\partial x_{q_{1}} \ldots \partial x_{q_{3}}}+\varepsilon \frac{\partial^{3} \Upsilon^{(1)}}{\partial x_{q_{1}} \ldots \partial x_{q_{3}}}+\varepsilon^{2} \frac{\partial^{3} \Upsilon^{(2)}}{\partial x_{q_{1}} \ldots \partial x_{q_{3}}}+\ldots\right)+ \\
& -n_{i}^{(2,2)}\left(\frac{\partial^{2} U_{i}^{(0)}}{\partial t^{2}}+\varepsilon \frac{\partial^{2} U_{i}^{(1)}}{\partial t^{2}}+\varepsilon^{2} \frac{\partial^{2} U_{i}^{(2)}}{\partial t^{2}}+\ldots\right)-\varepsilon n_{i q_{1}}^{(3,2)}\left(\frac{\partial^{3} U_{i}^{(0)}}{\partial x_{q_{1}} \partial t^{2}}+\varepsilon \frac{\partial^{3} U_{i}^{(1)}}{\partial x_{q_{1}} \partial t^{2}}+\right. \\
& \left.+\varepsilon^{2} \frac{\partial^{3} U_{i}^{(2)}}{\partial x_{q_{1}} \partial t^{2}}+\ldots\right)-\varepsilon^{2} n_{i q_{1} q_{2}}^{(4,2)}\left(\frac{\partial^{4} U_{i}^{(0)}}{\partial x_{q_{1}} \partial x_{q_{2}} \partial t^{2}}+\varepsilon \frac{\partial^{4} U_{i}^{(1)}}{\partial x_{q_{1}} \partial x_{q_{2}} \partial t^{2}}+\varepsilon^{2} \frac{\partial^{4} U_{i}^{(2)}}{\partial x_{q_{1}} \partial x_{q_{2}} \partial t^{2}}+\ldots\right)+\ldots+b_{i}(\mathbf{x}, t)=0 .
\end{aligned}
$$

From equation $67 \mathrm{~b}$ one obtains

$$
\begin{aligned}
& m_{q_{1} q_{2}}^{(2)}\left(\frac{\partial^{2} \Theta^{(0)}}{\partial x_{q_{1}} \partial x_{q_{2}}}+\varepsilon \frac{\partial^{2} \Theta^{(1)}}{\partial x_{q_{1}} \partial x_{q_{2}}}+\varepsilon^{2} \frac{\partial^{2} \Theta^{(2)}}{\partial x_{q_{1}} \partial x_{q_{2}}}+\ldots\right)+\varepsilon m_{q_{1} \ldots q_{3}}^{(3)}\left(\frac{\partial^{3} \Theta^{(0)}}{\partial x_{q_{1}} \ldots \partial x_{q_{3}}}+\varepsilon \frac{\partial^{3} \Theta^{(1)}}{\partial x_{q_{1}} \ldots \partial x_{q_{3}}}+\right. \\
& \left.+\varepsilon^{2} \frac{\partial^{3} \Theta^{(2)}}{\partial x_{q_{1}} \ldots \partial x_{q_{3}}}+\ldots\right)+\varepsilon^{2} m_{q_{1} \ldots q_{4}}^{(4)}\left(\frac{\partial^{4} \Theta^{(0)}}{\partial x_{q_{1}} \ldots \partial x_{q_{4}}}+\varepsilon \frac{\partial^{4} \Theta^{(1)}}{\partial x_{q_{1} \ldots \partial x_{q_{4}}}}+\varepsilon^{2} \frac{\partial^{4} \Theta^{(2)}}{\partial x_{q_{1}} \ldots \partial x_{q_{4}}}+\ldots\right)+ \\
& -\tilde{m}_{p_{1}}^{(2,1)}\left(\frac{\partial^{2} U_{p}^{(0)}}{\partial x_{q_{1}} \partial t}+\varepsilon \frac{\partial^{2} U_{p}^{(1)}}{\partial x_{q_{1}} \partial t}+\varepsilon^{2} \frac{\partial^{2} U_{p}^{(2)}}{\partial x_{q_{1}} \partial t}+\ldots\right)-\varepsilon \tilde{m}_{p q_{1} q_{2}}^{(3,1)}\left(\frac{\partial^{3} U_{p}^{(0)}}{\partial x_{q_{1}} \partial x_{q_{2}} \partial t}+\varepsilon \frac{\partial^{3} U_{p}^{(1)}}{\partial x_{q_{1}} \partial x_{q_{2}} \partial t}+\right. \\
& \left.+\varepsilon^{2} \frac{\partial^{3} U_{p}^{(2)}}{\partial x_{q_{1}} \partial x_{q_{2}} \partial t}+\ldots\right)-\varepsilon^{2} \tilde{m}_{p q_{1} \ldots q_{3}}^{(4,1)}\left(\frac{\partial^{4} U_{p}^{(0)}}{\partial x_{q_{1}} \ldots \partial x_{q_{3}} \partial t}+\varepsilon \frac{\partial^{4} U_{p}^{(1)}}{\partial x_{q_{1}} \ldots \partial x_{q_{3}} \partial t}+\varepsilon^{2} \frac{\partial^{4} U_{p}^{(2)}}{\partial x_{q_{1}} \ldots \partial x_{q_{3}} \partial t}+\ldots\right)+ \\
& -m^{(2,1)}\left(\frac{\partial \Theta}{\partial t}+\varepsilon \frac{\partial \Theta^{(1)}}{\partial t}+\varepsilon^{2} \frac{\partial \Theta^{(2)}}{\partial t}+\ldots\right)-\varepsilon m_{q 1}^{(3,1)}\left(\frac{\partial^{2} \Theta^{(0)}}{\partial x_{q_{1}} \partial t}+\varepsilon \frac{\partial^{2} \Theta^{(1)}}{\partial x_{q_{1}} \partial t}+\right. \\
& \left.+\varepsilon^{2} \frac{\partial^{2} \Theta^{(2)}}{\partial x_{q_{1}} \partial t}+\ldots\right)-\varepsilon^{2} m_{q_{1} q_{2}}^{(4,1)}\left(\frac{\partial^{3} \Theta^{(0)}}{\partial x_{q_{1}} \partial x_{q_{2}} \partial t}+\varepsilon \frac{\partial^{3} \Theta^{(1)}}{\partial x_{q_{1}} \partial x_{q_{2}} \partial t}+\varepsilon^{2} \frac{\partial^{3} \Theta^{(2)}}{\partial x_{q_{1}} \partial x_{q_{2}} \partial t}+\ldots\right)+ \\
& -\hat{m}^{(2,1)}\left(\frac{\partial \Upsilon}{\partial t}+\varepsilon \frac{\partial \Upsilon^{(1)}}{\partial t}+\varepsilon^{2} \frac{\partial \Upsilon^{(2)}}{\partial t}+\ldots\right)-\varepsilon \hat{m}_{q_{1}}^{(3,1)}\left(\frac{\partial^{2} \Upsilon^{(0)}}{\partial x_{q_{1}} \partial t}+\varepsilon \frac{\partial^{2} \Upsilon^{(1)}}{\partial x_{q_{1}} \partial t}+\right. \\
& \left.+\varepsilon^{2} \frac{\partial^{2} \Upsilon^{(2)}}{\partial x_{q_{1}} \partial t}+\ldots\right)-\varepsilon^{2} \hat{m}_{q_{1} q_{2}}^{(4,1)}\left(\frac{\partial^{3} \Upsilon^{(0)}}{\partial x_{q_{1}} \partial x_{q_{2}} \partial t}+\varepsilon \frac{\partial^{3} \Upsilon^{(1)}}{\partial x_{q_{1}} \partial x_{q_{2}} \partial t}+\varepsilon^{2} \frac{\partial^{3} \Upsilon^{(2)}}{\partial x_{q_{1}} \partial x_{q_{2}} \partial t}+\ldots\right)+\ldots+r(\mathbf{x}, t)=0 .
\end{aligned}
$$

Finally, equation $67 \mathrm{c}$ reads

$$
\begin{aligned}
& w_{q_{1} q_{2}}^{(2)}\left(\frac{\partial^{2} \Upsilon^{(0)}}{\partial x_{q_{1}} \partial x_{q_{2}}}+\varepsilon \frac{\partial^{2} \Upsilon^{(1)}}{\partial x_{q_{1}} \partial x_{q_{2}}}+\varepsilon^{2} \frac{\partial^{2} \Upsilon^{(2)}}{\partial x_{q_{1}} \partial x_{q_{2}}}+\ldots\right)+\varepsilon m_{q_{1} \ldots q_{3}}^{(3)}\left(\frac{\partial^{3} \Upsilon^{(0)}}{\partial x_{q_{1}} \ldots \partial x_{q_{3}}}+\varepsilon \frac{\partial^{3} \Upsilon^{(1)}}{\partial x_{q_{1}} \ldots \partial x_{q_{3}}}+\right. \\
& \left.+\varepsilon^{2} \frac{\partial^{3} \Upsilon^{(2)}}{\partial x_{q_{1}} \ldots \partial x_{q_{3}}}+\ldots\right)+\varepsilon^{2} m_{q_{1} \ldots q_{4}}^{(4)}\left(\frac{\partial^{4} \Upsilon^{(0)}}{\partial x_{q_{1}} \ldots \partial x_{q_{4}}}+\varepsilon \frac{\partial^{4} \Upsilon^{(1)}}{\partial x_{q_{1}} \ldots \partial x_{q_{4}}}+\varepsilon^{2} \frac{\partial^{4} \Upsilon^{(2)}}{\partial x_{q_{1}} \ldots \partial x_{q_{4}}}+\ldots\right)+ \\
& -\tilde{w}_{p q_{1}}^{(2,1)}\left(\frac{\partial^{2} U_{p}^{(0)}}{\partial x_{q_{1}} \partial t}+\varepsilon \frac{\partial^{2} U_{p}^{(1)}}{\partial x_{q_{1}} \partial t}+\varepsilon^{2} \frac{\partial^{2} U_{p}^{(2)}}{\partial x_{q_{1}} \partial t}+\ldots\right)-\varepsilon \tilde{w}_{p q_{1} q_{2}}^{(3,1)}\left(\frac{\partial^{3} U_{p}^{(0)}}{\partial x_{q_{1}} \partial x_{q_{2}} \partial t}+\varepsilon \frac{\partial^{3} U_{p}^{(1)}}{\partial x_{q_{1}} \partial x_{q_{2}} \partial t}+\right. \\
& \left.+\varepsilon^{2} \frac{\partial^{3} U_{p}^{(2)}}{\partial x_{q_{1}} \partial x_{q_{2}} \partial t}+\ldots\right)-\varepsilon^{2} \tilde{w}_{p q_{1} \ldots q_{3}}^{(4,1)}\left(\frac{\partial^{4} U_{p}^{(0)}}{\partial x_{q_{1}} \ldots \partial x_{q_{3}} \partial t}+\varepsilon \frac{\partial^{4} U_{p}^{(1)}}{\partial x_{q_{1}} \ldots \partial x_{q_{3}} \partial t}+\varepsilon^{2} \frac{\partial^{4} U_{p}^{(2)}}{\partial x_{q_{1}} \ldots \partial x_{q_{3}} \partial t}+\ldots\right)+ \\
& -w^{(2,1)}\left(\frac{\partial \Upsilon^{(0)}}{\partial t}+\varepsilon \frac{\partial \Upsilon^{(1)}}{\partial t}+\varepsilon^{2} \frac{\partial \Upsilon^{(2)}}{\partial t}+\ldots\right)-\varepsilon w_{q_{1}}^{(3,1)}\left(\frac{\partial^{2} \Upsilon^{(0)}}{\partial x_{q_{1}} \partial t}+\varepsilon \frac{\partial^{2} \Upsilon^{(1)}}{\partial x_{q_{1}} \partial t}+\right. \\
& \left.+\varepsilon^{2} \frac{\partial^{2} \Upsilon^{(2)}}{\partial x_{q_{1}} \partial t}+\ldots\right)-\varepsilon^{2} w_{q_{1} q_{2}}^{(4,1)}\left(\frac{\partial^{3} \Upsilon^{(0)}}{\partial x_{q_{1}} \partial x_{q_{2}} \partial t}+\varepsilon \frac{\partial^{3} \Upsilon^{(1)}}{\partial x_{q_{1}} \partial x_{q_{2}} \partial t}+\varepsilon^{2} \frac{\partial^{3} \Upsilon^{(2)}}{\partial x_{q_{1}} \partial x_{q_{2}} \partial t}+\ldots\right)+
\end{aligned}
$$




$$
\begin{aligned}
& -\hat{w}^{(2,1)}\left(\frac{\partial \Theta^{(0)}}{\partial t}+\varepsilon \frac{\partial \Theta^{(1)}}{\partial t}+\varepsilon^{2} \frac{\partial \Theta^{(2)}}{\partial t}+\ldots\right)-\varepsilon \hat{w}_{q_{1}}^{(3,1)}\left(\frac{\partial^{2} \Theta^{(0)}}{\partial x_{q_{1}} \partial t}+\varepsilon \frac{\partial^{2} \Theta^{(1)}}{\partial x_{q_{1}} \partial t}+\right. \\
& \left.+\varepsilon^{2} \frac{\partial^{2} \Theta^{(2)}}{\partial x_{q_{1}} \partial t}+\ldots\right)-\varepsilon^{2} \hat{w}_{q_{1} q_{2}}^{(4,1)}\left(\frac{\partial^{3} \Theta^{(0)}}{\partial x_{q_{1}} \partial x_{q_{2}} \partial t}+\varepsilon \frac{\partial^{3} \Theta^{(1)}}{\partial x_{q_{1}} \partial x_{q_{2}} \partial t}+\varepsilon^{2} \frac{\partial^{3} \Theta^{(2)}}{\partial x_{q_{1}} \partial x_{q_{2}} \partial t}+\ldots\right)+\ldots+s(\mathbf{x}, t)=0 .
\end{aligned}
$$

Truncating at the order $\varepsilon^{0}$, from equation 70 the following macro differential problem is derived

$$
n_{i p q_{1} q_{2}}^{(2)} \frac{\partial^{2} U_{p}^{(0)}}{\partial x_{q_{1}} \partial x_{q_{2}}}-\tilde{n}_{i q_{1}}^{(2)} \frac{\partial \Theta^{(0)}}{\partial x_{q_{1}}}-\hat{n}_{i q_{1}}^{(2)} \frac{\partial \Upsilon^{(0)}}{\partial x_{q_{1}}}-n^{(2,2)} \frac{\partial^{2} U_{i}^{(0)}}{\partial t^{2}}+b_{i}(\mathbf{x}, t)=0 .
$$

Analogously, macro differential problem obtained truncating equation 71 at the order $\varepsilon^{0}$ has the form

$$
m_{q_{1} q_{2}}^{(2)} \frac{\partial^{2} \Theta^{(0)}}{\partial x_{q_{1}} \partial x_{q_{2}}}-\tilde{m}_{p q_{1}}^{(2,1)} \frac{\partial^{2} U_{p}^{(0)}}{\partial x_{q_{1}} \partial t}-m^{(2,1)} \frac{\partial \Theta^{(0)}}{\partial t}-\hat{m}^{(2,1)} \frac{\partial \Upsilon^{(0)}}{\partial t}+r(\mathbf{x}, t)=0 .
$$

Third macro problem from equation 72 reads

$$
w_{q_{1} q_{2}}^{(2)} \frac{\partial^{2} \Upsilon^{(0)}}{\partial x_{q_{1}} \partial x_{q_{2}}}-\tilde{w}_{p q_{1}}^{(2,1)} \frac{\partial^{2} U_{p}^{(0)}}{\partial x_{q_{1}} \partial t}-w^{(2,1)} \frac{\partial \Upsilon^{(0)}}{\partial t}-\hat{w}^{(2,1)} \frac{\partial \Theta^{(0)}}{\partial t}+s(\mathbf{x}, t)=0 .
$$

The following normalization conditions

$$
\frac{1}{\delta L^{2}} \int_{\mathcal{L}} U_{p}^{(m)}(\mathbf{x}, t) d \mathbf{x}=0, \frac{1}{\delta L^{2}} \int_{\mathcal{L}} \Theta^{(m)}(\mathbf{x}, t) d \mathbf{x}=0, \frac{1}{\delta L^{2}} \int_{\mathcal{L}} \Upsilon^{(m)}(\mathbf{x}, t) d \mathbf{x}=0,
$$

are demanded to be satisfied by macro fields $\mathbf{U}(\mathbf{x}, t)^{(m)}, \Theta^{(m)}(\mathbf{x}, t)$ and $\Upsilon^{(m)}(\mathbf{x}, t)$, in the case of $\mathcal{L}$-periodic source terms, for each $m \in \mathbb{Z}$. In this case, macro fields result to be $\mathcal{L}$-periodic, too. If source terms are not $\mathcal{L}$-periodic, normalization conditions $\sqrt{76}$ need to be substituted by appropriate boundary conditions to compute the macro fields. In fact, $\mathcal{L}$-periodicity is not a mandatory requirement for source terms. These lasts are only required to show a variability much greater than the characteristic microstructural length $\varepsilon$ in order to preserve the separation of scales. In order to derive governing field equations for the firstorder homogenized continuum, zeroth order differential problems (73)- 75 need to be expressed in terms of components $C_{i q_{1} p q_{2}}, \alpha_{i q_{1}}, \beta_{i q_{1}}, K_{q_{1} q_{2}}, D_{q_{1} q_{2}}$ of overall constitutive tensors, in terms of overall thermo-diffusive coupling constant $\psi$ and overall inertial terms $\rho, p$ and $q$. Relations between components of the relative overall constitutive tensors $\mathfrak{C}, \mathbf{K}$, and $\mathbf{D}$ and the ones of tensors $\mathbf{n}^{(2)}, \mathbf{m}^{(2)}$, and $\mathbf{w}^{(2)}$ are detailed in (Fantoni et al. 2017) and read

$$
n_{i p q_{1} q_{2}}^{(2)}=\frac{1}{2}\left(C_{p q_{1} i q_{2}}+C_{p q_{2} i q_{1}}\right), \quad m_{q_{1} q_{2}}^{(2)}=K_{q_{1} q_{2}}, \quad w_{q_{1} q_{2}}^{(2)}=D_{q_{1} q_{2}} .
$$

Symmetries and positive definition of tensors $\mathbf{n}^{(2)}=n_{i p q_{1} q_{1}}^{(2)} \mathbf{e}_{i} \otimes \mathbf{e}_{p} \otimes \mathbf{e}_{q_{1}} \otimes \mathbf{e}_{q 2}, \mathbf{m}^{(2)}=m_{q_{1} q_{2}}^{(2)} \mathbf{e}_{q_{1}} \otimes \mathbf{e}_{q_{2}}$, and $\mathbf{w}^{(2)}=w_{q_{1} q_{2}}^{(2)} \mathbf{e}_{q_{1}} \otimes \mathbf{e}_{q_{2}}$ are accurately provided in the above mentioned references, where is proved that such tensors can be expressed as

$$
\begin{aligned}
n_{i p q_{1} q_{2}}^{(2)} & =\frac{1}{2}\left\langle C_{r j k l}^{m}\left(N_{r i q_{2}, j}^{(1)}+\delta_{i r} \delta_{j q_{2}}\right)\left(N_{k p q_{1}, l}^{(1)}+\delta_{p k} \delta_{l q_{1}}\right)+\right. \\
& \left.+C_{r j k l}^{m}\left(N_{r i q_{1}, j}^{(1)}+\delta_{i r} \delta_{j q_{1}}\right)\left(N_{k p q_{2}, l}^{(1)}+\delta_{p k} \delta_{l q_{2}}\right)\right\rangle \\
m_{q_{1} q_{2}}^{(2)} & =K_{q_{1} q_{2}}=\left\langle K_{i j}^{m}\left(M_{q_{2}, i}^{(1)}+\delta_{i q_{2}}\right)\left(M_{q_{1}, j}^{(1)}+\delta_{j q_{1}}\right)\right\rangle \\
w_{q_{1} q_{2}}^{(2)} & =D_{q_{1} q_{2}}=\left\langle D_{i j}^{m}\left(W_{q_{2}, i}^{(1)}+\delta_{i q_{2}}\right)\left(W_{q_{1}, j}^{(1)}+\delta_{j q_{1}}\right)\right\rangle .
\end{aligned}
$$

A comparison between the first of equations $(77)$ and the first of $(78)$ leads to the expression of components of overall elastic tensor $\mathfrak{C}$, namely

$$
C_{p q_{1} i q_{2}}=\left\langle C_{r j k l}^{m}\left(N_{r i q_{2}, j}^{(1)}+\delta_{i r} \delta_{j q_{2}}\right)\left(N_{k p q_{1}, l}^{(1)}+\delta_{p k} \delta_{l q_{1}}\right)\right\rangle .
$$


In Appendix A equalities $\alpha_{p q_{1}}=\tilde{n}_{p q_{1}}^{(2)}=\tilde{m}_{p q_{1}}^{(2,1)}, \beta_{p q_{1}}=\hat{n}_{p q_{1}}^{(2)}=\tilde{w}_{p q_{1}}^{(2,1)}=$ and $\psi=\hat{m}^{(2,1)}=\hat{w}^{(2,1)}$ are proved in detail. Equalities between scalars $n^{(2,2)}=\rho, m^{(2,1)}=p$, and $w^{(2,1)}=q$, involving overall inertial terms, trivially follow. Field equations for the equivalent first-order (Cauchy) thermo-diffusive medium are therefore expressed in the form

$$
\begin{aligned}
& C_{i q_{1} p q_{2}} \frac{\partial^{2} U_{p}(\mathbf{x}, t)}{\partial x_{q_{1}} \partial x_{q_{2}}}-\alpha_{i q_{1}} \frac{\partial \Theta(\mathbf{x}, t)}{\partial x_{q_{1}}}-\beta_{i q_{1}} \frac{\partial \Upsilon(\mathbf{x}, t)}{\partial x_{q_{1}}}-\rho \frac{\partial^{2} U_{i}(\mathbf{x}, t)}{\partial t^{2}}+b_{i}(\mathbf{x}, t)=0, \\
& K_{q_{1} q_{2}} \frac{\partial^{2} \Theta(\mathbf{x}, t)}{\partial x_{q_{1}} \partial x_{q_{2}}}-\alpha_{p q_{1}} \frac{\partial^{2} U_{p}(\mathbf{x}, t)}{\partial x_{q_{1}} \partial t}-\psi \frac{\partial \Upsilon(\mathbf{x}, t)}{\partial t}-p \frac{\partial \Theta(\mathbf{x}, t)}{\partial t}+r(\mathbf{x}, t)=0, \\
& D_{q_{1} q_{2}} \frac{\partial^{2} \Upsilon(\mathbf{x}, t)}{\partial x_{q_{1}} \partial x_{q_{2}}}-\beta_{p q_{1}} \frac{\partial^{2} U_{p}(\mathbf{x}, t)}{\partial x_{q_{1}} \partial t}-\psi \frac{\partial \Theta(\mathbf{x}, t)}{\partial t}-q \frac{\partial \Upsilon(\mathbf{x}, t)}{\partial t}+s(\mathbf{x}, t)=0,
\end{aligned}
$$

where macro fields correspond to the zeroth order ones, namely

$$
U_{p}(\mathbf{x}, t) \approx U_{p}^{(0)}(\mathbf{x}, t), \quad \Theta(\mathbf{x}, t) \approx \Theta^{(0)}(\mathbf{x}, t), \Upsilon(\mathbf{x}, t) \approx \Upsilon^{(0)}(\mathbf{x}, t) .
$$

\section{Complex frequency band structure of the equivalent thermo- diffusive medium}

A two-sided Laplace transform of a real valued time dependent function $f: \mathbb{R} \rightarrow \mathbb{R}$ is defined in the following way (Paley and Wiener, 1934)

$$
\mathcal{L}(f(t))=\hat{f}(\omega)=\int_{-\infty}^{+\infty} f(t) e^{-\omega t} d t
$$

with the Laplace argument $\omega \in \mathbb{C}$ and the Laplace transform a complex valued function $\hat{f}: \mathbb{C} \rightarrow \mathbb{C}$. Taking into account the following derivation rule

$$
\mathcal{L}\left(\frac{\partial^{n} f(t)}{\partial t^{n}}\right)=\omega^{n} \hat{f}(\omega)
$$

and performing Laplace transform of field equations 80 , one obtains the following generalized Christoffel equations for the first-order equivalent medium

$$
\begin{aligned}
& C_{i q_{1} p q_{2}} \frac{\partial^{2} \hat{U}_{p}(\mathbf{x}, \omega)}{\partial x_{q_{1}} \partial x_{q_{2}}}-\alpha_{i q_{1}} \frac{\partial \hat{\Theta}(\mathbf{x}, \omega)}{\partial x_{q_{1}}}-\beta_{i q_{1}} \frac{\partial \hat{\Upsilon}(\mathbf{x}, \omega)}{\partial x_{q_{1}}}-\rho \omega^{2} \hat{U}_{i}(\mathbf{x}, \omega)+\hat{b}_{i}(\mathbf{x}, \omega)=0, \\
& K_{q_{1} q_{2}} \frac{\partial^{2} \hat{\Theta}(\mathbf{x}, \omega)}{\partial x_{q_{1}} \partial x_{q_{2}}}-\alpha_{p q_{1}} \omega \frac{\partial \hat{U}_{p}(\mathbf{x}, \omega)}{\partial x_{q_{1}}}-\psi \omega \hat{\Upsilon}(\mathbf{x}, \omega)-p \omega \hat{\Theta}(\mathbf{x}, \omega)+\hat{r}(\mathbf{x}, \omega)=0 \\
& D_{q_{1} q_{2}} \frac{\partial^{2} \hat{\Upsilon}(\mathbf{x}, \omega)}{\partial x_{q_{1}} \partial x_{q_{2}}}-\beta_{p q_{1}} \omega \frac{\partial \hat{U}_{p}(\mathbf{x}, \omega)}{\partial x_{q_{1}}}-\psi \omega \hat{\Theta}(\mathbf{x}, \omega)-q \omega \hat{\Upsilon}(\mathbf{x}, \omega)+\hat{s}(\mathbf{x}, \omega)=0,
\end{aligned}
$$

Fourier transform of a real valued, space varying function $f$ has the following definition (Paley and Wiener. 1934)

$$
\mathcal{F}(f(\mathbf{x}))=\check{f}(\mathbf{k})=\int_{-\infty}^{+\infty} \int_{-\infty}^{+\infty} f(\mathbf{x}) e^{-i \mathbf{k x}} d \mathbf{x}
$$

where Fourier argument $\mathbf{k} \in \mathbb{R}^{2}$ and $i$ is the imaginary unit such that $i^{2}=-1$. Fourier transform of equations (84), bearing in mind derivation rule

$$
\mathcal{F}\left(\frac{\partial^{n} f(\mathbf{x})}{\partial x_{j}^{n}}\right)=\left(i k_{j}\right)^{n} \check{f}(\mathbf{k})
$$

leads to the following equations

$$
-k_{q_{1}} k_{q_{2}} C_{i q_{1} p q_{2}} \check{\hat{U}}_{p}(\mathbf{k}, \omega)-\alpha_{i q_{1}} i k_{q_{1}} \grave{\hat{\Theta}}(\mathbf{k}, \omega)-\beta_{i q_{1}} i k_{q_{1}} \check{\hat{\Upsilon}}(\mathbf{k}, \omega)-\rho \omega^{2} \check{\hat{U}}_{i}(\mathbf{k}, \omega)+\check{\hat{b}}_{i}(\mathbf{k}, \omega)=0
$$




$$
\begin{aligned}
& -k_{q_{1}} k_{q_{2}} K_{q_{1} q_{2}} \check{\hat{\Theta}}(\mathbf{k}, \omega)-\alpha_{p q_{1}} i k_{q_{1}} \omega \check{\hat{U}}_{p}(\mathbf{k}, \omega)-\psi \omega \check{\hat{\Upsilon}}(\mathbf{k}, \omega)-p \omega \check{\hat{\Theta}}(\mathbf{k}, \omega)+\check{\hat{r}}(\mathbf{k}, \omega)=0, \\
& -k_{q_{1}} k_{q_{2}} D_{q_{1} q_{2}} \check{\hat{\Upsilon}}(\mathbf{k}, \omega)-\beta_{p q_{1}} i k_{q_{1}} \omega \check{\hat{U}}_{p}(\mathbf{k}, \omega)-\psi \omega \check{\hat{\Theta}}(\mathbf{k}, \omega)-q \omega \check{\hat{\Upsilon}}(\mathbf{k}, \omega)+\check{\hat{s}}(\mathbf{k}, \omega)=0 .
\end{aligned}
$$

With the aim of studying the propagation of free waves inside the equivalent thermo-diffusive material, source terms are put equal to zero $(\check{\hat{\mathbf{b}}}=\mathbf{0}, \check{\hat{r}}=0, \check{\hat{s}}=0)$ in equations 87 . Waves propagating inside the medium will be damped in time and dispersive, because of the structure of governing field equations 80 . Governing equations in the transformed space and frequency domain (87) can be written in absolute notation as

$$
\begin{aligned}
& \left(\tilde{\mathfrak{C}}(\mathbf{k} \otimes \mathbf{k})+\rho \omega^{2} \mathbf{I}\right) \check{\hat{\mathbf{U}}}(\mathbf{k}, \omega)+i \boldsymbol{\alpha} \mathbf{k} \check{\hat{\Theta}}(\mathbf{k}, \omega)+i \boldsymbol{\beta} \mathbf{k} \check{\hat{\Upsilon}}(\mathbf{k}, \omega)=\mathbf{0}, \\
& (\mathbf{k}:(\mathbf{k} \otimes \mathbf{k})+p \omega) \check{\hat{\Theta}}(\mathbf{k}, \omega)+i \omega(\boldsymbol{\alpha} \mathbf{k}) \cdot \check{\hat{\boldsymbol{U}}}(\mathbf{k}, \omega)+\psi \omega \hat{\hat{\Upsilon}}(\mathbf{k}, \omega)=0, \\
& (\boldsymbol{D}:(\mathbf{k} \otimes \mathbf{k})+q \omega) \check{\hat{\Upsilon}}(\mathbf{k}, \omega)+i \omega(\boldsymbol{\beta} \mathbf{k}) \cdot \dot{\hat{\boldsymbol{U}}}(\mathbf{k}, \omega)+\psi \omega \tilde{\hat{\Theta}}(\mathbf{k}, \omega)=0,
\end{aligned}
$$

and in matrix notation as

$$
\left(\begin{array}{ccc}
\tilde{\mathfrak{C}}(\mathbf{k} \otimes \mathbf{k})+\rho \omega^{2} \mathbf{I} & i \boldsymbol{\alpha} \mathbf{k} & i \boldsymbol{\beta} \mathbf{k} \\
i \omega(\boldsymbol{\alpha} \mathbf{k})^{T} & \mathbf{K}:(\mathbf{k} \otimes \mathbf{k})+p \omega & \psi \omega \\
i \omega(\boldsymbol{\beta} \mathbf{k})^{T} & \psi \omega & \boldsymbol{D}:(\mathbf{k} \otimes \mathbf{k})+q \omega
\end{array}\right)\left(\begin{array}{c}
\check{\hat{\mathbf{U}}} \\
\check{\hat{\Theta}} \\
\check{\hat{\Upsilon}}
\end{array}\right)=\left(\begin{array}{l}
\mathbf{0} \\
0 \\
0
\end{array}\right)
$$

where $\tilde{\mathfrak{C}}=\tilde{C}_{i p q_{1} q_{2}} \mathbf{e}_{i} \otimes \mathbf{e}_{p} \otimes \mathbf{e}_{q_{1}} \otimes \mathbf{e}_{q_{2}}$ and $\tilde{C}_{i p q_{1} q_{2}}=C_{i q_{1} p q_{2}}$ and $\mathbf{I}$ is the identity operator. Equation 89 represents a quadratic generalized eigenvalue problem that can be written in a concise form as

$$
\left(\mathbf{H}_{2} \omega^{2}+\mathbf{H}_{1} \omega+\mathbf{H}_{0}\right) \mathbf{Z}=\mathbf{0},
$$

where $\omega$ corresponds to the generalized eigenvalue and $\mathbf{Z}=(\check{\hat{\mathbf{U}}} \check{\hat{\mathbf{\Theta}}} \check{\hat{\Upsilon}})^{T}$ is the generalized eigenvector. Generalized eigenvalue $\omega$ is the complex angular frequency of the damped wave and its real and imaginary parts describe the damping and the propagation modes of dispersive Bloch waves propagating inside the medium, respectively. Vector $\mathbf{Z}$, which collect the macrofields in the transformed space and frequency domain, is the polarization vector of the damped wave, while $\mathbf{k}=k_{1} \mathbf{e}_{1}+k_{2} \mathbf{e}_{2} \in \mathcal{B}$ represents the wave vector, with $k_{1}$ and $k_{2}$ the wave numbers and $\mathcal{B}=\left[-\pi / d_{1}, \pi / d_{1}\right] \times\left[-\pi / d_{2}, \pi / d_{2}\right]$ the first Brillouin zone associated to periodic cell $\mathcal{A}$. Complex frequencies $\omega$ related to problem $(90)$ are computed as the roots of the characteristic equation

$$
\operatorname{det}(\mathbf{H})=0,
$$

with matrix $\mathbf{H}=\mathbf{H}_{2} \omega^{2}+\mathbf{H}_{1} \omega+\mathbf{H}_{0}$, thus defining the complex frequency band structure of the periodic thermo-diffusive homogenized medium. Complex algebraic operators $\mathbf{H}_{2}, \mathbf{H}_{1}$ and $\mathbf{H}_{0}$ are such that $\mathbf{H}_{2}$ is constant with respect to $\mathbf{k}$, while $\mathbf{H}_{1}$ and $\mathbf{H}_{0}$ quadratically and linearly depend upon $\mathbf{k}$. Consequently, complex angular frequency $\omega$ depends upon $\mathbf{k}$, thus defining the complex dispersion curves characterizing the equivalent medium.

\subsection{Asymptotic approximation of the complex spectrum}

After representing the wave vector components in a polar coordinate system as $k_{1}=r \cos (\phi)$ and $k_{2}=$ $r \sin (\phi)$, with $r=\|\mathbf{k}\|_{2}=\sqrt{k_{1}^{2}+k_{2}^{2}}$ the radial coordinate and $\phi$ the angular coordinate, for a given value of $\phi$, characteristic equation (91) can be written in the form $F(\omega(r), r)=0$. Since the characteristic function $F(\omega(r), r)$ substantially depends upon the $r$ variable, in order to find an explicit solution of the characteristic equation $F(\omega(r), r)=0$, an asymptotic expansion of function $\omega(r)$ is performed in powers of $r$, which essentially acts as a single perturbation parameter. Asymptotic expansion reads

$$
\omega(r)=\omega^{[0]}+\sum_{n \in \mathbb{N}^{*}} \omega^{[n]} r^{n}=\omega^{[0]}+\omega^{[1]} r+\omega^{[2]} r^{2}+\ldots+\omega^{[n]} r^{n}+\ldots
$$

Assuming sufficient regularity for dispersion function $\omega(r)$, expansion $(92)$ locally approximates the exact eigenvalue $\omega$ in the vicinity of the reference point $r=0$. Once multiplied by factorial $n$ !, coefficient $\omega^{[n]}$ of (92) represents the unknown $r$-derivative of order $n$ of the exact, but implicit equation $F(\omega(r), r)=0$. In 
this regard, approximation 92 of dispersion function $\omega(r)$ is tangent to the exact dispersion curve in $r=0$, while, for increasing values of parameter $r$, the accuracy of approximation (92) is expected to diminish. Established the series $(92)$, characteristic function $F(\omega(r), r)$ can be regarded as a composite single variable function $G(r)$ and its Taylor expansion reads

$$
G(r)=G^{[0]}+\sum_{n \in \mathbb{N}^{*}} \frac{G^{[n]}}{n !} r^{n}=G^{[0]}+G^{[1]} r+\frac{G^{[2]}}{2} r^{2}+\ldots+\frac{G^{[n]}}{n !} r^{n}+\ldots
$$

in powers of radial coordinate $r$. Beginning with the generating solution at the order $r^{0}$, which defines the six known eigenvalues $\omega^{[0]}=0$ as solutions of $G^{[0]}(0)=0$, equating to zero each coefficient $G^{[n]}$ at the order $r^{n}$, the approximate characteristic equation $G(r)=0$ results asymptotically satisfied. The procedure gives rise to a chain of $n$-ordered equations called perturbation equations, each one characterized by a single unknown, namely one of the higher order sensitivities $\omega^{[n]}$. Higher order coefficients $G^{[n]}$ of $(93)$ represents the $r$-derivative of order $n$ of function $G(r)$ evaluated at $r=0$, thus requiring the recursive implementation of the chain rule in order to obtain the differentiation of a composite function. Lowest order characteristic polynomials $G^{[1]}$ and $G^{[2]}$ have the form

$$
\begin{aligned}
& r^{1}: \quad G^{[1]}=\omega^{[1]} \frac{\partial F(\omega, r)}{\partial \omega}+\frac{\partial F(\omega, r)}{\partial r} \\
& r^{2}: \quad G^{[2]}=2 \omega^{[2]} \frac{\partial F(\omega, r)}{\partial \omega}+\omega^{[1]} \frac{\partial^{2} F(\omega, r)}{\partial \omega^{2}}+2 \omega^{[1]} \frac{\partial^{2} F(\omega, r)}{\partial \omega \partial r}+\frac{\partial^{2} F(\omega, r)}{\partial r^{2}}
\end{aligned}
$$

where the partial derivatives of function $F$ are evaluated at $\omega=\omega^{[0]}$ and $r=0$. The generalization of the chain rule to higher order derivatives can be found in Bacigalupo and Lepidi (2016), formula (23), where it is expressed in a recursive form of the generic $n_{t h}$ sensitivity. The solution scheme needed to accomplish a fourth order approximation

$$
\omega_{i}(r)=\omega_{i}^{[0]}+\omega_{i}^{[1]} r+\omega_{i}^{[2]} r^{2}+\omega_{i}^{[3]} r^{3}+\omega_{i}^{[4]} r^{4}+O\left(r^{5}\right)
$$

for all the six eigenvalues $(i=1, \ldots, 6)$ is described in table 1 and it is valid for any angular coordinate $\phi$. As evident from table 1 . when sensitivity $\omega_{i}^{[n]}$ has a multiplicity $m>1$, the successive $m-1$ perturbation equations result to be indeterminate and sensitivity $\omega_{i}^{[n+1]}$ is computed as the solution of the next $m_{t h}$ perturbation problem. Perturbative technique described in the present Section allows obtaining a parametric approximation of the complex eigenspectrum of polinomial operator $\mathbf{H}(\omega(r), r)$ at $r=0$, from which the explicit dependence of complex dispersion functions $\omega(r)$ upon the overall constitutive parameters of the homogenized medium is obtained in a compact form. Such explicit expression of sensitivities $\omega_{i}^{[n]}$, with $n=0, \ldots, 4$ and $i=1, \ldots, 6$ is reported in Appendices $\mathrm{B}$ and $\mathrm{C}$ for angular coordinate $\phi=0$.

\begin{tabular}{|c|c|c|c|c|c|c|c|c|c|c|}
\hline$r^{0}$ & $r^{1}$ & $r^{2}$ & $r^{3}$ & $r^{4}$ & $r^{5}$ & $r^{6}$ & $r^{7}$ & $r^{8}$ & $r^{9}$ & $r^{10}$ \\
\hline \multirow{5}{*}{$\omega_{1 \ldots 6}^{[0]}$} & \multirow{5}{*}{-} & \multirow{5}{*}{-} & \multirow{5}{*}{-} & \multirow{5}{*}{-} & \multirow{5}{*}{ - } & $\omega_{1,2}^{[1]}$ & - & $\begin{array}{l}\omega_{1}^{[2]} \\
\omega_{2}^{[2]}\end{array}$ & $\begin{array}{l}\omega_{1}^{[3]} \\
\omega_{2}^{[3]}\end{array}$ & $\begin{array}{c}\omega_{1}^{[4]} \\
\omega_{2}^{[4]}\end{array}$ \\
\hline & & & & & & $\omega_{3}^{[1]}$ & $\omega_{3}^{[2]}$ & $\omega_{3}^{[3]}$ & $\omega_{3}^{[4]}$ & $\cdots$ \\
\hline & & & & & & $\omega_{4}^{[1]}$ & $\omega_{4}^{[2]}$ & $\omega_{4}^{[3]}$ & $\omega_{4}^{[4]}$ & $\cdots$ \\
\hline & & & & & & $\omega_{5}^{[1]}$ & $\omega_{5}^{[2]}$ & $\omega_{5}^{[3]}$ & $\omega_{5}^{[4]}$ & $\ldots$ \\
\hline & & & & & & $\omega_{6}^{[1]}$ & $\omega_{6}^{[2]}$ & $\omega_{6}^{[3]}$ & $\omega_{6}^{[4]}$ & $\cdots$ \\
\hline
\end{tabular}

Table 1: Solution scheme to compute sensitivities $\omega_{i}^{[n]}$, with $n=1, \ldots, 4$ and $i=1, \ldots, 6$ from perturbation equation $G^{[n]}=0$ at the order $r^{n}$. Symbol "-" has the meaning of "indeterminate", while symbol "..." means "higher order unknowns". 


\section{Benchmark test: dispersion properties of SOFC-like devices}

One considers a multi-phase laminate, generated by the spatial repetition SOFC-like cell, whose periodic cell $\mathcal{A}$ is represented in figure 1 (b) and has dimensions $d_{1}=100 \mu \mathrm{m}$ and $d_{2}=440 \mu \mathrm{m}$. All phases are assumed to be linear isotropic and a plane problem characterized by conditions $\boldsymbol{\sigma} \mathbf{e}_{3}=\mathbf{0}, \mathbf{q} \cdot \mathbf{e}_{3}=0$, and $\mathbf{j} \cdot \mathbf{e}_{3}=0$ is considered, where $\mathbf{e}_{3}$ is a unit vector perpendicular to $\mathbf{e}_{1}$ and $\mathbf{e}_{2}$ to form a right handed base. Under these conditions the non vanishing components of micro constitutive tensors are

$$
\begin{aligned}
& C_{1111}^{m}=C_{2222}^{m}=\frac{E}{1-\nu^{2}}, \quad C_{1122}^{m}=\frac{\nu E}{1-\nu^{2}}, \quad C_{1212}^{m}=\frac{E}{2(1+\nu)}, \\
& K_{11}^{m}=K_{22}^{m}=K, \quad D_{11}^{m}=D_{22}^{m}=D, \\
& \alpha_{11}^{m}=\alpha_{22}^{m}=\alpha \frac{1-2 \nu}{1-\nu}, \quad \beta_{11}^{m}=\beta_{22}^{m}=\beta \frac{1-2 \nu}{1-\nu}
\end{aligned}
$$

where $E$ is the Young modulus, $\nu$ is the Poisson ratio, $K$ is the thermal conductivity constant, $D$ is the mass diffusivity constant, $\alpha$ is the thermal dilatation constant, and $\beta$ is the diffusive expansion constant. Ceramic electrolyte (phase 1) is considered made by yttria-stabilized zirconia (YSZ) having $E=155 G P a$,
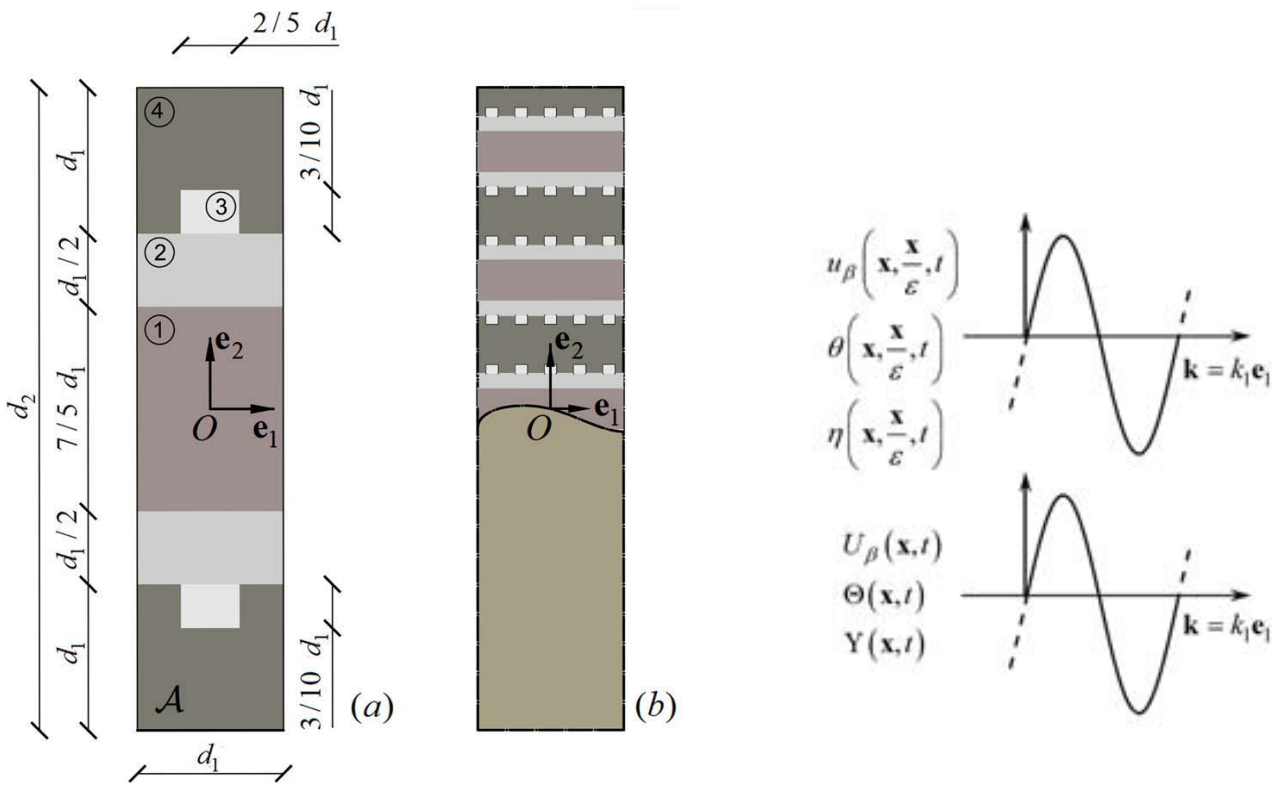

Figure 2: (a) draft of the SOFC-like periodic cell $\mathcal{A}$ having dimensions $d_{1}$ and $d_{2}$; (b) Waves propagation along direction $\mathbf{e}_{1}$ in the heterogeneous and in the equivalent first-order medium.

$\nu=0.3, K=0.0856 \mathrm{~W} /\left(\mathrm{m} K^{2}\right), D=0.614134 \mathrm{~kg} \mathrm{~s} / \mathrm{m}^{3}, \alpha=4.22375 \cdot 10^{3} \mathrm{~N} /\left(\mathrm{m}^{2} \mathrm{~K}\right), \beta=4.22375 \cdot 10^{2} \mathrm{~kg} / \mathrm{m}^{3}$ and inertial terms $\rho^{m}=5900 \mathrm{~kg} / \mathrm{m}^{3}, p^{m}=8412.8 \mathrm{~N} /\left(\mathrm{m}^{2} \mathrm{~K}^{2}\right)$ and $q^{m}=84128 \mathrm{~kg}^{2} /\left(\mathrm{m}^{3} \mathrm{~J}\right)$. Thermo-diffusive coupling constant $\psi^{m}$ is assumed to be equal to $280.427 \mathrm{~kg} /\left(\mathrm{m}^{3} \mathrm{~K}\right)$. Electrodes (phase 2) are considered made by nickel oxide (NiO) with $E=50 G P a, \nu=0.25, K=0.1570 \mathrm{~W} /\left(\mathrm{mK}^{2}\right), D=1.26072 \mathrm{~kg} \mathrm{~s} / \mathrm{m}^{3}, \alpha=1090$. $10^{3} \mathrm{~N} /\left(\mathrm{m}^{2} \mathrm{~K}\right), \beta=1090 \cdot 10^{2} \mathrm{~kg} / \mathrm{m}^{3}, \rho^{m}=6810 \mathrm{~kg} / \mathrm{m}^{3}, p^{m}=14008 \mathrm{~N} /\left(\mathrm{m}^{2} \mathrm{~K}^{2}\right), q^{m}=140080 \mathrm{~kg}^{2} /\left(\mathrm{m}^{3} \mathrm{~J}\right)$ and $\psi^{m}=1400.8 \mathrm{~kg} /\left(\mathrm{m}^{3} \mathrm{~K}\right)$. Finally, steel is supposed to constitute the conductive interconnections (phase 4) with $E=2.01 \cdot 10^{2} G P a, \nu=0.3, K=0.05 \mathrm{~W} /\left(\mathrm{mK}^{2}\right), D=6.8495 \cdot 10^{-6} \mathrm{~kg} \mathrm{~s} / \mathrm{m}^{3}, \alpha=5477 \cdot 10^{3} \mathrm{~N} /\left(\mathrm{m}^{2} \mathrm{~K}\right)$, $\beta=5477 \cdot 10^{2} \mathrm{~kg} / \mathrm{m}^{3}, \rho^{m}=8000 \mathrm{~kg} / \mathrm{m}^{3}, p^{m}=13699 \mathrm{~N} /\left(\mathrm{m}^{2} \mathrm{~K}^{2}\right), q^{m}=1369.9 \mathrm{~kg}^{2} /\left(\mathrm{m}^{3} \mathrm{~J}\right)$ and vanishing constant $\psi^{m}$. All constitutive properties of phase 3 representing the flow channels, are assumed to be equal to $1 / 10$ of the corresponding constitutive properties of electrodes. Perturbation functions $N_{k p q_{1}}^{(1)}, \tilde{N}_{k}^{(1)}, \hat{N}_{k}^{(1)}$,

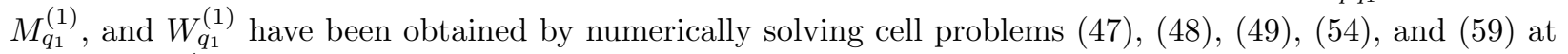
the order $\varepsilon^{-1}$. Numerical resolution has been obtained by means of a finite element procedure over the unit cell $\mathcal{Q}$, as detailed in Appendix E. Once perturbation functions are known, the overall constitutive tensors 
(68) are computed for the first-order thermo-diffusive homogenized medium and, exploiting the formalism described in Appendix D, they result

$$
\begin{aligned}
& \mathfrak{C}=\left[\begin{array}{ccc}
1.3927 & 0.3003 & 0 \\
0.3003 & 1.0595 & 0 \\
0 & 0 & 2 \cdot 0.6775
\end{array}\right] 10^{11} \frac{\mathrm{N}}{\mathrm{m}^{2}}, \quad \boldsymbol{K}=\left[\begin{array}{cc}
8.32 & 0 \\
0 & 6.45
\end{array}\right] 10^{-2} \frac{\mathrm{W}}{\mathrm{mK^{2 }}}, \\
& \boldsymbol{D}=\left[\begin{array}{cc}
5.392 & 0 \\
0 & 2.324
\end{array}\right] 10^{-1} \frac{\mathrm{kg} s}{\mathrm{~m}^{3}}, \quad \boldsymbol{\alpha}=\left[\begin{array}{c}
1.8454 \\
1.4822 \\
\sqrt{2} \cdot 0.0007
\end{array}\right] 10^{6} \frac{\mathrm{N}}{\mathrm{m}^{2} \mathrm{~K}}, \quad \boldsymbol{\beta}=\left[\begin{array}{c}
1.8454 \\
1.4822 \\
\sqrt{2} \cdot 0.0007
\end{array}\right] 10^{5} \frac{\mathrm{kg}}{\mathrm{m}^{3}}, \\
& \rho=6662.1 \frac{\mathrm{kg}}{\mathrm{m}^{3}}, \quad p=11428 \frac{\mathrm{N}}{\mathrm{m}^{2} \mathrm{~K}^{2}}, \quad q=114280 \frac{\mathrm{kg}}{\mathrm{m}^{3} \mathrm{~J}}, \quad \psi=4.1523 \cdot 10^{5} \frac{\mathrm{kg}}{\mathrm{m}^{3} \mathrm{~K}} .
\end{aligned}
$$

Generalized quadratic eigenvalue problem 90 has been solved in order to investigate the complex frequency spectrum of the periodic thermo-diffusive material varying the wave propagation direction $\mathbf{k}$. Defining the unit vector of propagation $\mathbf{m}=\mathbf{k} /\|\mathbf{k}\|_{2}$, two unit vectors of propagation are taken into account in the present example, namely $\mathbf{m}_{1}=\mathbf{e}_{1}$ parallel to the SOFC layering, and $\mathbf{m}_{2}=\mathbf{e}_{2}$ perpendicular to the first one. Dimensionless wave vector $\mathbf{k}^{*}=k_{1}^{*} \mathbf{e}_{1}+k_{2}^{*} \mathbf{e}_{2}$ is conveniently introduced, where dimensionless wave numbers $k_{1}^{*}=k_{1} d_{1}$ and $k_{2}^{*}=k_{2} d_{2}$ belong to the dimensionless first Brillouin zone $\mathcal{B}^{*}=[-\pi, \pi] \times[-\pi, \pi]$. MATLAB ${ }^{\circledR}$ has been used as a tool to solve the quadratic eigenvalue problem. It has been enhanced with the Advanpix Multiprecision Computing Toolbox which enables computing using an arbitrary precision. Matrices $\mathbf{H}_{2}$, $\mathbf{H}_{1}$ and $\mathbf{H}_{0}$ of problem 90 , in fact, result to be neither symmetric nor Hermitian and their entries are characterized by having absolute values differing by several orders of magnitudes. In this case the use of higher precision with respect to the standard double one, together with sparse representation of matrices, revealed to be crucial to get to the right final result. Figures 3 and 4 represent the complex spectrum obtained along directions $\mathbf{m}_{1}$ and $\mathbf{m}_{2}$, respectively. In particular, defining a reference frequency $\omega_{\text {ref }}=1 \mathrm{rad} / \mathrm{s}$ the dimensionless real part $\omega_{r}^{*}=\omega_{r} / \omega_{\text {ref }}$ and the dimensionless positive imaginary part $\omega_{i}^{*}=\omega_{i} / \omega_{\text {ref }}$ of the complex angular frequency, related to the attenuation and propagation mode, respectively, are represented in the two perpendicular directions as functions of the correspondent dimensionless wave number. Assuming $\boldsymbol{\alpha}=\mathbf{0}, \boldsymbol{\beta}=\mathbf{0}$ and $\psi=0$ in equations (89), blue curves of figures 3 and 4 are the dispersion curves of the homogenized first-order thermo-diffusive medium, computed as solutions of the quadratic generalized eigenvalue problem (90). This last gives rise to two pure damping modes, represented by the two parabolas in the plane $\omega_{i}^{*}=0$, and four pure propagation curves, complex conjugate in twos, plotted in the plane $\omega_{r}^{*}=0$. They are all acoustic branches departing from the origin of the reference system. Red curves in figures 3 and 4 describe the low frequencies branches of the complex frequency Floquet-Bloch spectrum relative to the heterogeneous thermo-diffusive SOFC-like material where all the four phases are characterized by vanishing coupling tensors $\boldsymbol{\alpha}^{m}$ and $\boldsymbol{\beta}^{m}$ and vanishing coupling constant $\psi^{m}$. Thanks to the periodicity of the medium, dispersion curves for the heterogeneous material have been obtained by solving the generalized quadratic eigenvalue problem 155 over the periodic cell $\mathcal{A}$, where this last is subjected to Floquet-Bloch, or quasiperiodicity, boundary conditions (Floquet, 1883, Bloch, 1929, Brillouin, 1953; Mead, 1973, Langley, 1993) . The procedure adopted to obtain the complex frequency band structure for the heterogeneous material is outlined in detail in Appendix E. Figures 3 (b) and 4 (b) are a zoom of the correspondent three dimensional spectra 3 (a) and 4 (a) considering $0 \leq k_{i}^{*} \leq \pi / 3(i=1,2)$. Plane $\omega_{i}^{*}=0$ is represented in figure 3.(c) along direction $\mathbf{m}_{1}$ and in figure 4 (c) along $\mathbf{m}_{2}$. Analogously, planes $\omega_{r}^{*}=0$ are plotted in figures 3 (d) and 4 (d). As one can notice, a very good agreement between the first branches of the spectrum of the heterogeneous material and the ones of homogenized medium, is achieved for $0 \leq k_{i}^{*} \leq \pi / 3 \quad(i=1,2)$. A decrease of the accuracy is generally expected for $k_{i}^{*} \geq \pi / 3$ as a first-order approximation is adopted to describe the equivalent thermo-diffusive medium behavior and obtained results confirm this fact. Furthermore, the obtained approximation of the complex frequency band structure results to be more accurate along the $\mathbf{m}_{2}$ direction than along $\mathbf{m}_{1}$ and superior performances attained in the direction perpendicular to the material layering is confirmed by previous results achieved in the literature (Bacigalupo and Gambarotta, 2014). No partial gaps are detected along $\mathbf{m}_{1}$ and $\mathbf{m}_{2}$ in the frequency ranges taken into account. Figure 5 represents dispersion curves obtained in the plane $\omega_{i}^{*}=0$ along directions $\mathbf{m}_{1}$ (figure 5.(a)) and $\mathbf{m}_{2}$ (figure 5f(b)) when thermo-diffusive coupling constant $\psi^{m}$ is introduced such that $\psi^{m}=2804.27 \mathrm{~kg} /\left(\mathrm{m}^{3} \mathrm{~K}\right)$ for phase $1, \psi^{m}=14008 \mathrm{~kg} /\left(\mathrm{m}^{3} \mathrm{~K}\right)$ for phases 2 and $\psi^{m}=1400.8 \mathrm{~kg} /\left(\mathrm{m}^{3} \mathrm{~K}\right)$ for phase 3 . Figure 5 confirms the capabilities of the proposed first-order asymptotic procedure to approximate dispertion properties of thermodiffusive materials in the low frequency regime. A comparison with the two relative spectra in the case of 

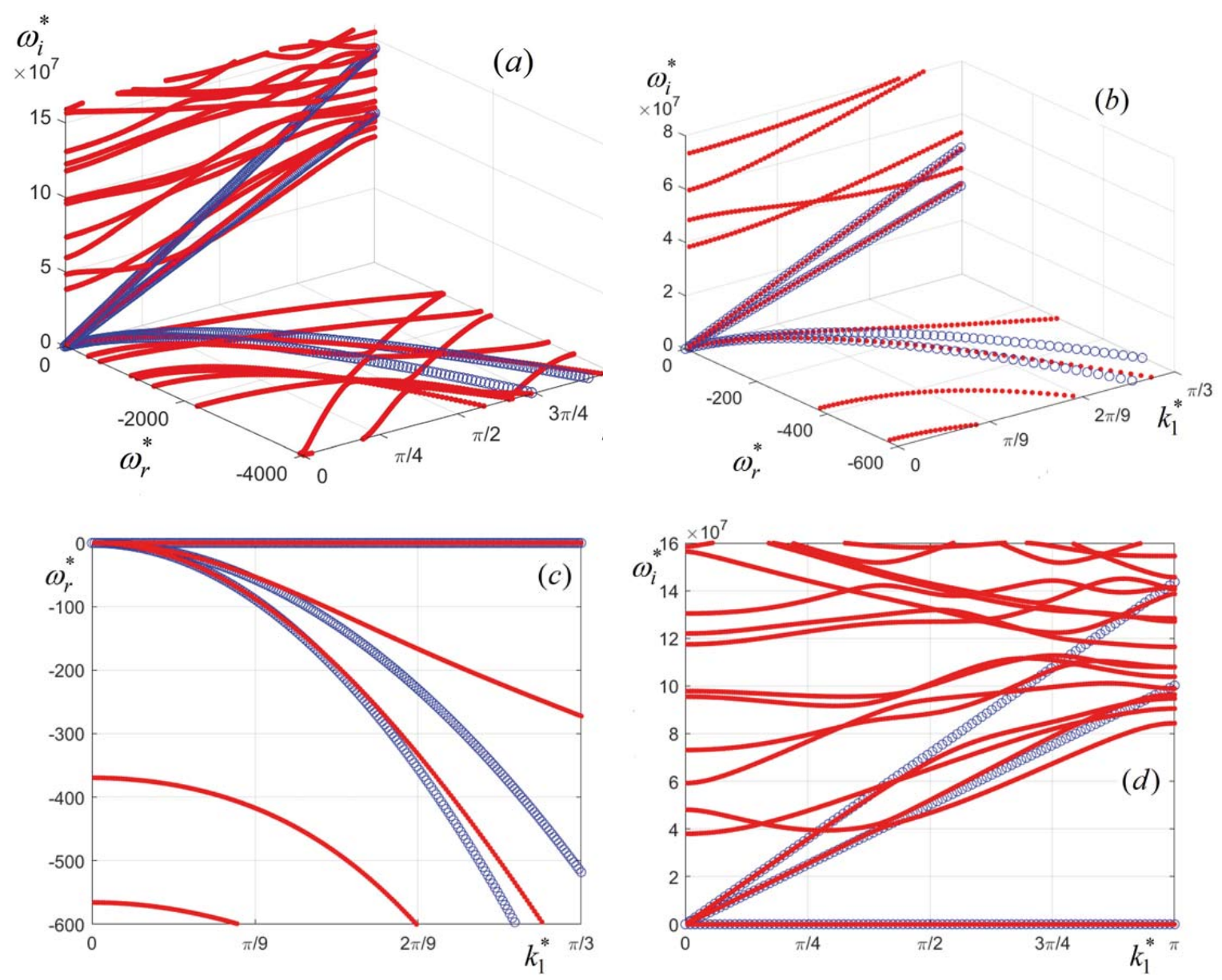

Figure 3: Complex frequency spectrum of the heterogeneous SOFC-like material (red curves) and of the firstorder equivalent medium (blue curves) along direction $\mathbf{m}_{1}$ for vanishing coupling coefficients. (a) $\omega_{r}^{*}$ and $\omega_{i}^{*}$ vs $k_{1}^{*}$; (b) zoomed view of the $3 D$ spectrum considering $0 \leq k_{1}^{*} \leq \pi / 3$; (c)damping modes in the plane $\omega_{r}^{*}-k_{1}^{*}$; (d) propagation modes in the plane $\omega_{i}^{*}-k_{1}^{*}$.

vanishing $\psi^{m}$ (figures 3 (c) and 4 (c)) brings to light the qualitative differences spotted in the two cases between the spectra relative to the heterogeneous material. In particular, veering phenomena, meaning the repulsion between two branches, are accentuated in the case of non vanishing $\psi^{m}$, and, in the correspondence of the same $k_{i}^{*}$, the absolute values of $\omega_{r}^{*}$ increases for each branch of the spectrum.

When all overall coupling tensors $\boldsymbol{\alpha}$ and $\boldsymbol{\beta}$ and overall coupling constant $\psi$ are taken into account with their value as expressed in equation (97), dispersion curves for the homogenized first-order medium have the behavior illustrated in figure 6f(a) along $\mathbf{m}_{1}$ and in figure 6f(c) along $\mathbf{m}_{2}$ (blue curves). When coupling coefficients are taken into consideration, resolution of quadratic generalized eigenvalue problem (90) provides two pure damping branches and four (complex conjugate in twos) mixed mode branches having both components $\omega_{i}^{*}$ and $\omega_{r}^{*}$ different from zero. Red dots in figure 6 represent dispersion properties of the equivalent medium obtained by means of the asymptotic approximation procedure described in Section 6.1. which allows to achieve a compact and explicit parametric approximation of the eigenvalues in terms of the constitutive coefficients of the homogenized continuum. In particular, a fourth order approximation of type (95) is achieved along both $\mathbf{m}_{1}$ and $\mathbf{m}_{2}$ by solving recursive perturbation problems $G^{[n]}(r=0)=0$ at the order $k_{i}^{n}(i=1,2)$ in accordance with the solution scheme described in table 1. Figures 6f(b) and 6.(d) represent, respectively, the complex spectrum obtained along the two perpendicular directions $\mathbf{m}_{1}$ and $\mathbf{m}_{2}$ when components of the coupling tensors $\boldsymbol{\alpha}$ and $\boldsymbol{\beta}$ and the value of $\psi$ are multiplied by $10^{2}$. When the absolute values of coupling tensors increases, mixed mode branches bend toward the plane $\omega_{i}^{*}=0$ increasing their damping component, and pure attenuation modes bend toward the axis $\omega_{i}^{*}=0$, yet remaining in the 

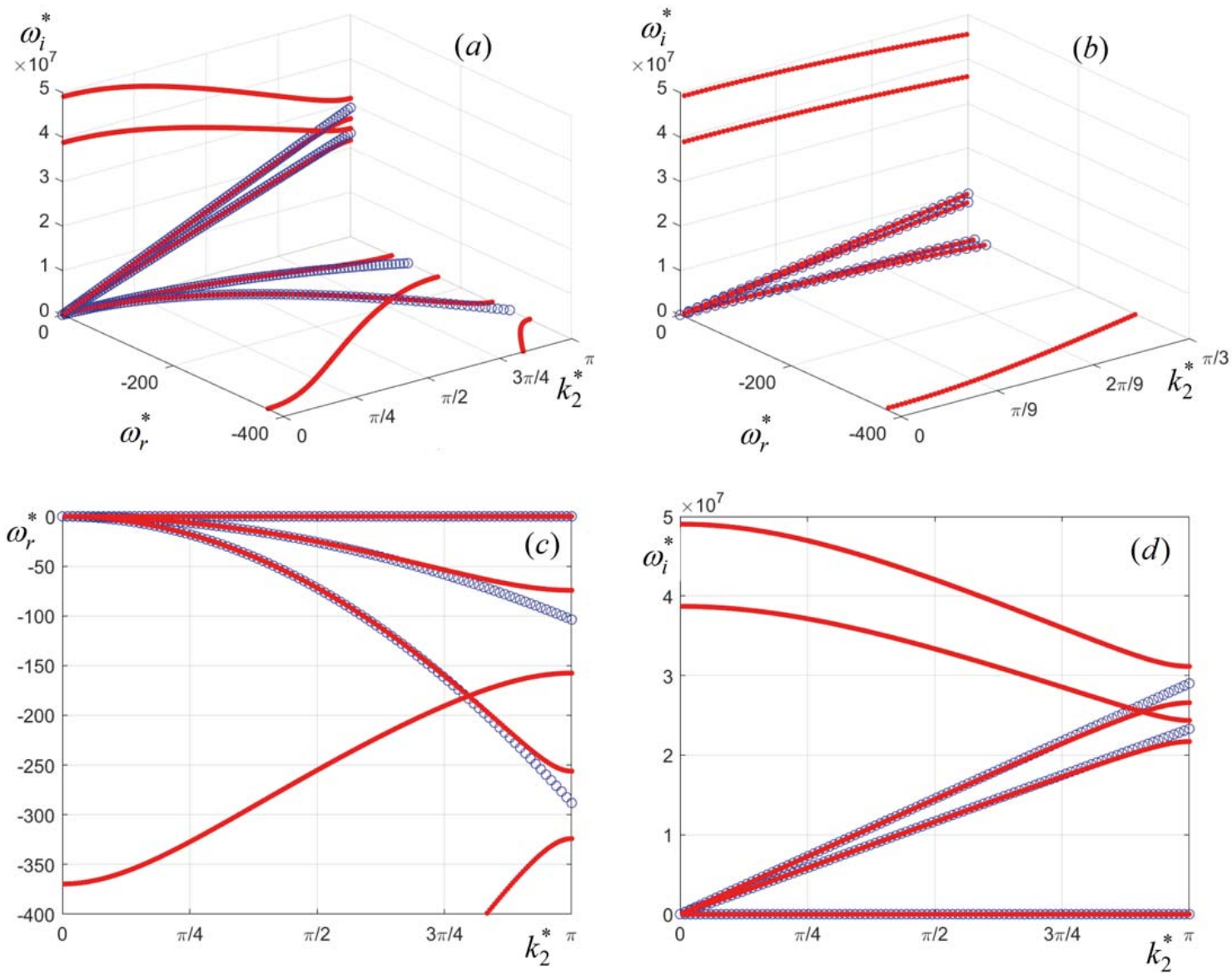

Figure 4: Complex frequency spectrum of the heterogeneous SOFC-like material (red curves) and of the firstorder equivalent medium (blue curves) along direction $\mathbf{m}_{2}$ for vanishing coupling coefficients. (a) $\omega_{r}^{*}$ and $\omega_{i}^{*}$ vs $k_{2}^{*}$; (b) zoomed view of the $3 D$ spectrum considering $0 \leq k_{2}^{*} \leq \pi / 3$; (c)damping modes in the plane $\omega_{r}^{*}-k_{2}^{*} ;$ (d) propagation modes in the plane $\omega_{i}^{*}-k_{2}^{*}$.
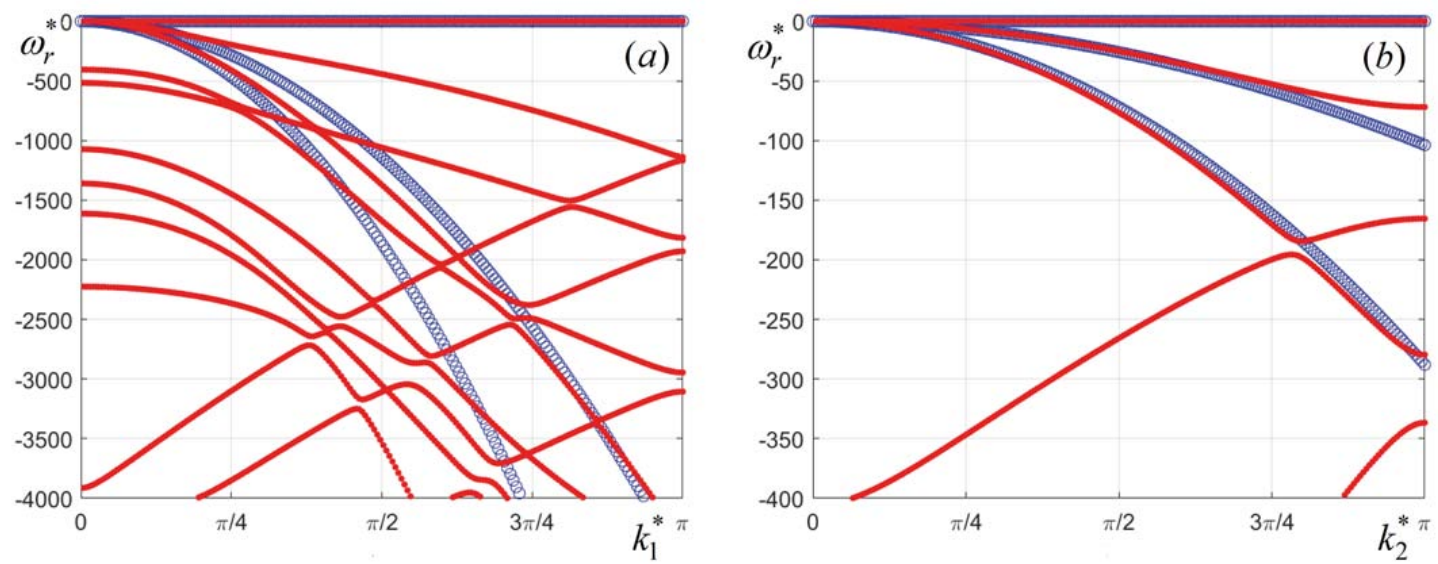

Figure 5: Real part of complex frequency for the heterogeneous SOFC-like material (red curves) and for the first-order equivalent medium (blue curves) in the case of non vanishing thermo-diffusive coupling coefficient $\psi$. (a) Propagation along direction $m_{1}$; (b) propagation along direction $\mathbf{m}_{2}$. 
plane $\omega_{r}^{*}=0$. As one can notice, the perfect agreement obtained between the eigenvalues of problem 90 and their asymptotic approximation (see figures 6 (a) and 6 f(c)) deteriorates as the coupling increases as shown in figures 6-(b) and 6f(d), preserving nevertheless the accuracy of the approximation for $0 \leq k_{i}^{*} \leq \pi / 3(i=1,2)$, as expected.
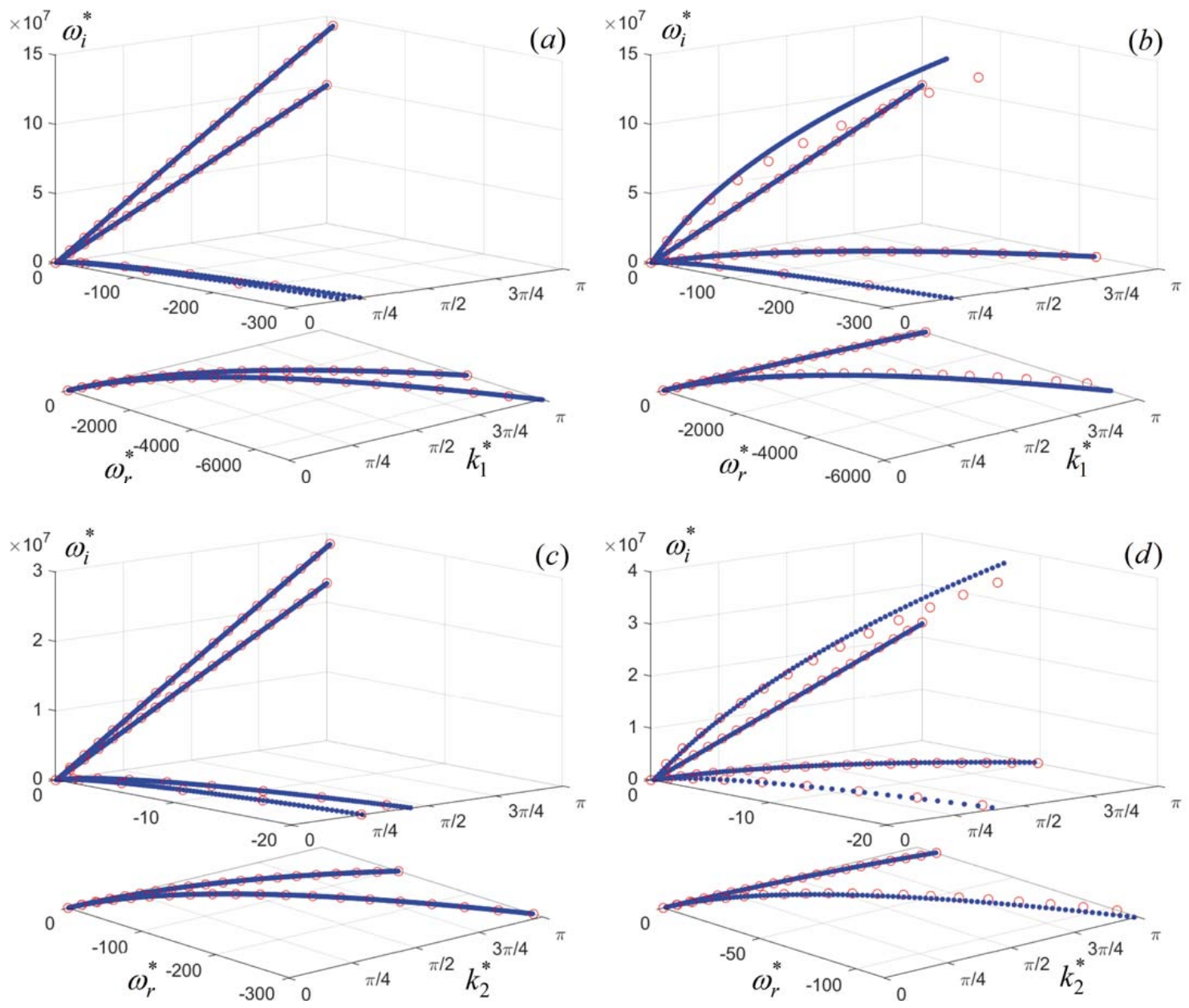

Figure 6: Complex frequency spectrum of the first-order equivalent medium (blue curves) and its fourthorder perturbative approximation (red dots) as described in equation (95). Each subfigure is enhanced with a perspective view of the plane $\omega_{i}^{*}=0$ showing the complete range of values for $\omega_{r}^{*}$. Propagation along direction $\mathbf{m}_{1}$ and $\boldsymbol{\alpha}, \boldsymbol{\beta}$ and $\psi$ with components as expressed in $(97)$ (a) and increased by a factor $10^{2}$ (b). Propagation along direction $\mathbf{m}_{2}$ and $\boldsymbol{\alpha}, \boldsymbol{\beta}$ and $\psi$ with components as expressed in (97) (c) and increased by a factor $10^{2}$ (d).

\section{Conclusions}

The present work is devoted to the formulation of an asymptotic homogenization technique for periodic microstructured materials characterized by thermo-diffusive phenomena. The aim of the proposed technique is twofold: it allows determining the overall constitutive properties of the first-order equivalent medium and to investigate its complex frequency spectrum by providing its dispersion curves. Down-scaling relations are determined, which relate the three microfields, namely displacement, relative temperature and chemical potential to the corresponding macrostructural ones and to their gradients by means of perturbation functions. These lasts are regular, $\mathcal{Q}$-periodic functions, which take into account the effects of microstructural heterogeneities. They are solutions of recursive, non homogeneous differential problems, known as cell problems, obtained inserting asymptotic expansions of the microfields in powers of the microstructural characteristic 
size $\varepsilon$ into micro governing field equations and reordering at the different orders of $\varepsilon$. Substitution of downscaling relations into local balance equations provides the average field equations of infinite order, whose formal solution can be obtained by inserting an asymptotic expansion of the macrofields in powers of $\varepsilon$ and reordering at the different orders of $\varepsilon$. The attained zeroth order differential problems yield to the governing field equations for the equivalent first-order (Cauchy) thermo-diffusive medium whose overall constitutive tensors are provided in closed form.

By means of proper integral transforms of such global balance equations a quadratic generalized eigenvalue problem derives, whose solution provides the complex frequency spectrum of the first-order homogeneous material in the first Brillouin zone. In order to assess the capabilities of the presented dynamic asymptotic homogenization technique, a generalization of the Floquet-Bloch theory has been implemented in order to investigate dispersion properties of the heterogeneous thermo-diffusive medium. Thanks to the periodicity of the microstructured material, a quadratic generalized eigenvalue problem is solved over the periodic cell subjected to Floquet-Bloch boundary conditions. The eigenvalues provide the imaginary and real components of the angular frequency, related respectively to the propagation and attenuation modes of the wave that propagates inside the medium, as functions of the wave vector. The very good matching obtained between dispersion curves of the first-order homogenized continuum and the lowest frequency ones relative to the heterogeneous medium, confirms the accuracy of the proposed homogenization technique in predicting the behavior of the acoustic branches of the complex spectrum of the material under consideration, at least in the range of wave number values admissible for a first-order approximation.

Furthermore, an asymptotic approximation of the complex spectrum is here presented based upon the resolution of recursive perturbation problems at the different orders of $r$, here intended as the Euclidean norm of the wave vector. Perturbation problems derive from a Taylor series expansion of the implicit characteristic equation of the equivalent medium in the transformed space and frequency domain and their solutions provide the sensitivities of the eigenvalues at the different orders of $r$. Parametric approximation of the complex angular frequency allows obtaining a compact analytical solution of the characteristic equation, in which the dependence upon the overall constitutive coefficients is made explicit. A fourth order asymptotic approximation of the spectrum demonstrates to be in good agreement with dispersion curves of the homogenized material, also in the case of increased coupling coefficients of field equations.

In the context of renewable energy devises, numerical experiments have been conducted referring to a Solid Oxide Fuel Cell (SOFC)-like material, whose typical building block can be modeled as a periodic thermo-diffusive elastic multi-layered material. SOFC are typically subjected to high operating temperatures and to intensive ions flows, which can increase their vulnerability to damage and undermine their efficiency. A correct prediction of their behavior is therefore of fundamental importance in order to design high performances batteries. When scale separation holds, homogenization techniques reveal to be particularly useful to obtain an accurate, but concise at the same time, description of the material, both in static and dynamic regime. In this regard, proposed multifield asymptotic homogenization is an efficient and rigorous tool for the investigation of thermo-diffusive materials having periodic microstructure. When non local phenomena connected to the microstructural length scale and/or size effects come into play, first-order homogenization methods result to be inadequate in approximating the behavior of the periodic material. In these cases more accurate approximations could be obtained by considering higher-order cell problems. Alternatively, homogenized higher-order materials can be properly modeled by means of non local higher-order homogenization approaches, which allow to consider a characteristic length scale linked to microstructural effects, but the employment of such techniques is out of the scope of the present study.

\section{References}

Addessi, D., De Bellis, M., Sacco, E., 2013. Micromechanical analysis of heterogeneous materials subjected to overall cosserat strains. Mechanics Research Communications 54, 27-34.

Allaire, G., 1992. Homogenization and two-scale convergence. SIAM Journal of Mathematical Analisys 23, $1482-1518$.

Atkinson, A., Sun, B., 2007. Residual stress and thermal cycling of planar solid oxide fuel cells. Materials Science and Technology 23, 1135-1143. 
Bacca, M., Bigoni, D., Dal Corso, F., Veber, D., 2013a. Mindlin second-gradient elastic properties from dilute two-phase cauchy-elastic composites. part i: Closed form expression for the effective higher-order constitutive tensor. International Journal of Solids and Structures 50(24), 4010-4019.

Bacca, M., Bigoni, D., Dal Corso, F., Veber, D., 2013b. Mindlin second-gradient elastic properties from dilute two-phase cauchy-elastic composites part ii: Higher-order constitutive properties and application cases. international journal of solids and structures. International Journal of Solids and Structures 50(24), 4020-4029.

Bacca, M., Dal Corso, F., Veber, D., Bigoni, D., 2013c. Anisotropic effective higher-order response of heterogeneous cauchy elastic materials. Mechanics Research Communications 54, 63-71.

Bacigalupo, A., 2014. Second-order homogenization of periodic materials based on asymptotic approximation of the strain energy: formulation and validity limits. Meccanica 49(6), 1407-1425.

Bacigalupo, A., De Bellis, M.L., Gnecco, G., 2019. Complex frequency band structure of periodic thermodiffusive materials by floquet-bloch theory. Acta Mechanica 230, 3339-3363.

Bacigalupo, A., Gambarotta, L., 2010. Second-order computational homogenization of heterogeneous materials with periodic microstructure. ZAMM-Journal of Applied Mathematics and Mechanics/Zeitschrift fr Angewandte Mathematik und Mechanik 90, 796-811.

Bacigalupo, A., Gambarotta, L., 2014. Computational dynamic homogenization for the analysis of dispersive waves in layered rock masses with periodic fractures. Computers and Geotechnics 56, 61-68.

Bacigalupo, A., Lepidi, M., 2016. High-frequency parametric approximation of the floquet-bloch spectrum for anti-tetrachiral materials. International Journal of Solids and Structures 97, 575-592.

Bacigalupo, A., Morini, L., Piccolroaz, A., 2014. Effective elastic properties of planar sofcs: A non-local dynamic homogenization approach. International Journal of Hydrogen Energy 39(27), 15017-15030.

Bacigalupo, A., Morini, L., Piccolroaz, A., 2016. Multiscale asymptotic homogenization analysis of thermodiffusive composite materials. International Journal of Solids and Structures 85-86, 15-33.

Bacigalupo, A., Paggi, M., Dal Corso, F., Bigoni, D., 2018. Identification of higher-order continua equivalent to a cauchy elastic composite. Mechanics Research Communications 93, 11-22.

Bakhvalov, N., Panasenko, G., 1984. Homogenization: Averaging Processes in Periodic Media. Kluwer Academic Publishers, Dordrecht-Boston-London.

Bensoussan, A., Lions, J., Papanicolaou, G., 1978. Asymptotic analysis for periodic structures. NorthHolland, Amsterdam.

Bigoni, D., Drugan, W., 2007. Analytical derivation of cosserat moduli via homogenization of heterogeneous elastic materials. Journal of Applied Mechanics 74(4), 741-753.

Bloch, F., 1929. Über die quantenmechanik der elektronen in kristallgittern. Zeitschrift für physik 52, $555-600$.

Bove, R., Ubertini, S., 2008. Modeling solid oxide fuel cells: methods, procedures and techniques. Springer Science \& Business Media.

Brandon, N., Brett, D., 2006. Engineering porous materials for fuel cell applications. Philosophical Transactions of the Royal Society A: Mathematical, Physical and Engineering Sciences 364, 147-159.

Brillouin, L., 1953. Wave propagation in periodic structures: electric filters and crystal lattices .

Colpan, C.O., Dincer, I., Hamdullahpur, F., 2008. A review on macro-level modeling of planar solid oxide fuel cells. International Journal of Energy Research 32, 336-355.

De Bellis, M.L., Addessi, D., 2011. A cosserat based multi-scale model for masonry structures. International Journal for Multiscale Computational Engineering 9, 543. 
Delette, G., Laurencin, J., Usseglio-Viretta, F., Villanova, J., Bleuet, P., Lay-Grindler, E., Le Bihan, T., 2013. Thermo-elastic properties of sofc/soec electrode materials determined from three-dimensional microstructural reconstructions. International journal of hydrogen energy 38, 12379-12391.

Dev, B., Walter, M.E., Arkenberg, G.B., Swartz, S.L., 2014. Mechanical and thermal characterization of a ceramic/glass composite seal for solid oxide fuel cells. Journal of Power Sources 245, 958-966.

Fantoni, F., Bacigalupo, A., Paggi, M., 2017. Multi-field asymptotic homogenization of thermo-piezoelectric materials with periodic microstructure. International Journal of Solids and Structures 120, 31-56.

Fantoni, F., Bacigalupo, A., Paggi, M., 2018. Design of thermo-piezoelectric microstructured bending actuators via multi-field asymptotic homogenization. International Journal of Mechanical Sciences 146, $319-336$.

Feyel, F., 2003. A multilevel finite element method (fe2) to describe the response of highly non-linear structures using generalized continua. Computer Methods in applied Mechanics and engineering 192, $3233-3244$.

Feyel, F., Chaboche, J., 2000. FE ${ }^{2}$ multiscale approach for modelling the elastoviscoplastic behaviour of long fibre $\mathrm{SiC} / \mathrm{Ti}$ composite materials. Computer Methods in Applied Mechanics Engineering 183, 309-330.

Floquet, G., 1883. Sur les équations différentielles linéaires à coefficients périodiques, in: Annales scientifiques de l'École normale supérieure, pp. 47-88.

Forest, S., 2002. Homogenization methods and the mechanics of generalized continua-part 2. Theoretical and applied mechanics 28, 113-144.

Forest, S., Sab, K., 1998. Cosserat overall modeling of heterogeneous materials. Mechanics Research Communications 25(4), 449-454.

Forest, S., Trinh, D., 2011. Generalized continua and nonhomogeneous boundary conditions in homogenisation methods. ZAMMJournal of Applied Mathematics and Mechanics/Zeitschrift fr Angewandte Mathematik und Mechanik 91(2), 90-109.

Gambin, B., Kröner, E., 1989. Higher order terms in the homogenized stressstrain relation of periodic elastic media. physica status solidi (b). International Journal of Engineering Science 151(2), 513-519.

Hajimolana, S.A., Hussain, M.A., Daud, W.A.W., Soroush, M., Shamiri, A., 2011. Mathematical modeling of solid oxide fuel cells: A review. Renewable and Sustainable Energy Reviews 15, 1893-1917.

Hasanov, R., Smirnova, A., Gulgazli, A., Kazimov, M., Volkov, A., Quliyeva, V., Vasylyev, O., Sadykov, V., 2011. Modeling design and analysis of multi-layer solid oxide fuel cells. International journal of hydrogen energy $36,1671-1682$.

Kaczmarczyk, L., Pearce, C.J., Bićanić, N., 2008. Scale transition and enforcement of rve boundary conditions in second-order computational homogenization. International Journal for Numerical Methods in Engineering 74, 506-522.

Kakac, S., Pramuanjaroenkij, A., Zhou, X.Y., 2007. A review of numerical modeling of solid oxide fuel cells. International journal of hydrogen energy $32,761-786$.

Kim, J.H., Liu, W.K., Lee, C., 2009. Multi-scale solid oxide fuel cell materials modeling. Computational Mechanics 44, 683-703.

Kouznetsova, V., Geers, M., Brekelmans, W., 2002. Multi-scale constitutive modelling of heterogeneous materials with a gradient-enhanced computational homogenization scheme. International Journal for Numerical Methods in Engineering 54, 1235-1260.

Kouznetsova, V., Geers, M., Brekelmans, W., 2004. Multi-scale second-order computational homogenization of multi-phase materials: a nested finite element solution strategy. Computer Methods in Applied Mechanics and Engineering 193(48), 5525-5550. 
Kuebler, J., Vogt, U.F., Haberstock, D., Sfeir, J., Mai, A., Hocker, T., Roos, M., Harnisch, U., 2010. Simulation and validation of thermo-mechanical stresses in planar sofcs. Fuel Cells 10, 1066-1073.

Langley, R., 1993. A note on the force boundary conditions for two-dimensional periodic structures with corner freedoms. Journal of Sound and Vibration 167, 377-381.

Lew, T., Scarpa, F., Worden, K., 2004. Homogenisation metamodelling of perforated plates. Strain 40, $103-112$.

Mead, D., 1973. A general theory of harmonic wave propagation in linear periodic systems with multiple coupling. Journal of Sound and Vibration 27, 235-260.

Mehrabadi, M., Cowin, S., 1990. Eigentensors of linear anisotropic elastic materials. The Quarterly Journal of Mechanics and Applied Mathematics 43(1), 15-41.

Milton, G.W., Willis, J.R., 2007. On modifications of newton's second law and linear continuum elastodynamics. Proceedings of the Royal Society A: Mathematical, Physical and Engineering Sciences 463, $855-880$.

Molla, T.T., Kwok, K., Frandsen, H.L., 2016. Efficient modeling of metallic interconnects for thermomechanical simulation of sofc stacks: homogenized behaviors and effect of contact. International Journal of Hydrogen Energy 41, 6433-6444.

Muramatsu, M., Terada, K., Kawada, T., Yashiro, K., Takahashi, K., Takase, S., 2015. Characterization of time-varying macroscopic electro-chemo-mechanical behavior of sofc subjected to ni-sintering in cermet microstructures. Computational Mechanics 56, 653-676.

Nassar, H., He, Q.C., Auffray, N., 2015. Willis elastodynamic homogenization theory revisited for periodic media. Journal of the Mechanics and Physics of Solids 77, 158-178.

Nowacki, W., 1974a. Dynamical problem of thermodiffusion in solids. 1. Bulletin de lácademie polonaise des sciences-serie des sciences techniques $22,55-64$.

Nowacki, W., 1974b. Dynamical problem of thermodiffusion in solids. 2. Bulletin de lácademie polonaise des sciences-serie des sciences techniques 22, 205-211.

Nowacki, W., 1974c. Dynamical problem of thermodiffusion in solids. 3. Bulletin de lácademie polonaise des sciences-serie des sciences techniques 22, 257-266.

Ostoja-Starzewski, M., Boccara, S.D., Jasiuk, I., 1999. Couple-stress moduli and characteristic length of a two-phase composite. Mechanics Research Communications 26, 387-396.

Paley, R., Wiener, N., 1934. Fourier transforms in the complex domain. volume 19. American Mathematical Soc.

Peerlings, R., Fleck, N., 2004. Computational evaluation of strain gradient elasticity constants. International Journal for Multiscale Computational Engineering 2(4).

Phani, A.S., Woodhouse, J., Fleck, N., 2006. Wave propagation in two-dimensional periodic lattices. The Journal of the Acoustical Society of America 119, 1995-2005.

Pitakthapanaphong, S., Busso, E., 2005. Finite element analysis of the fracture behaviour of multi-layered systems used in solid oxide fuel cell applications. Modelling and Simulation in Materials Science and Engineering 13, 531 .

Salvadori, A., Bosco, E., Grazioli, D., 2014. A computational homogenization approach for Li-ion battery cells. Part 1 - Formulation. Journal of the Mechanics and Physics of Solids 65, 114-137. doi http: //dx.doi.org/10.1016/j.jmps.2013.08.010.

Sanchez-Palencia, E., 1974. Comportements local et macroscopique d'un type de milieux physiques heterogenes. International Journal of Engineering Science 12(4), 331-351. 
Scarpa, F., Adhikari, S., Phani, A.S., 2009. Effective elastic mechanical properties of single layer graphene sheets. Nanotechnology 20, 065709.

Smyshlyaev, V., Cherednichenko, K., 2000. On rigorous derivation of strain gradient effects in the overall behaviour of periodic heterogeneous media. Journal of the Mechanics and Physics of Solids 48(6), 13251357.

Trovalusci, P., Ostoja-Starzewski, M., De Bellis, M.L., Murrali, A., 2015. Scale-dependent homogenization of random composites as micropolar continua. European Journal of Mechanics-A/Solids 49, 396-407.

Yuan, X., Tomita, Y., Andou, T., 2008. A micromechanical approach of nonlocal modeling for media with periodic microstructures. Mechanics Research Communications 35, 126-133.

Zäh, D., Miehe, C., 2013. Computational homogenization in dissipative electro-mechanics of functional materials. Computer Methods in Applied Mechanics and Engineering 267, 487-510.

Zhu, W., Deevi, S., 2003. A review on the status of anode materials for solid oxide fuel cells. Materials Science and Engineering: A 362, 228-239.

\section{Appendix A. Proof of equivalence among thermo-diffusive homo- geneous tensors}

In the present Section the following equivalences between the components of overall constitutive tensors that appear in the average field equations of infinite order (67) are demonstrated in detail

$$
\tilde{n}_{p q_{1}}^{(2)}=\tilde{m}_{p q_{1}}^{(2,1)}, \quad \hat{n}_{p q_{1}}^{(2)}=\tilde{w}_{p q_{1}}^{(2,1)}, \quad \hat{m}^{(2,1)}=\hat{w}^{(2,1)} .
$$

This allows relating components of tensors $\tilde{\mathbf{n}}^{(2)}=\tilde{n}_{p q_{1}}^{(2)} \mathbf{e}_{p} \otimes \mathbf{e}_{q_{1}}, \tilde{\mathbf{m}}^{(2,1)}=\tilde{m}_{p q_{1}}^{(2,1)} \mathbf{e}_{p} \otimes \mathbf{e}_{q_{1}}, \hat{\mathbf{n}}^{(2)}=\hat{n}_{p q_{1}}^{(2)} \mathbf{e}_{p} \otimes \mathbf{e}_{q_{1}}$, $\tilde{\mathbf{w}}^{(2,1)}=\tilde{w}_{p q_{1}}^{(2,1)} \mathbf{e}_{p} \otimes \mathbf{e}_{q_{1}}$ to the corresponding ones of overall constitutive tensors $\boldsymbol{\alpha}$ and $\boldsymbol{\beta}$ and to relate constants $\hat{m}^{(2,1)}$ and $\hat{w}^{(2,1)}$ to the overall coupling constant $\psi$.

Proof of $\tilde{\mathbf{n}}^{(2)}=\tilde{\mathbf{m}}^{(2,1)}$

Components of tensors $\tilde{\mathbf{n}}^{(2)}$ and $\tilde{\mathbf{m}}^{(2,1)}$ come from the known terms of cell problems (51) and (56), respectively, and have the following expressions

$$
\begin{aligned}
& \tilde{n}_{p q_{1}}^{(2)}=\left\langle\alpha_{p q_{1}}^{m}-C_{p q_{1} k j, j}^{m} \tilde{N}_{k, j}^{(1)}\right\rangle, \\
& \tilde{m}_{p q_{1}}^{(2,1)}=\left\langle\alpha_{p q_{1}}^{m}+\alpha_{i q_{2}}^{m} N_{i p q_{1}, q_{2}}^{(1)}\right\rangle .
\end{aligned}
$$

The weak form of the first mechanical cell problem 47 at the order $\varepsilon^{-1}$

$$
\left(C_{p j k l}^{m} N_{k i q_{1}, l}^{(1)}\right)_{, j}+C_{p j i q_{1}, j}^{m}=0
$$

can be written in the following way, considering as test function the perturbation function $\tilde{N}_{p}^{(2)}$

$$
\left\langle\left[\left(C_{p j k l}^{m} N_{k i q_{1}, l}^{(1)}\right)_{, j}+C_{p j i q_{1}, j}^{m}\right] \tilde{N}_{p}^{(1)}\right\rangle=0 .
$$

Divergence theorem and $\mathcal{Q}$-periodicity of micro constitutive tensors and perturbation functions, allow writing equation (101) as

$$
\left\langle\left[C_{p j k l}^{m} N_{k i q_{1}, l}^{(1)}+C_{p j i q_{1}}^{m}\right] \tilde{N}_{p, j}^{(1)}\right\rangle=0 .
$$

Adding vanishing term (102) to expression 99a), one obtains

$$
\tilde{n}_{p q_{1}}^{(2)}=\left\langle\alpha_{p q_{1}}^{m}-C_{p q_{1} i l, l}^{m} \tilde{N}_{i, l}^{(1)}+C_{i j k l}^{m} N_{i p q_{1}, j}^{(1)} \tilde{N}_{k, l}^{(1)}+C_{k l p q_{1}}^{m} \tilde{N}_{k, l}^{(1)}\right\rangle=\left\langle\alpha_{p q_{1}}^{m}+C_{k l i k}^{m} \tilde{N}_{k, l}^{(1)} N_{i p q_{1}, j}^{(1)}\right\rangle .
$$


Analogously, from the second mechanical cell problem 48 at the order $\varepsilon^{-1}$

$$
\left(C_{i j k l}^{m} \tilde{N}_{k, l}^{(1)}\right)_{, j}-\alpha_{i j, j}^{m}=0
$$

the following weak form can be written in terms of test function $N_{i p q_{1}}^{(1)}$

$$
\left\langle\left[\left(C_{i j k l}^{m} \tilde{N}_{k, l}^{(1)}\right)_{, j}-\alpha_{i j, j}^{m}\right] N_{i p q_{1}}^{(1)}\right\rangle=0 .
$$

Expression 105 can be transformed into

$$
\left\langle\left[C_{i j k l}^{m} \tilde{N}_{k, l}^{(1)}-\alpha_{i j}^{m}\right] N_{i p q_{1}, j}^{(1)}=0\right\rangle,
$$

thanks to divergence theorem and $\mathcal{Q}$-periodicity of micro tensors and perturbation functions. Adding 106 to $99 \mathrm{~b}$ yields

$$
\tilde{m}_{p q_{1}}^{(2,1)}=\left\langle\alpha_{p q_{1}}^{m}+\alpha_{i q_{2}}^{m} N_{i p q_{1}, q_{2}}^{(1)}+C_{i j k l}^{m} \tilde{N}_{k, l}^{(1)} N_{i p q_{1}, j}^{(1)}-\alpha_{i j}^{m} N_{i p q_{1}, j}^{(1)}\right\rangle=\left\langle\alpha_{p q_{1}}^{m}+C_{i j k l}^{m} \tilde{N}_{k, l}^{(1)} N_{i p q_{1}, j}^{(1)}\right\rangle,
$$

from which identity $\hat{n}_{p q_{1}}^{(2)}=\tilde{w}_{p q_{1}}^{(2,1)}$ follows.

Proof of $\hat{\mathbf{n}}^{(2)}=\tilde{\mathbf{w}}^{(2,1)}$

Components $n_{p q_{1}}^{(2)}$ and $\tilde{w}_{p q_{1}}^{(2)}$ are related to the known terms of cell problems 52 and 62 , namely

$$
\begin{aligned}
& \hat{n}_{p q_{1}}^{(2)}=\left\langle\beta_{p q_{1}}^{m}-C_{p q_{1} k j}^{m} \hat{N}_{k, j}^{(1)}\right\rangle, \\
& \tilde{w}_{p q_{1}}^{(2,1)}=\left\langle\beta_{p q_{1}}^{m}+\beta_{i q_{2}}^{m} \hat{N}_{i p q_{1}, q_{2}}^{(1)}\right\rangle .
\end{aligned}
$$

Given the first mechanical cell problem 47) at the order $\varepsilon^{-1}$

$$
\left(C_{p j k l}^{m} N_{k i q_{1}, l}^{(1)}\right)_{, j}+C_{p j i q_{1}, j}^{m}=0
$$

its weak form has expression

$$
\left\langle\left[\left(C_{p j k l}^{m} N_{k i q_{1}, l}^{(1)}\right)_{, j}+C_{p j i q_{1}, j}^{m}\right] \hat{N}_{p}^{(1)}\right\rangle=0
$$

with test function $\hat{N}_{p}^{(1)}$. Equation 110 can be written as

$$
\left\langle\left[C_{p j k l}^{m} N_{k i q_{1}, l}^{(1)}+C_{p j i q_{1}}^{m}\right] \hat{N}_{p, j}^{(1)}\right\rangle=0
$$

for divergence theorem and $\mathcal{Q}$-periodicity of micro constitutive tensors components and weight functions. By adding vanishing term 111 to equation 108a one obtains

$$
\hat{n}_{p q_{1}}^{(2)}=\left\langle\beta_{p q_{1}}^{m}-C_{p q_{1} i l}^{m} \hat{N}_{i, l}^{(1)}+C_{i j k l}^{m} N_{i p q_{1}, j}^{(1)} \hat{N}_{k, l}^{(1)}+C_{i l p q_{1}}^{m} \hat{N}_{i, l}^{(1)}\right\rangle=\left\langle\beta_{p q_{1}}^{m}+C_{i j k l}^{m} N_{i p q_{1}, j}^{(1)} \hat{N}_{k, l}^{(1)}\right\rangle .
$$

From cell problem 49, at the order $\varepsilon^{-1}$

$$
\left(C_{i j k l}^{m} \hat{N}_{k, l}^{(1)}\right)_{, j}-\beta_{i j, j}^{m}=0
$$

the following weak form can be derived

$$
\left\langle\left[\left(C_{i j k l}^{m} \hat{N}_{k, l}^{(1)}\right)_{, j}-\beta_{i j, j}^{m}\right] N_{i p q_{1}}^{(1)}\right\rangle=0,
$$


considering $N_{i p q_{1}}^{(1)}$ as a test function. Analogously to what done before, equation 114 can be written in the form

$$
\left\langle\left[C_{i j k l}^{m} \hat{N}_{k, l}^{(1)}-\beta_{i j}^{m}\right] N_{i p q_{1}, j}^{(1)}\right\rangle=0 .
$$

The sum of 115 and $108 \mathrm{~b}$ leads to

$$
\tilde{w}_{p q_{1}}^{(2,1)}=\left\langle\beta_{p q_{1}}^{m}+\beta_{i q_{2}}^{m} \hat{N}_{i p q_{1}, q_{2}}^{(1)}+C_{i j k l}^{m} \hat{N}_{k, l}^{(1)} N_{i p q_{1}, j}^{(1)}-\beta_{i j}^{m} N_{i p q_{1}, j}^{(1)}\right\rangle=\left\langle\beta_{p q_{1}}^{m}+C_{i j k l}^{m} \hat{N}_{k, l}^{(1)} N_{i p q_{1}, j}^{(1)}\right\rangle,
$$

from which equivalence $\hat{n}_{p q_{1}}^{(2)}=\tilde{w}_{p q_{1}}^{(2,1)}$ follows.

Proof of $\hat{m}^{(2,1)}=\hat{w}^{(2,1)}$

Constants $\hat{m}^{(2,1)}$ and $\hat{w}^{(2,1)}$ come from the known terms of cell problems (58) and 63), namely

$$
\begin{aligned}
& \hat{m}^{(2,1)}=\left\langle\psi^{m}+\alpha_{q_{1} q_{2}}^{m} \hat{N}_{q_{1}, q_{2}}^{(1)}\right\rangle, \\
& \hat{w}^{(2,1)}=\left\langle\psi^{m}+\beta_{q_{1} q_{2}}^{m} \tilde{N}_{q_{1}, q_{2}}^{(1)}\right\rangle .
\end{aligned}
$$

Second mechanical cell problem 48 at the order $\varepsilon^{-1}$ reads

$$
\left(C_{i j k l}^{m} \tilde{N}_{k, l}^{(1)}\right)_{, j}-\alpha_{i j, j}^{m}=0
$$

and its weak form, considering $\hat{N}_{i}^{(1)}$ as test function, is

$$
\left\langle\left[\left(C_{i j k l}^{m} \tilde{N}_{k, l}^{(1)}\right)_{, j}-\alpha_{i j, j}^{m}\right] \hat{N}_{i}^{(1)}\right\rangle=0 .
$$

Once again, equation 119 can be written as

$$
\left\langle\left[C_{i j k l}^{m} \tilde{N}_{k, l}^{(1)}-\alpha_{i j}^{m}\right] \hat{N}_{i, j}^{(1)}\right\rangle=0,
$$

exploiting divergence theorem and $\mathcal{Q}$-periodicity of perturbation functions and micro constitutive tensors. Adding term 120 to 117a), one obtains

$$
\hat{m}^{(2,1)}=\left\langle\psi^{m}+\alpha_{q_{1} q_{2}}^{m} \hat{N}_{q_{1}, q_{2}}^{(1)}+C_{i j k l}^{m} \tilde{N}_{k, l}^{(1)} \hat{N}_{i, j}^{(1)}-\alpha_{i j}^{m} \hat{N}_{i, j}^{(1)}\right\rangle=\left\langle\psi^{m}+C_{i j k l}^{m} \hat{N}_{i, j}^{(1)} \tilde{N}_{k, l}^{(1)}\right\rangle .
$$

The weak form of cell problem 49 at the order $\varepsilon^{-1}$, expressed as

$$
\left(C_{i j k l}^{m} \hat{N}_{k, l}^{(1)}\right)_{, j}-\beta_{i j, j}^{m}=0
$$

has the form

$$
\left\langle\left[\left(C_{i j k l}^{m} \hat{N}_{k, l}^{(1)}\right)_{, j}-\beta_{i j, j}^{m}\right] \tilde{N}_{i}^{(1)}\right\rangle=0,
$$

with test function $\tilde{N}_{i}^{(1)}$. Equation 123 turns into

$$
\left\langle\left[C_{i j k l}^{m} \hat{N}_{k, l}^{(1)}-\beta_{i j}^{m}\right] \hat{N}_{i, j}^{(1)}\right\rangle=0
$$

for divergence theorem and $\mathcal{Q}$-periodicity of terms involved. The sum of vanishing term 124 and $117 \mathrm{~b}$ leads to

$$
\hat{w}^{(2,1)}=\left\langle\psi^{m}+\beta_{q_{1} q_{2}}^{m} \tilde{N}_{q_{1}, q_{2}}^{(1)}-\beta_{i j}^{m} \hat{N}_{i, j}^{(1)}+C_{i j k l}^{m} \hat{N}_{k, l}^{(1)} \hat{N}_{i, j}^{(1)}\right\rangle=\left\langle\psi^{m}+C_{i j k l}^{m} \hat{N}_{k, l}^{(1)} \hat{N}_{i, j}^{(1)}\right\rangle .
$$

Comparing 121 and 125, the equivalence between constants $\hat{m}^{(2,1)}$ and $\hat{w}^{(2,1)}$ trivially derives. 


\section{Appendix B. Fourth order approximation of dispersion functions for the equivalent thermo-diffusive medium}

Considering an angular coordinate $\phi=0$, sensitivities $\omega_{i}^{[n]}$, with $n=1, . ., 4$ and $i=1, \ldots, 6$ of table 1 have the closed form detailed below in terms of the overall constitutive tensors components relative to the homogenized thermo-diffusive medium. Such sensitivities are the solutions of the chain of $n$-ordered perturbation equations generated by the perturbative approximation described in Section 6.1 of the characteristic equation (91). Being $k_{1}=r \cos (\phi)=r$ and $k_{2}=r \sin (\phi)=0$, perturbation parameter is represented by the wave number $k_{1}$ and the fourth order approximated dispersion function $\omega\left(k_{1}\right)$ has the form

$$
\omega\left(k_{1}\right)=\omega^{[0]}+\omega^{[1]} k_{1}+\omega^{[2]} k_{1}^{2}+\omega^{[3]} k_{1}^{3}+\omega^{[4]} k_{1}^{4}+O\left(k_{1}^{5}\right)
$$

As expected, generating solutions at the order $k_{1}^{0}$ are all vanishing, namely $\omega_{1 \ldots 6}^{[0]}=0$ for $i=1, \ldots, 6$, meaning that dispersion curves are all acoustic branches departing from the origin. From perturbation problem $G^{[6]}(r=0)=0$ one derives the following sensitivities

$$
\begin{aligned}
& \omega_{1,2}^{[1]}=0, \\
& \omega_{3}^{[1]}=\frac{i \sqrt{\rho C_{1212}}}{\rho}, \\
& \omega_{5}^{[1]}=\frac{i \sqrt{\rho\left(p q-\psi^{2}\right)\left(C_{1111} p q-C_{1111} \psi^{2}+\alpha_{11}^{2} q+2 \alpha_{11} \beta_{11} \psi+\beta_{11}^{2} p\right)}}{\rho\left(p q-\psi^{2}\right)},
\end{aligned}
$$

where $\omega_{4}^{[1]}$ and $\omega_{6}^{[1]}$ are not explicitly written being the complex conjugate of sensitivities $\omega_{3}^{[1]}$ and $\omega_{5}^{[1]}$, respectively. Consistently, sensitivities $\omega_{i}^{[2]}$ have the form

$$
\begin{aligned}
\omega_{1}^{[2]} & =\frac{-p C_{1111} D_{11}-q C_{1111} K_{11}-D_{11} \alpha_{11}^{2}-K_{11} \beta_{11}^{2}+r_{1}}{2 C_{1111} p q-2 C_{1111} \psi^{2}+2 \alpha_{11}^{2} q+4 \alpha_{11} \beta_{11} \psi+2 \beta_{11}^{2} p}, \\
\omega_{2}^{[2]} & =\frac{-p C_{1111} D_{11}-q C_{1111} K_{11}-D_{11} \alpha_{11}^{2}-K_{11} \beta_{11}^{2}-r_{1}}{2 C_{1111} p q-2 C_{1111} \psi^{2}+2 \alpha_{11}^{2} q+4 \alpha_{11} \beta_{11} \psi+2 \beta_{11}^{2} p}, \\
\omega_{3}^{[2]} & =0 \\
\omega_{5}^{[2]} & =\frac{1}{2} \frac{\left(-D_{11} \alpha_{11}^{2}-K_{11} \beta_{11}^{2}\right) \psi^{2}-2 \psi \beta_{11}\left(D_{11} p+K_{11} q\right) \alpha_{11}-D_{11} \beta_{11}^{2} p^{2}-K_{11} \alpha_{11}^{2} q^{2}}{\left(p q-\psi^{2}\right)\left(\left(C_{1111} q+\beta_{11}^{2}\right) p-C_{1111} \psi^{2}+2 \beta_{11} \alpha_{11} \psi+\alpha_{11}^{2} q\right)} .
\end{aligned}
$$

Sensitivities $\omega_{i}^{[3]}$ are expressed as

$$
\begin{aligned}
& \omega_{1}^{[3]}=\omega_{2}^{[3]}=\omega_{3}^{[3]}=0, \\
& \omega_{5}^{[3]}=\left(\alpha_{11}^{4} a_{40}+\alpha_{11}^{3} \beta_{11} a_{31}+\alpha_{11}^{2} \beta_{11}^{2} a_{22}+\alpha_{11} \beta_{11}^{3} a_{13}+\beta_{11}^{4} a_{04}+\alpha_{11}^{2} a_{20}+\alpha_{11} \beta_{11} a_{11}+\beta_{11}^{2} a_{02}\right) / s_{1},
\end{aligned}
$$

and sensitivities $\omega_{i}^{[4]}$ result

$$
\begin{aligned}
\omega_{1}^{[4]} & =\left(D_{11}^{4} b_{40}+D_{11}^{3} K_{11} b_{31}+D_{11}^{2} K_{11}^{2} b_{22}+D_{11} K_{11}^{3} b_{13}+K_{11}^{4} b_{04}+D_{11}^{3} b_{30}+\right. \\
& \left.+D_{11}^{2} K_{11} b_{21}+D_{11} K_{11}^{2} b_{12}+K_{11}^{3} b_{03}\right) / s_{2}, \\
\omega_{2}^{[4]} & =\left(D_{11}^{4} c_{40}+D_{11}^{3} K_{11} c_{31}+D_{11}^{2} K_{11}^{2} c_{22}+D_{11} K 11^{3} c_{13}+K_{11}^{4} c_{04}+D_{11}^{3} c_{30}+\right. \\
& \left.+D_{11}^{2} K_{11} c_{21}+D_{11} K_{11}^{2} c_{12}+K_{11}^{3} c_{30}\right) / s_{3}, \\
\omega_{3}^{[4]} & =0 \\
\omega_{5}^{[4]} & =\left(\alpha_{11}^{6} d_{60}+\alpha_{11}^{5} \beta_{11} d_{51}+\alpha_{11}^{4} \beta_{11}^{2} d_{42}+\alpha_{11}^{2} \beta_{11}^{4} d_{24}+\alpha_{11} \beta_{11}^{5} d_{15}+\beta_{11}^{6} d_{06}+\alpha_{11}^{4} d_{40}+\right. \\
& \left.+\alpha_{11}^{3} \beta_{11} d_{31}+\alpha_{11}^{2} \beta_{11}^{2} d_{22}+\alpha_{11} \beta_{11}^{3} d_{13}+\beta_{11}^{4} d_{04}+\alpha_{11}^{2} d_{20}+\alpha_{11} \beta_{11} d_{11}+\beta_{11}^{2} d_{02}\right) / s_{4} .
\end{aligned}
$$

Coefficients $r_{i}, s_{i}, a_{i j}, b_{i j}, c_{i j}$, and $d_{i j}$ of formulas 128, (129), and 130 are made explicit in Appendix C. 


\section{Appendix C. Coefficients involved in the perturbative approxima- tion of dispersion functions}

Coefficient $r_{1}$ of equation 128 reads

$$
\begin{aligned}
r_{1} & =\left(\left(C_{11} p+\alpha_{11}^{2}\right)^{2} D_{11}^{2}-2 K_{11}\left(\left(C_{1111} q-\beta_{11}^{2}\right) \alpha_{11}^{2}+4 \psi C_{1111} \beta_{11} \alpha_{11}+\left(\beta_{11}^{2} p+\right.\right.\right. \\
& \left.\left.\left.+C_{1111}\left(p q-2 \psi^{2}\right)\right) C_{1111}\right) D_{11}+K_{11}^{2}\left(C_{1111} q+\beta_{11}^{2}\right)^{2}\right)^{1 / 2} .
\end{aligned}
$$

Coefficients related to sensitivity $\omega_{5}^{[3]}$ of equation 129 have the form

$$
\begin{aligned}
a_{40} & =\rho\left(4 D_{11}^{2} p \psi^{2} q-3 D_{11}^{2} \psi^{4}+2 D_{11} K_{11} \psi^{2} q^{2}+K_{11}^{2} q^{4}\right), \\
a_{31} & =4 \rho \psi\left(2 D_{11}^{2} p^{2} q-D_{11}^{2} p \psi^{2}-D_{11} K_{11} p q^{2}+3 D_{11} K_{11} \psi^{2} q+K_{11}^{2} q^{3}\right), \\
a_{22} & =4\left(p q^{3} K_{11}^{2}+\left(-3 / 2 p^{2} D_{11} K_{11}+1 / 2 K_{11}^{2} \psi^{2}\right) q^{2}+p D_{11}\left(D_{11} p^{2}+2 K_{11} \psi^{2}\right) q+\right. \\
& \left.+1 / 2 D_{11} \psi^{2}\left(D_{11} p^{2}+5 K_{11} \psi^{2}\right)\right) \rho, \\
a_{13} & =4\left(\left(2 p q^{2}-\psi^{2} q\right) K_{11}^{2}-p K_{11}\left(p q-3 \psi^{2}\right) D_{11}+p^{3} D_{11}^{2}\right) \psi \rho, \\
a_{04} & =\left(-3 K_{11}^{2} \psi^{4}+\left(2 D_{11} K_{11} p^{2}+4 K_{11}^{2} p q\right) \psi^{2}+p^{4} D_{11}^{2}\right) \rho, \\
a_{20} & =4\left(p q-\psi^{2}\right)\left(D_{11}^{2} p \psi^{2}+2 D_{11} K_{11} \psi^{2} q+K_{11}^{2} q^{3}\right) \rho C_{1111}, \\
a_{11} & =8\left(p q-\psi^{2}\right)\left(D_{11}^{2} p^{2}+K_{11}\left(p q+\psi^{2}\right) D_{11}+K_{11}^{2} q^{2}\right) \psi \rho C_{1111}, \\
a_{02} & =4\left(p q-\psi^{2}\right)\left(D_{11}^{2} p^{3}+2 D_{11} K_{11} p \psi^{2}+K_{11}^{2} \psi^{2} q\right) \rho C_{1111}, \\
s_{1} & =8 i \sqrt{\rho}\left(p q-\psi^{2}\right)^{3 / 2}\left(\left(C_{1111} p+\alpha_{11}^{2}\right) q+\beta_{11}^{2} p-C_{1111} \psi^{2}+2 \beta_{11} \alpha_{11} \psi\right)^{5 / 2} .
\end{aligned}
$$

Sensitivity $\omega_{1}^{[4]}$ in equation 130 has coefficients

$$
\begin{aligned}
b_{40}= & 4\left(C_{1111} p+\alpha_{11}^{2}\right)^{3}\left(\alpha_{11} \psi+\beta_{11} p\right)^{2} C_{1212} \rho \\
b_{31}= & -4\left(2 q \beta_{11} \alpha_{11}^{4}-q \psi C_{1111} \alpha_{11}^{3}+\left(-2 p \beta_{11}^{3}+C_{1111}\left(3 p q+4 \psi^{2}\right) \beta_{11}\right) \alpha_{11}^{2}+\right. \\
- & \left.\left(-9 \beta_{11}^{2} p+C_{1111}\left(p q+3 \psi^{2}\right)\right) \psi C_{1111} \alpha_{11}+\beta_{11}\left(\beta_{11}^{2} p+C_{1111}\left(p q-5 \psi^{2}\right)\right) p C_{1111}\right) \\
& \left(C_{1111} p+\alpha_{11}^{2}\right)\left(\alpha_{11} \psi+\beta_{11} p\right) C_{1212} \rho \\
b_{22}= & -4\left(\alpha_{11}^{4} q^{2}-2 \alpha_{11}^{3} q \psi \beta_{11}+\left(\left(-4 p q-2 \psi^{2}\right) \beta_{11}^{2}+q C_{1111}\left(p q+5 \psi^{2}\right)\right) \alpha_{11}^{2}+\right. \\
+ & \left.8 \beta_{11}\left(-1 / 4 \beta_{11}^{2} p+C_{1111}\left(p q+1 / 2 \psi^{2}\right)\right) \psi \alpha_{11}+\beta_{11}^{2} p\left(\beta_{11}^{2} p+C_{1111}\left(p q+5 \psi^{2}\right)\right)\right) \\
& \left(C_{1111} \psi-\alpha_{11} \beta_{11}\right)^{2} C_{1212} \rho \\
b_{13}= & -4\left(2 \alpha_{11} \beta_{11}^{4} p-C_{1111} \beta_{11}^{3} p \psi+\left(\alpha_{11}\left(3 p q+4 \psi^{2}\right) C_{1111}-2 \alpha_{11}^{3} q\right) \beta_{11}^{2}+\right. \\
- & \left.\left(C_{1111}\left(p q+3 \psi^{2}\right)-9 \alpha_{11}^{2} q\right) C_{1111} \psi \beta_{11}+\left(C_{1111}\left(p q-5 \psi^{2}\right)+\alpha_{11}^{2} q\right) q C_{1111} \alpha_{11}\right) \\
& C_{1212}\left(C_{1111} q+\beta_{11}^{2}\right) \rho\left(\alpha_{11} q+\beta_{11} \psi\right), \\
b_{04}= & 4\left(C_{1111} q+\beta_{11}^{2}\right)^{3}\left(\alpha_{11} q+\beta_{11} \psi\right)^{2} C_{1212} \rho, \\
b_{30}= & -4 r_{2}\left(C_{1111} p+\alpha_{11}^{2}\right)^{2}\left(\alpha_{11} \psi+\beta_{11} p\right)^{2} C_{1212} \rho, \\
b_{21}= & -4\left(\alpha_{11} \psi+\beta_{11} p\right)\left(2 C_{1111} \alpha_{11} p q+C_{1111} \alpha_{11} \psi^{2}+3 C_{1111} \beta_{11} p \psi+2 \alpha_{11}^{3} q+\right. \\
+ & \left.\alpha_{11}^{2} \beta_{11} \psi-\alpha_{11} \beta_{11}^{2} p\right) r_{2}\left(C_{1111} \psi-\alpha_{11} \beta_{11}\right) C_{1212} \rho, \\
b_{12}= & -4\left(3 C_{1111} \alpha_{11} \psi q+2 C_{1111} \beta_{11} p q+C_{1111} \beta_{11} \psi^{2}-\alpha_{11}^{2} \beta_{11} q+\alpha_{11} \beta_{11}^{2} \psi+2 \beta_{11}^{3} p\right) \\
& \left(C_{1111} \psi-\alpha_{11} \beta_{11}\right) r_{1}\left(\alpha_{11} q+\beta_{11} \psi\right) C_{1212} \rho, \\
b_{03}= & -4\left(C_{1111} q+\beta_{11}^{2}\right)^{2} r_{1}\left(\alpha_{11} q+\beta_{11} \psi\right)^{2} C_{1212} \rho, \\
s_{2}= & 8 C_{1212}\left(\left(C_{1111} p+\alpha_{11}^{2}\right) q+\beta_{11}^{2} p-C_{1111} \psi^{2}+2 \beta_{11} \alpha_{11} \psi\right)^{4} r_{2}, \\
r_{2}= & \left(\left(C_{1111} p+\alpha_{11}^{2}\right)^{2} D 11^{2}-2 K_{11}\left(\left(C_{1111} q-\beta_{11}^{2}\right) \alpha_{11}^{2}+4 \psi C_{1111} \beta_{11} \alpha_{11}+\right.\right. \\
+ & \left.\left.\left(\beta_{11}^{2} p+C_{1111}\left(p q-2 \psi^{2}\right)\right) C_{1111}\right) D_{11}+K_{11}^{2}\left(C_{1111} q+\beta_{11}^{2}\right)^{2}\right)^{1 / 2} . \\
= &
\end{aligned}
$$


Coefficients of sensitivity $\omega_{2}^{[4]}$ in equation 130 have the following expression

$$
\begin{aligned}
c_{40}= & -4\left(C_{1111} p+\alpha_{11}^{2}\right)^{3}\left(\alpha_{11} \psi+\beta_{11} p\right)^{2} C_{1212} \rho \\
c_{31}= & 4\left(2 q \beta_{11} \alpha_{11}^{4}-q \psi C_{1111} \alpha_{11}^{3}+\left(-2 p \beta_{11}^{3}+C_{1111}\left(3 p q+4 \psi^{2}\right) \beta_{11}\right) \alpha_{11}^{2}+\right. \\
- & \left.\left(-9 \beta_{11}^{2} p+C_{1111}\left(p q+3 \psi^{2}\right)\right) \psi C_{1111} \alpha_{11}+\beta_{11}\left(\beta_{11}^{2} p+C_{1111}\left(p q-5 \psi^{2}\right)\right) p C_{1111}\right) \\
& \left(C_{1111} p+\alpha_{11}^{2}\right)\left(\alpha_{11} \psi+\beta_{11} p\right) C_{1212} \rho, \\
c_{22}= & -4\left(\alpha_{11}^{4} q^{2}-2 \alpha_{11}^{3} q \psi \beta_{11}+\left(\left(-4 p q-2 \psi^{2}\right) \beta_{11}^{2}+q C_{1111}\left(p q+5 \psi^{2}\right)\right) \alpha_{11}^{2}+\right. \\
+ & \left.8 \beta_{11}\left(-1 / 4 \beta_{11}^{2} p+C_{1111}\left(p q+1 / 2 \psi^{2}\right)\right) \psi \alpha_{11}+\beta_{11}^{2} p\left(\beta_{11}^{2} p+C_{1111}\left(p q+5 \psi^{2}\right)\right)\right) \\
& \left(C_{1111} \psi-\alpha_{11} \beta_{11}\right)^{2} C_{1212} \rho \\
= & 4\left(-C_{1111} \beta_{11}^{3} p \psi+2 \alpha_{11} \beta_{11}^{4} p+\left(\alpha_{11}\left(3 p q+4 \psi^{2}\right) C_{1111}-2 \alpha_{11}^{3} q\right) \beta_{11}^{2}+\right. \\
c_{13} & \left.\left(C_{1111}\left(p q+3 \psi^{2}\right)-9 \alpha_{11}^{2} q\right) C_{1111} \psi \beta_{11}+\left(C_{1111}\left(p q-5 \psi^{2}\right)+\alpha_{11}^{2} q\right) q C_{1111} \alpha_{11}\right) \\
& C_{1212}\left(C_{1111} q+\beta_{11}^{2}\right) \rho\left(\alpha_{11} q+\beta_{11} \psi\right), \\
c_{40}= & -4\left(C_{1111} q+\beta_{11}^{2}\right)^{3}\left(\alpha_{11} q+\beta_{11} \psi\right)^{2} C_{1212} \rho, \\
c_{30}= & -4 r_{2}\left(C_{1111} p+\alpha_{11}^{2}\right)^{2}\left(\alpha_{11} \psi+\beta_{11} p\right)^{2} C_{1212} \rho, \\
c_{21}= & -4\left(\alpha_{11} \psi+\beta_{11} p\right)\left(2 C_{1111} \alpha_{11} p q+C_{1111} \alpha_{11} \psi^{2}+3 C_{1111} \beta_{11} p \psi+2 \alpha_{11}^{3} q+\right. \\
+ & \left.\alpha_{11}^{2} \beta_{11} \psi-\alpha_{11} \beta_{11}^{2} p\right) r_{2}\left(C_{1111} \psi-\alpha_{11} \beta_{11}\right) C_{1212} \rho, \\
c_{12}= & -4\left(3 C_{1111} \alpha_{11} \psi q+2 C_{1111} \beta_{11} p q+C_{1111} \beta_{11} \psi^{2}-\alpha_{11}^{2} \beta_{11} q+\alpha_{11} \beta_{11}^{2} \psi+2 \beta_{11}^{3} p\right) \\
& \left(C_{1111} \psi-\alpha_{11} \beta_{11}\right) r_{2}\left(\alpha_{11} q+\beta_{11} \psi\right) C_{1212} \rho, \\
c_{03}= & -4\left(C_{1111} q+\beta_{11}^{2}\right)^{2} r_{1}\left(\alpha_{11} q+\beta_{11} \psi\right)^{2} \rho, \\
s_{3}= & 8 C_{1212}\left(\left(C_{1111} p+\alpha_{11}^{2}\right) q+\beta_{11}^{2} p-C_{1111} \psi^{2}+2 \beta_{11} \alpha_{11} \psi\right)^{4} r_{2}, \\
&
\end{aligned}
$$

while coefficients of sensitivity $\omega_{5}^{[4]}$ in equation $130 \mathrm{read}$

$$
\begin{aligned}
& d_{60}=\rho D_{11}^{3} \psi^{2} \\
& d_{51}=2 \rho D_{11}^{2} \psi\left(D_{11} p-K_{11} q\right) \\
& d_{42}=\rho\left(D_{11}^{2} p^{2}+\left(-2 p q-\psi^{2}\right) K_{11} D_{11}+K_{11}^{2} q^{2}\right) D_{11}, \\
& d_{24}=\rho\left(D_{11}^{2} p^{2}+\left(-2 p q-\psi^{2}\right) K_{11} D_{11}+K_{11}^{2} q^{2}\right) K_{11}, \\
& d_{15}=-2 \rho K_{11}^{2}\left(D_{11} p-K_{11} q\right) \psi \\
& d_{06}=\rho K_{11}^{3} \psi^{2} \\
& d_{40}=2 \rho D_{11}^{2} \psi^{2} C_{1111}\left(D_{11} p+K_{11} q\right) \\
& d_{31}=4 \rho C_{111} D_{11} \psi\left(D_{11}^{2} p^{2}-K_{11}^{2} q^{2}\right) \\
& d_{22}=2 \rho\left(D_{11} p+K_{11} q\right)\left(D_{11}^{2} p^{2}-2 K_{11}\left(p q+\psi^{2}\right) D_{11}+K_{11}^{2} q^{2}\right) C_{1111}, \\
& d_{13}=-4 \rho C_{1111} K_{11}\left(D_{11}^{2} p^{2}-K_{11}^{2} q^{2}\right) \psi \\
& d_{04}=2 \rho K_{11}^{2} \psi^{2} C_{1111}\left(D_{11} p+K_{11} q\right) \\
& d_{20}=\rho\left(p^{2} D_{11}^{3} \psi^{2}+\left(2 p \psi^{2} q+\psi^{4}\right) K_{11} D_{11}^{2}+3 q^{2} D_{11} K_{11}^{2} \psi^{2}+q^{4} K_{11}^{3}\right) C_{1111}^{2}, \\
& d_{11}=2 \rho C_{1111}^{2}\left(D_{11} p+K_{11} q\right)\left(D_{11}^{2} p^{2}+2 D_{11} K_{11} \psi^{2}+K_{11}^{2} q^{2}\right) \psi \\
& d_{02}=\rho\left(p^{4} D_{11}^{3}+3 p^{2} D_{11}^{2} K_{11} \psi^{2}+\left(2 p \psi^{2} q+\psi^{4}\right) K_{11}^{2} D_{11}+q^{2} K_{11}^{3} \psi^{2}\right) C_{1111}^{2}, \\
& s_{4}=2\left(\left(C_{1111} p+\alpha_{11}^{2}\right) q+\beta_{11}^{2} p-C_{1111} \psi^{2}+2 \beta_{11} \alpha_{11} \psi\right)^{4} .
\end{aligned}
$$




\section{Appendix D. Tensorial fashion for constitutive equations of thermo- diffusive material}

In a 2-D setting, linear constitutive relations $\sqrt{1 \mathrm{a}})-(1 \mathrm{c})$ for thermo-diffusive materials can rigorously be written in a tensorial fashion as done in Mehrabadi and Cowin (1990). They read

$$
\begin{aligned}
&\left(\begin{array}{c}
\sigma_{11} \\
\sigma_{22} \\
\sqrt{2} \sigma_{12}
\end{array}\right)=\left(\begin{array}{ccc}
C_{1111}^{m} & C_{1122}^{m} & \sqrt{2} C_{1112}^{m} \\
C_{2211}^{m} & C_{2222}^{m} & \sqrt{2} C_{2212}^{m} \\
\sqrt{2} C_{1211}^{m} & \sqrt{2} C_{1222}^{m} & 2 C_{1212}^{m}
\end{array}\right)\left(\begin{array}{c}
u_{1,1} \\
u_{2,2} \\
\frac{\sqrt{2}}{2}\left(u_{1,2}+u_{2,1}\right)
\end{array}\right)+ \\
&-\left(\begin{array}{c}
\alpha_{11}^{m} \\
\alpha_{22}^{m} \\
\sqrt{2} \alpha_{12}^{m}
\end{array}\right) \theta-\left(\begin{array}{c}
\beta_{11}^{m} \\
\beta_{22}^{m} \\
\sqrt{2} \beta_{12}^{m}
\end{array}\right) \eta, \\
&\left(\begin{array}{c}
q_{1} \\
q_{2}
\end{array}\right)=-\left(\begin{array}{cc}
K_{11}^{m} & K_{12}^{m} \\
K_{21}^{m} & K_{22}^{m}
\end{array}\right)\left(\begin{array}{c}
\theta_{, 1} \\
\theta_{, 2}
\end{array}\right), \\
&\left(\begin{array}{c}
j_{1} \\
j_{2}
\end{array}\right)=-\left(\begin{array}{ll}
D_{11}^{m} & D_{12}^{m} \\
D_{21}^{m} & D_{22}^{m}
\end{array}\right)\left(\begin{array}{c}
\eta_{, 1} \\
\eta_{, 2}
\end{array}\right) .
\end{aligned}
$$

\section{Appendix E. Frequency band structure of heterogeneous periodic thermo-diffusive material: finite element formulation}

Constitutive relations (1a)- $1 \mathrm{c}$ ) for thermo-diffusive materials in indicial form read

$$
\begin{aligned}
& \sigma_{i j}=C_{i j k l}^{m} u_{k, l}-\alpha_{i j}^{m} \theta-\beta_{i j}^{m} \eta, \\
& q_{i}=-K_{i j}^{m} \theta_{, j}, \\
& j_{i}=-D_{i j}^{m} \eta_{, j} .
\end{aligned}
$$

Denoting with $\mathbf{b}$ the body force vector, with $r$ the heat source term, and with $s$ the mass source term, stress tensor $\boldsymbol{\sigma}$, heat flux vector $\mathbf{q}$, and mass flux vector $\mathbf{j}$ satisfy local balance equations (2a)-(2c), here written in the form

$$
\begin{aligned}
& \left(C_{i j k l}^{m} u_{k, l}\right)_{, j}-\left(\alpha_{i j}^{m} \theta\right)_{, j}-\left(\beta_{i j}^{m} \eta\right)_{, j}+b_{i}=\rho^{m} \ddot{u}_{i}, \\
& \left(K_{i j}^{m} \theta_{, j}\right)_{, i}-\alpha_{i j}^{m} \dot{u}_{i, j}-\psi^{m} \dot{\eta}+r=p^{m} \dot{\theta}, \\
& \left(D_{i j}^{m} \eta_{, j}\right)_{, i}-\beta_{i j}^{m} \dot{u}_{i, j}-\psi^{m} \dot{\theta}+s=q^{m} \dot{\theta} .
\end{aligned}
$$

Dirichlet and Neumann part of the boundary $\partial \Omega$ of domain $\Omega$, denoted respectively as $\left\{\partial \Omega_{\mathbf{u}}, \partial \Omega_{\theta}, \partial \Omega_{\eta}\right\}$ and $\left\{\partial \Omega_{\boldsymbol{\sigma}}, \partial \Omega_{\mathbf{q}}, \partial \Omega_{\mathbf{j}}\right\}$, are such that $\partial \Omega=\partial \Omega_{\mathbf{u}} \cup \partial \Omega_{\boldsymbol{\sigma}}=\partial \Omega_{\theta} \cup \partial \Omega_{\mathbf{q}}=\partial \Omega_{\eta} \cup \partial \Omega_{\mathbf{j}}$ and $\partial \Omega_{\mathbf{u}} \cap \partial \Omega_{\boldsymbol{\sigma}}=\partial \Omega_{\theta} \cap \partial \Omega_{\mathbf{q}}=$ $\partial \Omega_{\eta} \cap \partial \Omega_{\mathbf{j}}=\emptyset$. Micro fields satisfy boundary conditions

$$
\begin{aligned}
& \left\{\begin{array}{ll}
u_{i}=\bar{u}_{i} & \text { on } \partial \Omega_{\mathbf{u}} \\
\sigma_{i j} n_{j}=\bar{t}_{i} & \text { on } \partial \Omega_{\boldsymbol{\sigma}}
\end{array}, \quad\left\{\begin{array}{ll}
\theta=\bar{\theta} & \text { on } \partial \Omega_{\theta} \\
q_{i} n_{i}=\bar{q} & \text { on } \partial \Omega_{\mathbf{q}}
\end{array},\right.\right. \\
& \begin{cases}\eta=\bar{\eta} & \text { on } \partial \Omega_{\eta} \\
j_{i} n_{i}=\bar{j} & \text { on } \partial \Omega_{\mathbf{j}}\end{cases}
\end{aligned}
$$

where $\bar{t}_{i}, \bar{q}$ and $\bar{j}$ are the prescribed values of tractions, heat flux, and mass flux, respectively, and $\mathbf{n}$ is the outward normal to the boundary of the domain $\partial \Omega$. Taking into account boundary conditions (139), weak form of local balance equations (138) reads

$$
\begin{aligned}
& \int_{\Omega}\left(C_{i j k l}^{m} u_{k, l}-\alpha_{i j}^{m} \theta-\beta_{i j}^{m} \eta\right) \varphi_{u_{i, j}} d V-\int_{\partial \Omega_{\sigma}} \bar{t}_{i} \varphi_{u_{i}} d S-\int_{\Omega} b_{i} \varphi_{u_{i}} d V+ \\
& \int_{\Omega} \rho^{m} \ddot{u}_{i} \varphi_{u_{i}} d V=0 \quad \forall \varphi_{u_{i}} \text { s.t. } \varphi_{u_{i}}=0 \quad \text { on } \partial \Omega_{\mathbf{u}},
\end{aligned}
$$




$$
\begin{aligned}
& \int_{\Omega}\left(K_{i j}^{m} \theta_{, j}\right) \varphi_{\theta, i} d V+\int_{\partial \Omega_{\mathbf{q}}} \bar{q} \varphi_{\theta} d S+\int_{\Omega}\left(\alpha_{i j}^{m} \dot{u}_{i, j}+\psi^{m} \dot{\eta}-r\right) \varphi_{\theta} d V+ \\
& \int_{\Omega} p^{m} \dot{\theta} \varphi_{\theta} d V=0 \quad \forall \varphi_{\theta} \text { s.t. } \varphi_{\theta}=0 \quad \text { on } \partial \Omega_{\theta}, \\
& \int_{\Omega}\left(D_{i j}^{m} \eta_{, j}\right) \varphi_{\eta, i} d V+\int_{\partial \Omega_{\mathbf{j}}} \bar{j} \varphi_{\eta} d S+\int_{\Omega}\left(\beta_{i j}^{m} \dot{u}_{i, j}+\psi^{m} \dot{\theta}-s\right) \varphi_{\eta} d V+ \\
& \int_{\Omega} q^{m} \dot{\eta} \varphi_{\eta} d V=0 \quad \forall \varphi_{\eta} \text { s.t. } \varphi_{\eta}=0 \quad \text { on } \partial \Omega_{\eta},
\end{aligned}
$$

with $\varphi_{u_{i}}, \varphi_{\theta}$ and $\varphi_{\eta}$ test functions. Micro fields $\mathbf{u}(\mathbf{x}, t), \theta(\mathbf{x}, t)$, and $\eta(\mathbf{x}, t)$ are approximated by a linear combination of shape functions $\mathbf{N}(\mathbf{x})$ and nodal unknowns $\mathbf{u}(t), \boldsymbol{\theta}(t)$, and $\boldsymbol{\eta}(t)$, as usual in a finite element discretization, and read

$$
u_{i}(\mathbf{x}, t)=\sum_{j=1}^{N_{h}} N_{j}(\mathbf{x}) u_{i_{j}}(t), \quad \theta(\mathbf{x}, t)=\sum_{j=1}^{N_{h}} N_{j}(\mathbf{x}) \theta_{j}(t), \quad \eta(\mathbf{x}, t)=\sum_{j=1}^{N_{h}} N_{j}(\mathbf{x}) \eta_{j}(t),
$$

and the very same discretization is performed for test functions, with nodal unknowns $\boldsymbol{\delta} \mathbf{u}(t), \boldsymbol{\delta} \boldsymbol{\theta}(t)$, and $\boldsymbol{\delta} \boldsymbol{\eta}(t)$

$$
\varphi_{u_{i}}(\mathbf{x}, t)=\sum_{j=1}^{N_{h}} N_{j}(\mathbf{x}) \delta u_{i_{j}}(t), \quad \varphi_{\theta}(\mathbf{x}, t)=\sum_{j=1}^{N_{h}} N_{j}(\mathbf{x}) \delta \theta_{j}(t), \quad \varphi_{\eta}(\mathbf{x}, t)=\sum_{j=1}^{N_{h}} N_{j}(\mathbf{x}) \delta \eta_{j}(t) .
$$

In equations (143) and (144), $N_{h}$ represents the finite dimension of the space $V_{h}$ for which $\left\{N_{j} \mid j=1,2, \ldots, N_{h}\right\}$ is a basis. In a two dimensional setting, denoting with $\mathbf{N}_{\mathbf{u}}, \mathbf{N}_{\theta}$, and $\mathbf{N}_{\eta}$ matrices collecting shape functions of the single finite element $e$ with $N_{N \text { nod }}$ the number of element nodes, one has

$$
\begin{aligned}
& \mathbf{N}_{\mathbf{u}}=\left[\begin{array}{ccccccc}
N_{1} & 0 & N_{2} & 0 & \ldots & N_{\text {Nnod }} & 0 \\
0 & N_{1} & 0 & N_{2} & \ldots & 0 & N_{\text {Nnod }}
\end{array}\right], \\
& \mathbf{N}_{\theta}=\mathbf{N}_{\eta}=\left[\begin{array}{lllll}
N_{1} & N_{2} & \ldots & N_{\text {Nnod }}
\end{array}\right],
\end{aligned}
$$

and denoting with $\mathbf{D}_{\mathbf{u}}, \mathbf{D}_{\theta}$, and $\mathbf{D}_{\eta}$ differential matrices

$$
\mathbf{D}_{\mathbf{u}}=\left[\begin{array}{cc}
\partial / \partial x_{1} & 0 \\
0 & \partial / \partial x_{2} \\
\partial / \partial x_{2} & \partial / \partial x_{1}
\end{array}\right], \quad \mathbf{D}_{\theta}=\mathbf{D}_{\eta}=\left[\begin{array}{c}
\partial / \partial x_{1} \\
\partial / \partial x_{2}
\end{array}\right]
$$

one defines $\mathbf{B}_{\mathbf{u}}=\mathbf{D}_{\mathbf{u}} \mathbf{N}_{\mathbf{u}}, \mathbf{B}_{\theta}=\mathbf{D}_{\theta} \mathbf{N}_{\theta}$, and $\mathbf{B}_{\eta}=\mathbf{D}_{\eta} \mathbf{N}_{\eta}$. Weak form 142 can therefore be written in matrix notation over each element domain $\Omega_{e}$ as

$$
\begin{aligned}
& \boldsymbol{\delta} \mathbf{u}^{T} \int_{\Omega_{e}} \mathbf{B}_{\mathbf{u}}^{T} \mathbf{C}^{m} \mathbf{B}_{\mathbf{u}} d V \mathbf{u}-\boldsymbol{\delta} \mathbf{u}^{T} \int_{\Omega_{e}} \mathbf{B}_{\mathbf{u}}^{T} \boldsymbol{\alpha}^{m} \mathbf{N}_{\theta} d V \boldsymbol{\theta}-\boldsymbol{\delta} \mathbf{u}^{T} \int_{\Omega_{e}} \mathbf{B}_{\mathbf{u}}^{T} \boldsymbol{\beta}^{m} \mathbf{N}_{\eta} d V \boldsymbol{\eta}+ \\
& -\boldsymbol{\delta} \mathbf{u}^{T} \int_{\partial \Omega_{e_{\boldsymbol{\sigma}}}} \mathbf{N}_{\mathbf{u}}^{T} \overline{\mathbf{t}} d S-\boldsymbol{\delta} \mathbf{u}^{T} \int_{\Omega_{e}} \mathbf{N}_{\mathbf{u}}^{T} \mathbf{b} d V+\boldsymbol{\delta} \mathbf{u}^{T} \int_{\Omega_{e}} \mathbf{N}_{\mathbf{u}}^{T} \rho^{m} \mathbf{N}_{\mathbf{u}} d V \ddot{\mathbf{u}}=0 \quad \forall \boldsymbol{\delta} \mathbf{u}, \\
& \boldsymbol{\delta} \boldsymbol{\theta}^{T} \int_{\Omega_{e}} \mathbf{B}_{\theta}^{T} \mathbf{K}^{m} \mathbf{B}_{\theta} d V \boldsymbol{\theta}+\boldsymbol{\delta} \boldsymbol{\theta}^{T} \int_{\Omega_{e}} \mathbf{N}_{\theta}^{T} \boldsymbol{\alpha}^{m} \mathbf{B}_{\mathbf{u}} d V \dot{\mathbf{u}}+\boldsymbol{\delta} \boldsymbol{\theta}^{T} \int_{\Omega_{e}} \mathbf{N}_{\theta}^{T} \psi^{m} \mathbf{N}_{\eta} d V \dot{\boldsymbol{\eta}}+ \\
& \boldsymbol{\delta} \boldsymbol{\theta}^{T} \int_{\partial \Omega_{e_{\mathbf{q}}}} \mathbf{N}_{\boldsymbol{\theta}}^{T} \bar{q} d S-\boldsymbol{\delta} \boldsymbol{\theta}^{T} \int_{\Omega_{e}} \mathbf{N}_{\theta}^{T} r d V+\boldsymbol{\delta} \boldsymbol{\theta}^{T} \int_{\Omega_{e}} \mathbf{N}_{\theta}^{T} p^{m} \mathbf{N}_{\theta} d V \dot{\boldsymbol{\theta}}=0 \quad \forall \boldsymbol{\delta} \boldsymbol{\theta}, \\
& \boldsymbol{\delta} \boldsymbol{\eta}^{T} \int_{\Omega_{e}} \mathbf{B}_{\eta}^{T} \mathbf{D}^{m} \mathbf{B}_{\eta} d V \boldsymbol{\eta}+\boldsymbol{\delta} \boldsymbol{\eta}^{T} \int_{\Omega_{e}} \mathbf{N}_{\eta}^{T} \boldsymbol{\beta}^{m} \mathbf{B}_{\mathbf{u}} d V \dot{\mathbf{u}}+\boldsymbol{\delta} \boldsymbol{\eta}^{T} \int_{\Omega_{e}} \mathbf{N}_{\eta}^{T} \psi^{m} \mathbf{N}_{\theta} d V \dot{\boldsymbol{\theta}}+ \\
& \boldsymbol{\delta} \boldsymbol{\eta}^{T} \int_{\partial \Omega_{e_{\mathbf{j}}}} \mathbf{N}_{\boldsymbol{\eta}}^{T} \bar{j} d S-\boldsymbol{\delta} \boldsymbol{\eta}^{T} \int_{\Omega_{e}} \mathbf{N}_{\eta}^{T} s d V+\boldsymbol{\delta} \boldsymbol{\eta}^{T} \int_{\Omega_{e}} \mathbf{N}_{\eta}^{T} q^{m} \mathbf{N}_{\eta} d V \dot{\boldsymbol{\eta}}=0 \quad \forall \boldsymbol{\delta} \boldsymbol{\eta},
\end{aligned}
$$


where symbols $\mathbf{C}^{m}, \mathbf{K}^{m}, \mathbf{D}^{m}, \boldsymbol{\alpha}^{m}$, and $\boldsymbol{\beta}^{m}$, denote the matrix form of the corresponding constitutive tensors $\mathfrak{C}^{m}, \boldsymbol{K}^{m}, \boldsymbol{D}^{m}, \boldsymbol{\alpha}^{m}$, and $\boldsymbol{\beta}^{m}$. Elemental stiffness matrices are defined in the following way

$$
\begin{aligned}
\mathbf{K}_{\mathbf{u u}}^{e} & =\int_{\Omega_{e}} \mathbf{B}_{\mathbf{u}}^{T} \mathbf{C}^{m} \mathbf{B}_{\mathbf{u}} d V, \\
\mathbf{K}_{\mathbf{u} \theta}^{e} & =-\int_{\Omega_{e}} \mathbf{B}_{\mathbf{u}}^{T} \boldsymbol{\alpha}^{m} \mathbf{N}_{\theta} d V, \\
\mathbf{K}_{\mathbf{u} \eta}^{e} & =-\int_{\Omega_{e}} \mathbf{B}_{\mathbf{u}}^{T} \boldsymbol{\beta}^{m} \mathbf{N}_{\eta} d V, \\
\mathbf{K}_{\theta \theta}^{e} & =\int_{\Omega_{e}} \mathbf{B}_{\theta}^{T} \mathbf{K}^{m} \mathbf{B}_{\theta} d V, \\
\mathbf{K}_{\eta \eta}^{e} & =\int_{\Omega_{e}} \mathbf{B}_{\eta}^{T} \mathbf{D}^{m} \mathbf{B}_{\eta} d V .
\end{aligned}
$$

Analogously, damping matrices relative to each element read

$$
\begin{aligned}
& \mathbf{C}_{\theta \theta}^{e}=\int_{\Omega_{e}} \mathbf{N}_{\theta}^{T} p^{m} \mathbf{N}_{\theta} d V, \\
& \mathbf{C}_{\eta \eta}^{e}=\int_{\Omega_{e}} \mathbf{N}_{\eta}^{T} q^{m} \mathbf{N}_{\eta} d V, \\
& \mathbf{C}_{\theta \mathbf{u}}^{e}=\int_{\Omega_{e}} \mathbf{N}_{\theta}^{T} \boldsymbol{\alpha}^{m} \mathbf{B}_{\mathbf{u}} d V, \\
& \mathbf{C}_{\theta \mathbf{u}}^{e}=\int_{\Omega_{e}} \mathbf{N}_{\theta}^{T} \psi^{m} \mathbf{N}_{\eta} d V, \\
& \mathbf{C}_{\eta \mathbf{u}}^{e}=\int_{\Omega_{e}} \mathbf{N}_{\eta}^{T} \boldsymbol{\beta}^{m} \mathbf{B}_{\mathbf{u}} d V, \\
& \mathbf{C}_{\eta \theta}^{e}=\int_{\Omega_{e}} \mathbf{N}_{\eta}^{T} \psi^{m} \mathbf{N}_{\theta} d V,
\end{aligned}
$$

and the elemental mass matrix has the form

$$
\mathbf{M}_{\mathbf{u u}}^{e}=\int_{\Omega_{e}} \mathbf{N}_{\mathbf{u}}^{T} \rho^{m} \mathbf{N}_{\mathbf{u}} d V .
$$

The elemental external force vectors have the following expressions

$$
\begin{aligned}
\mathbf{f}_{\mathbf{u}}^{e} & =\int_{\Omega_{e}} \mathbf{N}_{\mathbf{u}}^{T} \mathbf{b} d V+\int_{\partial \Omega_{e_{\boldsymbol{\sigma}}}} \mathbf{N}_{\mathbf{u}}^{t} \overline{\mathbf{t}} d S, \\
\mathbf{f}_{\theta}^{e} & =\int_{\Omega_{e}} \mathbf{N}_{\theta}^{T} r d V-\int_{\partial \Omega_{\Omega_{\mathbf{q}}}} \mathbf{N}_{\theta}^{T} \bar{q} d S, \\
\mathbf{f}_{\eta}^{e} & =\int_{\Omega_{e}} \mathbf{N}_{\eta}^{T} s d V-\int_{\partial \Omega_{\Omega_{\mathbf{j}}}} \mathbf{N}_{\eta}^{t} \bar{j} d S .
\end{aligned}
$$

Equations (148)-(149, therefore, can be written in the following form, after assembling elemental contributions 150)-(153) into the relative global ones

$$
\begin{aligned}
& \mathbf{K}_{\mathbf{u u}} \mathbf{u}+\mathbf{K}_{\mathbf{u} \theta} \boldsymbol{\theta}+\mathbf{K}_{\mathbf{u} \eta} \boldsymbol{\eta}=\mathbf{f}_{\mathbf{u}}-\mathbf{M}_{\mathbf{u u}} \ddot{\mathbf{u}} \\
& \mathbf{K}_{\theta \theta} \boldsymbol{\theta}+\mathbf{C}_{\theta \mathbf{u}} \dot{\mathbf{u}}+\mathbf{C}_{\theta \eta} \dot{\boldsymbol{\eta}}=\mathbf{f}_{\theta}-\mathbf{C}_{\theta \theta} \dot{\boldsymbol{\theta}} \\
& \mathbf{K}_{\eta \eta} \boldsymbol{\eta}+\mathbf{C}_{\eta \mathbf{u}} \dot{\mathbf{u}}+\mathbf{C}_{\eta \theta} \dot{\boldsymbol{\theta}}=\mathbf{f}_{\eta}-\mathbf{C}_{\eta \eta} \dot{\boldsymbol{\eta}} .
\end{aligned}
$$

After performing bilateral Laplace transform (82) on system (154), taking into account derivation rule (83), one obtains the following system expressed in terms of vector $\hat{\mathbf{z}}=(\hat{\mathbf{u}} \hat{\boldsymbol{\theta}} \hat{\boldsymbol{\eta}})^{T}$ containing the microfields in the Laplace domain

$$
\left(\mathbf{K}_{\mathbf{u u}}+\omega^{2} \mathbf{M}_{\mathbf{u u}}\right) \hat{\mathbf{u}}+\mathbf{K}_{\mathbf{u} \theta} \hat{\boldsymbol{\theta}}+\mathbf{K}_{\mathbf{u} \eta} \hat{\boldsymbol{\eta}}=\hat{\mathbf{f}}_{\mathbf{u}}
$$




$$
\begin{aligned}
& \left(\mathbf{K}_{\theta \theta}+\omega \mathbf{C}_{\theta \theta}\right) \hat{\boldsymbol{\theta}}+\omega \mathbf{C}_{\theta \mathbf{u}} \hat{\mathbf{u}}+\omega \mathbf{C}_{\theta \eta} \hat{\boldsymbol{\eta}}=\hat{\mathbf{f}}_{\theta}, \\
& \left(\mathbf{K}_{\eta \eta}+\omega \mathbf{C}_{\eta \eta}\right) \hat{\boldsymbol{\eta}}+\omega \mathbf{C}_{\eta \mathbf{u}} \hat{\mathbf{u}}+\omega \mathbf{C}_{\eta \theta} \hat{\boldsymbol{\theta}}=\hat{\mathbf{f}}_{\eta} .
\end{aligned}
$$

Exploiting the periodicity of the medium, generalized Christoffel equations 155 can be studied in the periodic cell $\mathcal{A}$. By virtue of Bloch's theorem, Floquet-Bloch boundary conditions have to be applied to elementary cell $\mathcal{A}$ in order to obtain its dispersion relations. Following the procedure described in (Langley, 1993, Phani et al. 2006), degrees of freedom contained in vector $\hat{\mathbf{z}}$ can be reorganized as $\hat{\mathbf{z}}=$ $\left(\hat{\mathbf{z}}_{\ell} \hat{\mathbf{z}}_{r} \hat{\mathbf{z}}_{b} \hat{\mathbf{z}}_{t} \hat{\mathbf{z}}_{\ell b} \hat{\mathbf{z}}_{r b} \hat{\mathbf{z}}_{\ell t} \hat{\mathbf{z}}_{r t} \hat{\mathbf{z}}_{i}\right)^{T}$, where subscripts $\ell, r, b, t$, and $i$ denote, respectively, the left, right, bottom, top, and internal nodes of a generic cell and double subscripts indicate corner nodes. Floquet-Bloch boundary conditions are written as

$$
\begin{array}{ll}
\hat{\mathbf{z}}_{r}=e^{i k_{1} d_{1}} \hat{\mathbf{z}}_{\ell}, & \hat{\mathbf{f}}_{r}=-e^{i k_{1} d_{1}} \hat{\mathbf{f}}_{\ell}, \\
\hat{\mathbf{z}}_{t}=e^{i k_{2} d_{2}} \hat{\mathbf{z}}_{b}, & \hat{\mathbf{f}}_{t}=-e^{i k_{2} d_{2}} \hat{\mathbf{f}}_{b}, \\
\hat{\mathbf{z}}_{r b}=e^{i k_{1} d_{1}} \hat{\mathbf{z}}_{\ell b}, & \hat{\mathbf{f}}_{r b}=-e^{i k_{1} d_{1}} \hat{\mathbf{f}}_{\ell b}, \\
\hat{\mathbf{z}}_{\ell t}=e^{i k_{2} d_{2}} \hat{\mathbf{z}}_{\ell b}, & \hat{\mathbf{f}}_{\ell t}=-e^{i k_{2} d_{2}} \hat{\mathbf{f}}_{\ell b}, \\
\hat{\mathbf{z}}_{r t}=e^{i\left(k_{1} d_{1}+k_{2} d_{2}\right)} \hat{\mathbf{z}}_{\ell b}, & \hat{\mathbf{f}}_{r t}=-e^{i\left(k_{1} d_{1}+k_{2} d_{2}\right)} \hat{\mathbf{f}}_{\ell b} .
\end{array}
$$

where $i$ is the imaginary unit s.t. $i^{2}=-1$ and $\mathbf{k}=k_{1} \mathbf{e}_{1}+k_{2} \mathbf{e}_{2} \in \mathcal{B}$ is the wave vector with wave numbers $k_{1}$ and $k_{2}$, and $\mathcal{B}=\left[-\pi / d_{1}, \pi / d_{1}\right] \times\left[-\pi / d_{2}, \pi / d_{2}\right]$ is the first Brillouin zone of cell $\mathcal{A}$ having orthogonal periodicity vectors $\mathbf{v}_{1}=d_{1} \mathbf{e}_{1}$ and $\mathbf{v}_{\mathbf{2}}=d_{2} \mathbf{e}_{2}$. Boundary conditions 156 allow to define the following transformation

$$
\hat{\mathbf{z}}=\mathbf{T} \hat{\mathbf{q}}
$$

with matrix $\mathbf{T}$ defined as

$$
\mathbf{T}=\left(\begin{array}{cccc}
\mathbf{I} & \mathbf{0} & \mathbf{0} & \mathbf{0} \\
\mathbf{I} e^{i k_{1} d_{1}} & \mathbf{0} & \mathbf{0} & \mathbf{0} \\
\mathbf{0} & \mathbf{I} & \mathbf{0} & \mathbf{0} \\
\mathbf{0} & \mathbf{I} e^{i k_{2} d_{2}} & \mathbf{0} & \mathbf{0} \\
\mathbf{0} & \mathbf{0} & \mathbf{I} & \mathbf{0} \\
\mathbf{0} & \mathbf{0} & \mathbf{I} e^{i k_{1} d_{1}} & \mathbf{0} \\
\mathbf{0} & \mathbf{0} & \mathbf{I} e^{i k_{2} d_{2}} & \mathbf{0} \\
\mathbf{0} & \mathbf{0} & \mathbf{I} e^{i\left(k_{1} d_{1}+k_{2} d_{2}\right)} & \mathbf{0}
\end{array}\right)
$$

and vector $\hat{\mathbf{q}}$ of reduced independent degrees of freedom expressed in the form

$$
\hat{\mathbf{q}}=\left(\begin{array}{c}
\hat{\mathbf{z}}_{\ell} \\
\hat{\mathbf{z}}_{b} \\
\hat{\mathbf{z}}_{\ell b} \\
\mathbf{z}_{i}
\end{array}\right) .
$$

Substitution of equation 157 into governing equations of motion 155 and premultiplication by the Hermitian transpose of $\mathbf{T}$, named $\mathbf{T}^{H}$, in order to enforce equilibrium, lead to

$$
\left(\omega^{2} \mathbf{T}^{H} \mathbf{M} \mathbf{T}+\omega \mathbf{T}^{H} \mathbf{C T}+\mathbf{T}^{H} \mathbf{K} \mathbf{T}\right) \hat{\mathbf{q}}=\mathbf{T}^{H} \hat{\mathbf{f}}
$$

where $\mathbf{M}, \mathbf{C}$, and $\mathbf{K}$ represent, respectively, the global mass, damping, and stiffness matrices. In the case of free wave motion $(\hat{\mathbf{f}}=0)$ it results $\mathbf{T}^{H} \hat{\mathbf{f}}=\mathbf{0}$, and equation 160 defines a quadratic generalized eigenvalue problem whose solution, for each value of wave vector $\mathbf{k} \in \mathbb{R}^{2}$, gives the complex frequency $\omega$ as the generalized eigenvalue and $\hat{\mathbf{q}}$ as the generalized eigenvector. Real and imaginary parts of the complex angular frequency $\omega=\omega_{r}+i \omega_{i}$, characterize the damping and the propagation mode, respectively, of dispersive Bloch waves propagating inside the heterogeneous material. Finally, quadratic eigenvalue problem 160 can be tranformed into an equivalent linear one in the following way

$$
\left(\omega\left(\begin{array}{cc}
\mathbf{T}^{H} \mathbf{M T} & \mathbf{0} \\
\mathbf{0} & \mathbf{I}
\end{array}\right)+\left(\begin{array}{cc}
\mathbf{T}^{H} \mathbf{C T} & \mathbf{T}^{H} \mathbf{K T} \\
-\mathbf{I} & \mathbf{I}
\end{array}\right)\right)\left(\begin{array}{c}
\omega \hat{\mathbf{q}} \\
\hat{\mathbf{q}}
\end{array}\right)=\left(\begin{array}{l}
\mathbf{0} \\
\mathbf{0}
\end{array}\right)
$$

which admits a non trivial solution $(\omega \hat{\mathbf{q}} \hat{\mathbf{q}})^{T}$ only if the linear operator mutiplying the generalized eigenvector is not invertible. 